\title{
Statistical understanding of quark and lepton masses in Gaussian landscapes
}

\author{
Lawrence J. Hall, ${ }^{1}$ Michael P. Salem, ${ }^{2}$ and Taizan Watari ${ }^{3}$ \\ ${ }^{1}$ Department of Physics and Lawrence Berkeley National Laboratory, University of California, Berkeley, California 94720, USA \\ ${ }^{2}$ California Institute of Technology, Pasadena, California 91125, USA \\ ${ }^{3}$ Department of Physics, The University of Tokyo, Tokyo, 113-0033, Japan
}

(Received 9 August 2007; published 5 November 2007)

\begin{abstract}
The fundamental theory of nature may allow a large landscape of vacua. Even if the theory contains a unified gauge symmetry, the 22 flavor parameters of the standard model, including neutrino masses, may be largely determined by the statistics of this landscape, and not by any symmetry. Then the measured values of the flavor parameters do not lead to any fundamental symmetries, but are statistical accidents; their precise values do not provide any insights into the fundamental theory, rather the overall pattern of flavor reflects the underlying landscape. We investigate whether random selection from the statistics of a simple landscape can explain the broad patterns of quark, charged lepton, and neutrino masses and mixings. We propose Gaussian landscapes as simplified models of landscapes where Yukawa couplings result from overlap integrals of zero-mode wave functions in higher-dimensional supersymmetric gauge theories. In terms of just five free parameters, such landscapes can account for all gross features of flavor, including the hierarchy of quark and charged-lepton masses; small quark mixing angles in the basis with quarks arranged according to mass, with 13 mixing less than 12 and 23 mixing; very light Majorana neutrino masses, with the solar to atmospheric neutrino mass ratio consistent with data; distributions for leptonic mixings $\sin 2 \theta_{12}$ and $\sin 2 \theta_{23}$ that are peaked at large values, while the distribution for $\sin 2 \theta_{13}$ is peaked at low values; and order unity $C P$-violating phases in both the quark and lepton sectors. While the statistical distributions for flavor parameters are broad, the distributions are robust to changes in the geometry of the extra dimensions. Constraining the distributions by loose cuts about observed values leads to narrower distributions for neutrino measurements of $\theta_{13}, C P$ violation, and neutrinoless double beta decay.
\end{abstract}

DOI: 10.1103/PhysRevD.76.093001

PACS numbers: 12.15.Ff, 11.25.Mj, 14.60.Pq

\section{INTRODUCTION}

The standard model $^{1}$ describes all laboratory data with 28 free parameters. Of these, 22 arise from the Yukawa matrices $\lambda^{u, d, e}$ and the coupling matrix $C$ that appear in the flavor interactions

$$
\mathcal{L}_{\text {flav }}=\lambda_{i j}^{u} \bar{u}_{i} q_{j} h+\lambda_{k j}^{d} \bar{d}_{k} q_{j} h^{*}+\lambda_{a i}^{e} \bar{e}_{a} l_{i} h^{*}+\frac{C_{i j}}{M} l_{i} l_{j} h h,
$$

where $q, l(\bar{u}, \bar{d}, \bar{e})$ are the left (right) handed quark and lepton fields. Of these 22 flavor parameters, 17 are measured, with varying levels of accuracy. The remaining five parameters are all in the neutrino sector and two of these have upper limits. Given several decades of continued progress on improving the accuracy of the experimental measurements, the most striking fact is that there is nothing approaching a standard theory of the origin of these parameters. Not only are we ignorant about the overall picture of flavor, we do not have a convincing explanation for the value of any of the 17 measured parameters.

A complete theory of flavor would provide answers to three very different questions:

(i) What is the origin of the fermion quantum numbers and why are there three generations?

\footnotetext{
${ }^{1}$ We everywhere refer to the "standard model" as the theory including the dimension-five operators of (1).
}

(ii) What determines the qualitative pattern of the quark and lepton mass matrices? For example, why do the charged fermion masses and mixings have a hierarchical pattern, while in the neutrino sector there are large mixing angles?

(iii) What determines the precise values of the 22 flavor parameters?

The couplings $\lambda^{u, d, e}$ and $C$ are symmetry-breaking parameters of the flavor-symmetry group $U(3)^{5}$, where one $U(3)$ factor acts on each of $q, l, \bar{u}, \bar{d}, \bar{e}$. The dominant approach to constructing theories of flavor is to use symmetries to reduce the number of free parameters, $n$. An underlying flavor group $G_{f} \subset U(3)^{5}$ and a more unified gauge symmetry both limit $n$, leading to precise predictions if $n<n_{\mathrm{obs}}$, the number of observables. A hierarchy of symmetry-breaking scales can lead to small dimensionless parameters that explain qualitative features of the mass matrices [1], and there are many realizations with $G_{f}$ both Abelian and non-Abelian. Still, it is striking that the progress along these lines is limited, even as more precise data have become available. Perhaps this is a sign that a completely new approach is needed.

The cosmological dark energy [2] apparently has little to do with flavor. However, it may be the first evidence for a huge landscape of vacua, with the observed value of the cosmological constant resulting from environmental selection for large scale structure [3]. If we take string theory to be a theory of a landscape rather than a theory of a single 
vacuum, then what are the implications for flavor? The enormous number of vacua, required for a sufficiently fine scan of the cosmological constant, results from the large number of ways background fluxes can be arranged on nontrivial compact manifolds of extra spatial dimensions [4]. The cosmological constant, however, is not the only parameter that scans over such a landscape. The Yukawa couplings may also vary from one universe to another.

In any landscape of vacua, the Yukawa couplings have a relevant dependence on $n=n_{S}+n_{F}$ parameters, where $n_{S}$ of the parameters scan and $n_{F}$ of them are fixed. If $n<$ $n_{\text {obs }}$ then data can determine the subset of vacua in which we happen to live and $n_{\text {obs }}-n$ predictions can be made. A certain simple model with a single extra dimension has $n_{\mathrm{obs}}-n=1$, giving a single prediction [5]. If $n>n_{\mathrm{obs}}$, however, then no such precise predictions are possible. Some flavor parameters - such as the electron, up and down masses - may be determined by environmental effects [6]. The top mass may also be determined along this line [7], while selecting for leptogenesis may be a key to understanding some parameters in the lepton sector. However, most of the flavor parameters do not seem to be strongly selected. Therefore it could be that most flavor parameters have values that simply reflect some underlying probability distribution over an enormous number of solutions to the fundamental theory. The precise value of any such observable is accidental and not fundamental, since any other nearby value would be just as probable. Although it may be unappealing that there is nothing fundamental or beautiful relating the flavor parameters in our universe, so far this possibility cannot be dismissed, especially given that no such relations have been found.

The large mixing angles observed in atmospheric and solar neutrino oscillations inspired the idea that the relevant Yukawa couplings of the neutrino sector are governed by randomness rather than by flavor symmetries [8]. By introducing a simple probability distribution for the elements in the Dirac and Majorana neutrino mass matrices, probability distributions for the neutrino observables were generated and found to agree well with data. However, a complete landscape approach to the theory of flavor must explain many other things; there are rich structures among the observed flavor parameters. Reference [9] took a bold step in this direction, studying masses and mixings in both the quark and lepton sectors by introducing a simple probability distribution for the Yukawa couplings. We begin in Sec. II by analyzing the toy landscape introduced in [9]. While the main purpose of this section is to clarify how hierarchy among quark masses is generated in this model and to point out its limitations, we also introduce an approximate analytical study of the probability distribution of flavor observables. This turns out to be a useful warm-up exercise for the subsequent sections.

Ideally, a statistical approach to the theory of flavor would be both phenomenologically successful and theoretically well motivated. However, to determine the proba- bility distributions for flavor parameters from a purely topdown calculation is not an easy task; meanwhile the number of observables is limited, and such low-energy information does not constrain the underlying probability distributions in a purely bottom-up approach. We consider as a practical strategy to use string theory as a guide in deducing the form of the probability distributions, while using known experimental constraints to try to find a phenomenologically successful model within this restricted set of possibilities. In a given compactification of string theory, the Yukawa couplings are determined by an overlap integration over the extra spatial dimensions. Scanning moduli parameters of the gauge-field configuration on the extra dimensions corresponds to scanning zeromode wave functions, and hence this corresponds to scanning Yukawa couplings. If the wave functions of the quarks, leptons, and the Higgs are peaked at different locations, then the overlap integral can lead to small Yukawa matrix elements. Hence localization in extra dimensions is an alternative to symmetries for generating fermion mass hierarchies [10].

In Secs. III through VI we introduce simple "Gaussian landscapes" and study the resulting distributions for quark and lepton masses and mixings. ${ }^{2}$ These landscapes have features expected from certain string landscapes, for which they can be viewed as simplified or toy models. The key feature of a Gaussian landscape is that all quark, lepton, and Higgs fields have zero-mode wave functions with Gaussian profiles in the extra dimensions, and that the centers of these profiles all scan independently with flat probability distributions over the volume of the extra dimensions. For simplicity, the number of free parameters used to describe the geometry of the extra dimensions and the widths of the Gaussian profiles is kept to a minimum. We find the observed quark and lepton masses and mixings can be typical in such simple landscapes.

In Sec. III we introduce a Gaussian landscape for quarks on a circle $S^{1}$, with all Gaussian profiles having the same width. Numerical probability distributions for the nine $C P$-conserving flavor observables are provided, and a qualitative semianalytic description of these distributions is derived. The results are compared with those that result from introducing approximate flavor symmetries, with some similarities and some differences emerging. Finally, the effects of possible environmental selection on the top quark mass is studied. The large number of flavor parameters in the standard model allows for a reasonably significant evaluation of goodness-of-fit between a Gaussian landscape and the observed flavor structure. This is described in Sec. IV, with the $S^{1}$ Gaussian landscape used for illustration. The effects on the quark sector from adding more dimensions to the Gaussian landscape are examined

\footnotetext{
${ }^{2} \mathrm{~A}$ brief summary of some of the major results of this work can be found in [11].
} 
in Sec. V, together with a preliminary study of the effects of geometry.

In Sec. VI the Gaussian landscape is extended to include the lepton sector. Motivated by the expectation of supersymmetry in the higher-dimensional theory, Yukawa couplings for both Dirac and Majorana neutrino masses are introduced in the Gaussian landscape, with light neutrinos resulting from the usual seesaw mechanism in four dimensions. Larger mixing angles in the lepton sector might arise from assigning the appropriate fermions larger Gaussian widths, but these are found to largely cancel between charged and neutral sectors. However, this cancellation is prevented by introducing $C P$-violating phases in the Gaussian profiles. This suggests a connection in Gaussian landscapes between large $C P$ violation and large leptonic mixing angles. In addition to providing numerical distributions for the observed lepton flavor parameters, probability distributions are predicted for the leptonic mixing angle $\theta_{13}$, the $C P$ phase in neutrino oscillations, and the Majorana mass relevant for neutrinoless double beta decay, illustrating how these toy models for the string landscape can connect to future experimental data. Finally, the extent to which these Gaussian landscapes arise from unified supersymmetric field theories in higher dimensions is examined in Sec. VII. Such theories have huge symmetries ultimately all entries of the Yukawa matrices arise from a unified supersymmetric gauge coupling - so that the complicated pattern of observed masses and mixings can arise from a very simple mechanism: the scanning of the centers of the Gaussian profiles.

\section{PRELUDE: HIERARCHY WITHOUT FLAVOR SYMMETRY}

In previous work it has been suggested that the components of the Yukawa matrices $\lambda^{u, d, e}$ and the coupling matrix $C$ are selected randomly and independently of each other [8,9]. For example, in neutrino anarchy [8] one finds that the large mixing angles underlying neutrino oscillation are typical of the lepton interactions that arise when each element of the matrices $\lambda^{e}$ and $C$ is independently selected from the simple probability distributions

$$
\frac{d P\left(\lambda^{e}\right)}{d \lambda^{e}}=\text { const, } \quad \frac{d P(C)}{d C}=\text { const, }
$$

or from distributions such as

$$
\begin{aligned}
& \frac{d P\left(\lambda^{e}\right)}{d \ln \lambda^{e}}=\mathrm{const} \quad \text { for } \lambda_{\min }^{e}<\lambda^{e}<\lambda_{\max }^{e}, \\
& \frac{d P(C)}{d \ln C}=\mathrm{const} \quad \text { for } C_{\min }<C<C_{\max },
\end{aligned}
$$

where $\lambda_{\min \text { max }}^{e}$ and $C_{\min \text { max }}$ are of order unity (for more details see [8,12]). Such an absolute anarchy of lepton couplings tends to result in comparable mass eigenvalues. On the other hand, [9] introduced a power-law probability distribution for the Yukawa matrix elements, $d P(\lambda) / d \lambda \propto$ $\lambda^{-\delta}$ for $\lambda_{\min }<\lambda<\lambda_{\max }$ and $d P(\lambda) / d \lambda=0$ otherwise. By assuming $\lambda_{\min } \ll \lambda_{\max }$ and choosing $\delta$ appropriately, quark Yukawa matrices with each matrix element following such a distribution can roughly accommodate the hierarchical pattern of quark mass eigenvalues [9]. According to [9], $\delta=1.16$ provides the best fit to the quark sector.

In this paper we propose a significant modification to these ideas. However, as a prelude to discussing our proposal it is worthwhile to first study the model of [9] in greater detail. To simplify the analysis we specialize to the particular case $\delta=1$, such that

$$
\frac{d P(\lambda)}{d \log _{10} \lambda}= \begin{cases}0 & \text { for } \lambda>\lambda_{\max } \\ 1 / \log _{10}\left(\lambda_{\max } / \lambda_{\min }\right) & \text { for } \lambda_{\min }<\lambda<\lambda_{\max } \\ 0 & \text { for } \lambda<\lambda_{\min }\end{cases}
$$

Henceforth we refer to this distribution as a scale-invariant distribution. In this section we also restrict attention to the quark sector. Note that $C P$-violating phases are not introduced in this landscape because all of the matrix elements are real-valued. A pair of $3 \times 3$ Yukawa matrices $\lambda^{u}$ and $\lambda^{d}$ is generated by choosing each of the 18 matrix elements randomly according to the distribution (4). The Yukawa matrices are then diagonalized using separate left- and right-handed unitary transformations, and the quark masses and mixings are calculated from the eigenvalues of the diagonalized Yukawa matrices and the resulting Cabibbo-Kobayashi-Maskawa (CKM) matrix. The above process is then repeated to generate an ensemble of these nine observables.

\section{A. The distribution of mass eigenvalues}

We first study the distributions of the quark masses. Since the up- and down-type Yukawa matrices are generated independently of each other and in exactly the same way, the distributions of masses for these sectors are exactly the same. Thus we only need to study one of the two sectors. Results of a numerical study are shown in Fig. 1, where we have chosen $\lambda_{\min } / \lambda_{\max }=10^{-9.1}$. With some approximations we can understand the shapes of these distributions. Let $\lambda_{3}^{\prime}$ denote the largest element of the $3 \times$ 3 matrix $\lambda$. Meanwhile, the largest element of the $2 \times 2$ submatrix of $\lambda$ that excludes $\lambda_{3}^{\prime}$ is denoted $\lambda_{2}^{\prime}$. For example, in the matrix

$$
\lambda=\left(\begin{array}{c|cc}
\lambda_{23} & \lambda_{2}^{\prime} & \lambda_{21} \\
\lambda_{13} & \lambda_{12} & \lambda_{1}^{\prime} \\
\hline \lambda_{3}^{\prime} & \lambda_{32} & \lambda_{31}
\end{array}\right),
$$

$\lambda_{3}^{\prime}$ is the largest among the nine entries and $\lambda_{2}^{\prime}$ the largest in the upper right $2 \times 2$ submatrix. Given this characterization, the probability distribution for the variables $\lambda_{1,2,3}^{\prime}$ and 

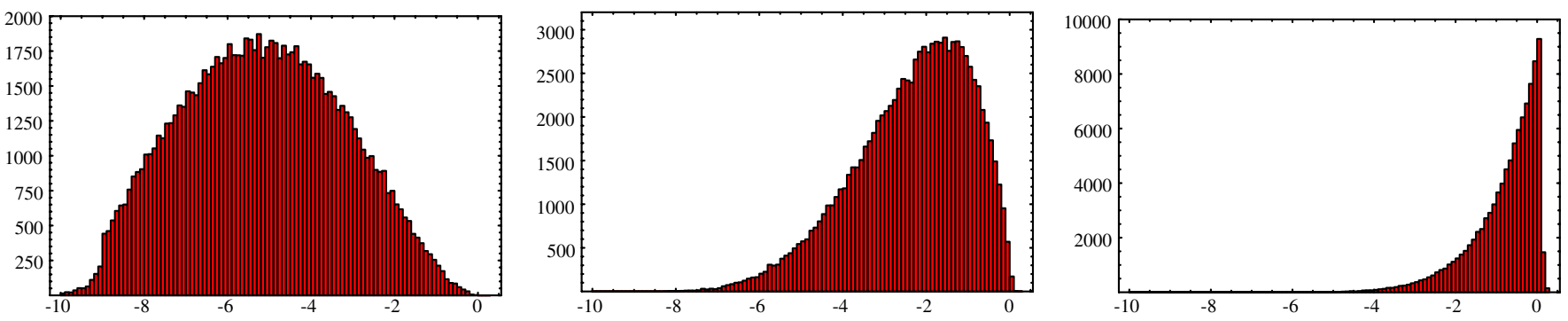

FIG. 1 (color online). Distributions of the three eigenvalues of Yukawa matrices whose each element follows the distribution (4). From left to right the three panels correspond to the smallest, middle, and largest eigenvalues. The sum of all three distributions reproduces Fig. 9a of [9]. We used $\log _{10} \lambda_{\min }=-9$ and $\log _{10} \lambda_{\max }=0.1$ for this simulation.

$\lambda_{i j}$ is

$$
\begin{aligned}
d P\left(x_{1,2,3}^{\prime}, x_{i j}\right)= & 36 \Theta\left(x_{3}^{\prime}-x_{2}^{\prime}\right) \Theta\left(x_{3}^{\prime}-x_{32}\right) \Theta\left(x_{3}^{\prime}-x_{23}\right) \\
& \times \Theta\left(x_{3}^{\prime}-x_{31}\right) \Theta\left(x_{3}^{\prime}-x_{13}\right) \Theta\left(x_{2}^{\prime}-x_{1}^{\prime}\right) \\
& \times \Theta\left(x_{2}^{\prime}-x_{21}\right) \Theta\left(x_{2}^{\prime}-x_{12}\right) d x_{1}^{\prime} d x_{2}^{\prime} d x_{3}^{\prime} d x_{12} \\
& \times d x_{21} d x_{13} d x_{31} d x_{23} d x_{32},
\end{aligned}
$$

where $\Theta$ is the step function. The factor of 36 comes from the nine possible locations for $\lambda_{3}^{\prime}$ times the four possible locations for $\lambda_{2}^{\prime}$. In addition we have introduced the notation

$$
x_{i}^{\prime} \equiv \frac{\ln \left(\lambda_{i}^{\prime} / \lambda_{\min }\right)}{\ln \left(\lambda_{\max } / \lambda_{\min }\right)}, \quad x_{i j} \equiv \frac{\ln \left(\lambda_{i j} / \lambda_{\min }\right)}{\ln \left(\lambda_{\max } / \lambda_{\min }\right)} .
$$

The largest mass eigenvalue of (5) is approximately $\lambda_{3}^{\prime}$; this approximation is poor if one of $\lambda_{32}, \lambda_{31}, \lambda_{23}$, and $\lambda_{13}$ is almost as large $\lambda_{3}^{\prime}$, but this is unlikely if $\ln \left(\lambda_{\max } / \lambda_{\min }\right)$ is large. We call this largest eigenvalue $\lambda_{3}$ and define $x_{3}$ analogously to $x_{3}^{\prime}$. The probability distribution of $x_{3}$ is therefore approximated by integrating out from (6) all the variables except $x_{3}^{\prime}$ :

$$
d P\left(x_{3}\right) \simeq 9 x_{3}^{8} d x_{3} .
$$

Meanwhile, we approximate the middle eigenvalue $\lambda_{2}$ by $\lambda_{2}^{\prime}$ and the smallest eigenvalue $\lambda_{1}$ by $\lambda_{1}^{\prime}$. This approximation is poor when the seesaw contributions $\left(\lambda_{i 3} \lambda_{3 j}\right) / \lambda_{3}^{\prime}$ and $\left(\lambda_{12} \lambda_{21}\right) / \lambda_{2}^{\prime}$ are larger than $\lambda_{2}^{\prime}$ and $\lambda_{1}^{\prime}$, respectively. Thus we do not expect this approximation to be reliable for small values of $x_{2}$ and $x_{1}$. Nevertheless, integrating out all of other variables we find

$$
\begin{gathered}
d P\left(x_{2}\right)=\frac{36}{5} x_{2}^{3}\left(1-x_{2}^{5}\right) d x_{2}, \\
d P\left(x_{1}\right)=\frac{36}{5}\left(\frac{1-x_{1}^{3}}{3}-\frac{1-x_{1}^{8}}{8}\right) d x_{1} .
\end{gathered}
$$

The probability distributions (8)-(10) are shown in Fig. 2. Remarkably, they capture the gross features of the numerical results in Fig. 1. Therefore we use these distributions to examine the qualitative aspects of the mass distributions that follow from the landscape (4).

The average $\left\langle x_{i}\right\rangle$ and the standard deviation $\sigma_{i}$ of the three Yukawa eigenvalues (both on a logarithmic scale) can be calculated from the distributions (8)-(10):

$$
\begin{aligned}
\left\langle x_{3}\right\rangle=0.90, & \sigma_{3}=0.09, & x_{3} \sim[0.81-0.99], \\
\left\langle x_{2}\right\rangle=0.72, & \sigma_{2}=0.16, & x_{2} \sim[0.56-0.88], \\
\left\langle x_{1}\right\rangle=0.36, & \sigma_{1}=0.22, & x_{1} \sim[0.14-0.58] .
\end{aligned}
$$
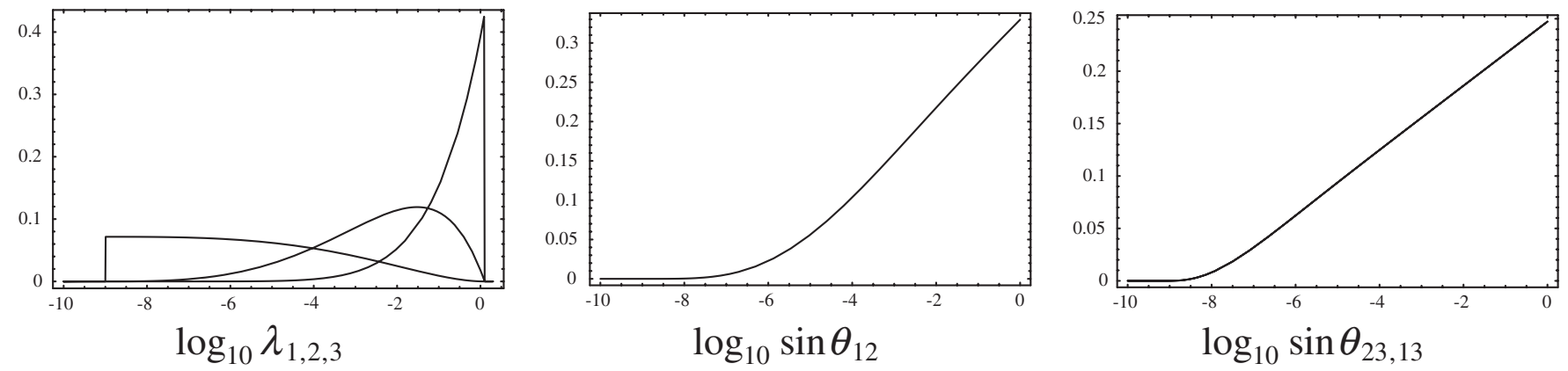

FIG. 2. The approximate distributions of the three eigenvalues given in (8)-(10), and those of mixing angles given in (15) and (16). We have used the same $\lambda_{\max }$ and $\lambda_{\min }$ as are used in Fig. 1. 
The three eigenvalues are on average well separated and they overlap with neighboring eigenvalues only slightly at 1 standard deviation. Even this slight overlap between the distributions is misleading. Recall that by definition for any particular set of Yukawa matrices we have $x_{3}^{\prime}>x_{2}^{\prime}>x_{1}^{\prime}$. Thus the combined distribution for the eigenvalues is not a naive product of (8)-(10) but is given by integrating the other six variables out of (6). This gives

$$
d P\left(x_{1}, x_{2}, x_{3}\right) \simeq 36 x_{3}^{4} x_{2}^{2} \Theta\left(x_{3}-x_{2}\right) \Theta\left(x_{2}-x_{1}\right) d x_{1} d x_{2} d x_{3} .
$$

Thus it happens that in the subset of cases where $x_{3}$ is small, the distribution of $x_{2}$ is pushed to even smaller values. Hence the three eigenvalues tend to be well separated even in the logarithmic scale, and only rarely are adjacent eigenvalues comparable. Note that none of this depends on the choice of $\lambda_{\min }$ and $\lambda_{\max }$. In short, hierarchical structure (Yukawa eigenvalues well separated in logarithmic scale) is generated statistically in a landscape where each matrix element independently follows the scale-invariant distribution (4). Whether the hierarchy is large or small is determined by whether $\log _{10}\left(\lambda_{\max } / \lambda_{\min }\right)$ is large or small.

\section{B. Pairing structure in electroweak interactions}

Let us now study the mixing angles. Figure 3 shows the distributions of mixing angles in the quark sector that result from a numerical simulation where each element of both the up-type and down-type Yukawa matrices is assumed to follow the distribution (4) independently. ${ }^{3}$ The probability distribution functions of the mixing angles are shown against the axes of $d \theta_{12}, d \theta_{23}$, and $d\left(\sin \theta_{13}\right)$ because the invariant measure ${ }^{4}$ of $\mathrm{SO}(3)$ mixing matrices is $d \theta_{12} \wedge$ $d \theta_{23} \wedge d\left(\sin \theta_{13}\right)$. Since in this model the Yukawa couplings are all real valued and positive valued, the CKM quark mixing matrices are $\mathrm{SO}(3)$ matrices.

The prominent feature of these distributions is the twin peaks at $\theta_{i j}=0$ and $\theta_{i j}=\pi / 2$ for all three mixing angles. This feature is straightforward to understand. Suppose that

${ }^{3}$ Using (8)-(10) we find these distribution functions to be given by the approximate analytic form:

$$
\begin{aligned}
& d P(t) \simeq \frac{3}{50}\left(10-15 t+6 t^{4}-t^{9}\right)\left(5-8 t^{3}+3 t^{8}\right) d t, \\
& \text { where } t \equiv \frac{\ln \sin \theta_{12}}{\ln \left(\lambda_{\min } / \lambda_{\max }\right)},
\end{aligned}
$$

$d P(t) \simeq \frac{9}{32}\left(8-9 t+t^{9}\right)\left(1-t^{8}\right) d t, \quad$ where $t \equiv \frac{\ln \sin \theta_{23,13}}{\ln \left(\lambda_{\min } / \lambda_{\max }\right)}$

\footnotetext{
${ }^{4}$ The importance of the invariant measure is emphasized in [12].
}

the randomly generated Yukawa matrices are of the form

$$
\lambda_{i j}^{u} \sim\left(\begin{array}{ccc}
* & \lambda_{2}^{\prime u} & \bullet \\
* & \bullet & \lambda_{1}^{\prime u} \\
\lambda_{3}^{\prime u} & * & *
\end{array}\right), \quad \lambda_{k j}^{d} \sim\left(\begin{array}{ccc}
\bullet & * & \lambda_{2}^{\prime d} \\
* & \lambda_{3}^{\prime d} & * \\
\lambda_{1}^{\prime d} & * & \bullet
\end{array}\right)
$$

where the *'s are assumed to be less than $\lambda_{3}^{\prime \prime}$ or $\lambda_{3}^{\prime d}$, and the -'s less than $\lambda_{2}^{\prime \prime}$ or $\lambda_{2}^{\prime d}$. Ignoring the seesaw contributions to eigenvalues, i.e. when $\left(* * / \lambda_{3}^{\prime}\right) \ll \lambda_{2}^{\prime}$ and $\left(\bullet \bullet / \lambda_{2}^{\prime}\right) \ll$ $\lambda_{1}^{\prime}$, we find that the three left-handed quark doublets $q_{j}$ $(j=1,2,3)$ are approximately

$$
q_{1}=\left(t_{L}, d_{L}\right), \quad q_{2}=\left(c_{L}, b_{L}\right), \quad q_{3}=\left(u_{L}, s_{L}\right),
$$

where $t_{L}, c_{L}$, and $u_{L}$ stand for left-handed components of the heaviest, middle, and lightest mass eigenstates of uptype quarks. The down-type mass eigenstates $b_{L}, s_{L}$, and $d_{L}$ are defined similarly. The CKM matrix for the up-type and down-type Yukawa matrices (17) is roughly

$$
V_{\mathrm{CKM}} \sim\left(\begin{array}{ccc} 
& 1 & \\
& & 1 \\
1 & &
\end{array}\right)
$$

corresponding to $\theta_{12} \sim \theta_{23} \sim \pi / 2$ and $\theta_{13} \sim 0$. This explains one of the $2^{3}$ combinations of peaks in Fig. 3. When the $\lambda_{3,2,1}^{\prime \prime / d}$ are found in different entries of the $3 \times 3$ Yukawa matrices, other peak combinations are obtained. Continuous distributions connecting $\theta_{i j} \sim 0$ to $\theta_{i j} \sim \pi / 2$ originate from the seesaw contributions that we have ignored. Thus the distributions become more and more localized around the peaks as $\lambda_{\max } / \lambda_{\min }$ is increased and seesaw contributions become less important. Therefore the small mixing angles of the observed CKM matrix are not atypical of distributions with large values of $\lambda_{\max } / \lambda_{\min }$.

How then do we interpret the peaks at $\pi / 2$ ? The flavor structure of the quark sector of the standard model is characterized by three general features. On the one hand, the quark masses have a hierarchical structure, and this is successfully reproduced. In addition, the $W$-boson current approximately connects three distinct pairs of quarks - we refer to this as "pairing structure," and it is also found in this landscape. Finally, the $W$-boson current connects pairs such that the lightest up-type quark is approximately paired with the lightest down-type quark, the middle up-type quark is approximately paired with the middle downtype, etc. We refer to this as the "generation structure" of the standard model. Mixing angles near $\pi / 2$ maximally violate this generation structure; for example, the set of angles $\theta_{12} \sim \theta_{23} \sim \pi / 2$ and $\theta_{13} \sim 0$ of (19) corresponds to the quark pairings in (18). There are 3 ! combinations in 

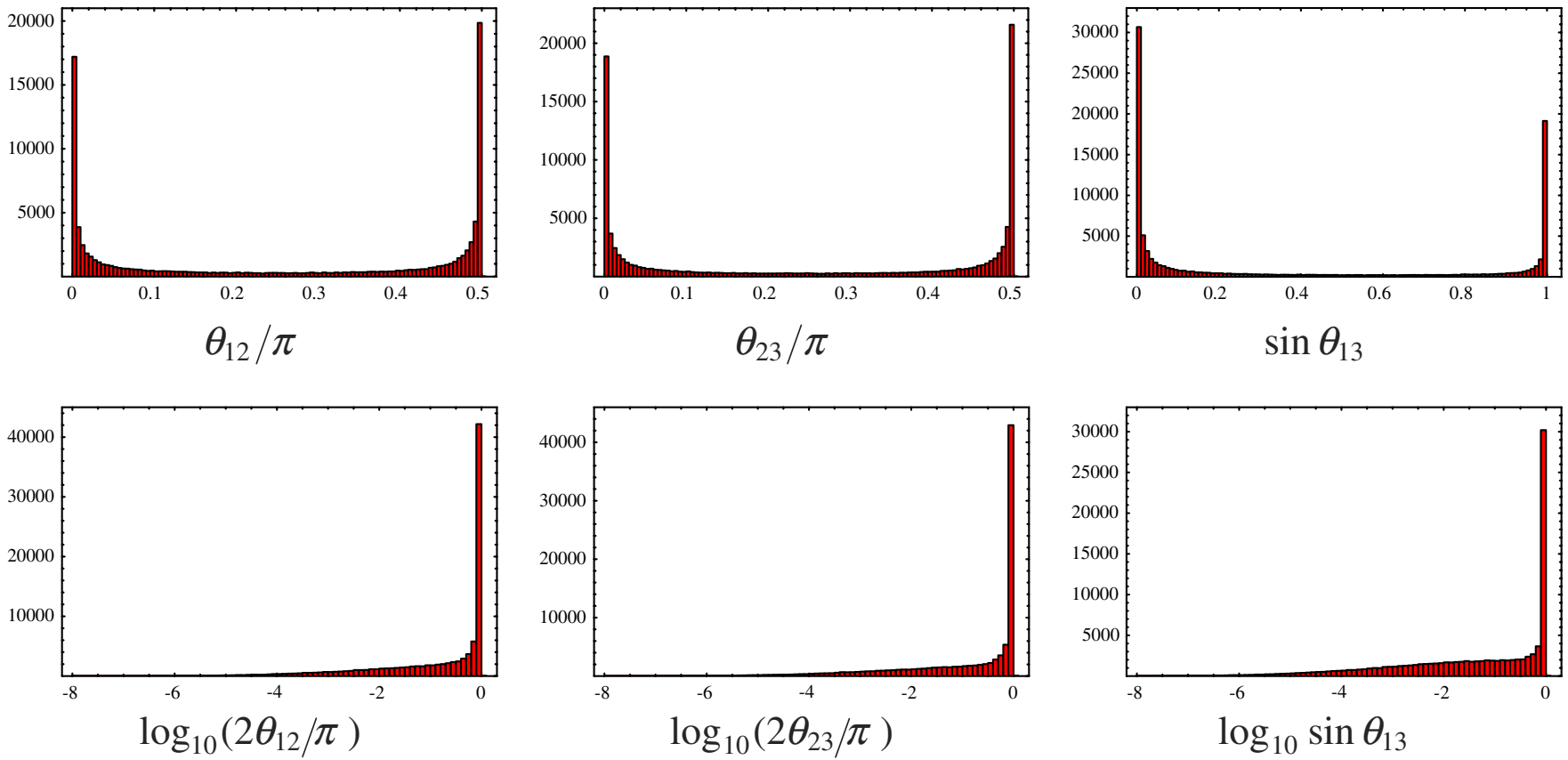

FIG. 3 (color online). Distributions of the three mixing angles of the CKM matrix that results from the distribution (4). The upper left panel is similar to Fig. 11 of [9]; however in [9] $\delta=1.16$ is used and the distribution is displayed only within the range $0 \leq \theta_{i j} \leq$ $\pi / 4$.

forming three pairs, only one of which has what we call the generation structure. 5

\section{Problems}

Is the existence of mixing-angle peaks about $\theta_{i j}=\pi / 2$ really a problem? The landscape that we have discussed so far may reproduce the generation structure of mass eigenstates in the $W$-boson current (when $\theta_{12} \sim \theta_{23} \sim \theta_{13} \sim 0$ ), but more often it does not. Although it might be argued that this is just a $1 / 3$ ! coincidence problem, it is still difficult to accept that the generation structure of flavor is not revealing something important about the underlying theory. It is also tempting to try to read something deeper from the observed hierarchy between $V_{u b} \sim \theta_{13} \sim 4 \times 10^{-3}$ and the Cabibbo angle $\theta_{12} \sim 0.2$. In this landscape this hierarchy is just a random statistical fluctuation (c.f. Figs. 2 and 3 ), and it does not appear this shortcoming can be overcome by a more ideal choice of $\lambda_{\max } / \lambda_{\min }$ (c.f. Fig. 1).

An even bigger problem is to understand how the probability distribution (4) arises or what is the correct distribution to replace it. That is, although the phenomenology of the landscape that we have considered may be deemed acceptable, we do not have a solid theoretical ground

\footnotetext{
${ }^{5}$ When $\sin \theta_{13} \sim 1, \theta_{12} \sim \theta_{23} \sim 0$ and $\theta_{12} \sim \theta_{23} \sim \pi / 2$ result in the same quark combinations. Likewise, $\left(\theta_{12}, \theta_{23}\right) \sim(0, \pi / 2)$ and $\left(\theta_{12}, \theta_{23}\right) \sim(\pi / 2,0)$ are the same. Therefore, there are only 3 ! physically different combinations, even though there are $2^{3}$ different ways to pick three peaks from Fig. 3.
}

upon which to base it. ${ }^{6}$ It is also obscure how the phenomenology of a lepton sector with large mixing angles and that of a quark sector with small mixing angles can be accommodated within a single theoretical framework. In the remaining sections of this paper we analyze some landscape models that successfully reproduce the phenomenology of hierarchy, pairing, and generation structure, while making progress on each of the four problems described above.

\section{A GAUSSIAN LANDSCAPE FOR QUARKS IN ONE EXTRA DIMENSION}

The landscape discussed in Sec. II assumes that all 18 elements of $\lambda^{u}$ and $\lambda^{d}$ are scanned independently. Yet without any correlation between these two Yukawa matri-

\footnotetext{
${ }^{6}$ Such an attempt is made in [13]. However, we consider that correlation among Yukawa couplings and the number of extra dimensions are crucial ingredients in understanding flavor physics, and these are missing in [13]. The intersecting D6-D6 system mentioned in [13] is dual to a $T^{3}$-fibered compactification of heterotic string theory and is simulated by the $D=3$ Gaussian landscape models of this article. Although [13] guesses that the scale-invariant (or nearly scale-invariant power-law) distribution might arise from the intersecting D-brane systems, we conclude otherwise. Specifically, we find that the scaleinvariant distribution (4) is derived from the $D=1$ Gaussian landscape model, but not a $D=3$ model. We also find that correlation among various elements of the Yukawa matrices is crucial to understanding flavor structure and the origin of generations.
} 
ces, generation structure will never be obtained. For example, in order to ensure that the heaviest up-type quark $t_{L}$ is contained in the same $\mathrm{SU}(2)_{L}$ doublet as the heaviest down-type quark $b_{L}$, we require the following. When an $(i$, $j$ ) element of the up-type Yukawa coupling $\lambda_{i j}^{u} \bar{u}_{i} q_{j} h$ is large, at least one of the three down-type Yukawa couplings $\lambda_{k j}^{d} \bar{d}_{k} q_{j} h^{*}(k=1,2,3)$ involving the same quark doublet $q_{j}$ should be large. A landscape of vacua must realize such a correlation between the up-type and downtype Yukawa matrices in order to explain the generation structure.

Perhaps one of the simplest ideas to introduce such a correlation is to introduce an extra dimension. If a large Yukawa coupling of $\lambda_{i j}^{u} \bar{u}_{i} q_{j} h$ is due to a substantial overlap of the wave functions of $q_{j}$ and $h$, then the down-type Yukawa couplings involving the same $q_{j}$ tend to be larger because of the overlap of $q_{j}$ and $h$. At the same time, for localized wave functions the overlap of some triplets of $\bar{u}$, $q$, and $h$ can be very small, and so there is hope to explain the hierarchically small Yukawa couplings necessary to account for light quarks.

In this section we present a simple toy landscape based on Gaussian wave functions spanning a circular extra dimension. Although a single extra dimension is introduced for simplicity, this model captures the essence of what one expects more generally from such "Gaussian landscapes" based on multiple extra-dimensional field theories. Through numerical simulation and an approximate analytical analysis, we find that the hierarchy, pairing, and generation structures of quarks is obtained statistically in this landscape. No flavor symmetry is needed.

\section{A. Emergence of scale-invariant distributions}

We introduce a single extra dimension with the simplest geometry: $S^{1}$. The wave functions for all of the quarks and the Higgs boson are assumed to be Gaussian with a common width $d$, and centered at arbitrary points on $S^{1}$ :

$$
\varphi\left(y ; y_{0}\right) \simeq \frac{1}{\pi^{1 / 4} \sqrt{M_{5} d}} e^{-\left(\left(y-y_{0}\right)^{2} / 2 d^{2}\right)} .
$$

Here $y$ is the coordinate of $S^{1}$ and $M_{5}$ is the cutoff scale of the effective field theory in $4+1$ dimensions. This wave function is normalized so that

$$
M_{5} \int_{0}^{L} d y \varphi^{2}(y)=1,
$$

where $L$ is the circumference of $S^{1}$. The wave function (20) should be made periodic on $S^{1}$, while maintaining the normalization in (21). Yet as long as the width of the Gaussian profile $d$ is parametrically smaller than the circumference $L$, the wave function is almost Gaussian. One should examine whether Gaussian wave functions arise as solutions to equations of motion of field theories in extra dimensions, but we defer this theoretical study to Sec. VII, and first study whether the assumption of Gaussian wave functions on extra dimensions leads to a successful explanation of the physics of quark and lepton masses and mixing angles.

We calculate the up-type and down-type Yukawa matrices with the overlap integrals

$$
\begin{aligned}
& \lambda_{i j}^{u}=g M_{5} \int_{S^{1}} d y \varphi_{i}^{\bar{u}}\left(y ; a_{i}\right) \varphi_{j}^{q}\left(y ; b_{j}\right) \varphi^{h}\left(y ; y^{h}\right), \\
& \lambda_{k j}^{d}=g M_{5} \int_{S^{1}} d y \varphi_{k}^{\bar{d}}\left(y ; c_{k}\right) \varphi_{j}^{q}\left(y ; b_{j}\right) \varphi^{h}\left(y ; y^{h}\right),
\end{aligned}
$$

where $g$ is an overall constant. ${ }^{7} \varphi_{j}^{q}(y), \varphi_{i}^{\bar{u}}(y), \varphi_{k}^{\bar{d}}(y)$, and $\varphi^{h}(y)$ are wave functions of left-handed quark doublets $q_{j}$ $(j=1,2,3)$, right-handed up-quarks $\bar{u}_{i}(i=1,2,3)$, righthanded down-quarks $\bar{d}_{k}(k=1,2,3)$ and of the Higgs boson, respectively, all of the Gaussian form (20). ${ }^{8}$ The center coordinates of these wave functions are $b_{j}, a_{i}, c_{k}$, and $y^{h}$, respectively. The matrices $\lambda^{u, d}$ are real, so that $C P$ is conserved in this toy landscape. Complex Gaussian wave functions will be studied in Sec. VI, where the effects of phases on the distributions of quark masses and mixing angles is found to be small. We assume that the center coordinates $b_{j}, a_{i}, c_{k}$, and $y^{h}$ are scanned freely and independently from one another on $S^{1}$. Because of the translational symmetry of $S^{1}$, only the relative difference between these center coordinates affects observables. Thus the effective number of scanning parameters is $n_{S}=9$. On the other hand, there are nine observables determined from the Yukawa matrices in the quark sector: six mass eigenvalues and three mixing angles. Thus the scanning parameters cover the space of observables and no precise prediction among the observables is available. However, since this Gaussian landscape covers the space of observables, our vacuum is unlikely to be missed in this ensemble.

The other model parameters, namely, the width $d$, circumference $L$, cutoff scale $M_{5}$, and coupling $g$, are treated

\footnotetext{
${ }^{7} \mathrm{We}$ will see in Sec. VII that these interactions may originate from super Yang-Mills interactions on a higher-dimensional spacetime, and then $g$ derives from the Yang-Mills coupling constant. Despite this origin, $g$ can be different in different sectors in effective descriptions such as those using just one extra dimension. For simplicity we set the constant $g$ to be the same for both the up and down sectors.

${ }^{8}$ In Sec. VII we study higher-dimensional supersymmetric field theories, where at short distances the up-type and downtype Yukawa couplings involve different Higgs doublets, $h_{1,2}$. Our results are independent of whether or not supersymmetry survives to the weak scale. If a supersymmetry survives compactification, we assume that the $h_{1,2}$ zero modes have the same localization. If no supersymmetry survives then we assume a single Higgs zero mode with a Gaussian profile. There is a " $\tan \beta$ " factor between the up and down sectors that we have set to unity. With weak-scale supersymmetry this factor arises from the ratio of Higgs vacuum expectation values, while with high scale supersymmetry breaking it arises from the composition of the light Higgs boson.
} 
as fixed. This treatment is quite arbitrary; among the myriad of other possibilities are to scan some or all of these parameters, to allow the up sector and the down sector to have different values of $g$, to choose different widths $d$ for different wave functions, etc. An extreme version of the landscape would allow everything to scan, leaving no fixed parameters to be input by hand. Our treatment — namely, four fixed parameters - is equivalent to slicing a specific subset out of a possibly much larger landscape. This allows us to identify a phenomenologically successful subset of the landscape and at the same time more easily explore its properties. It is a separate question whether this subset is typical of the full landscape, or if it is highly weighted because of cosmological evolution and/or environmental selection. Indeed, it is not implausible that the distributions of these four fixed parameters could be sharply peaked in the full landscape, since some toy landscapes predict Gaussian distributions for some parameters and cosmological evolution can provide exponentially steep weight factors. We consider that practical progress can be made by dividing the full problem into simpler parts.

Note that out of these four fixed parameters there are only two independent combinations that affect the Yukawa matrices. First, only the three dimensionless combinations, $g, M_{5} d$, and $M_{5} L$, are physical; the value of $M_{5}$ simply sets the scale for dimensionful parameters. Second, even as we change the "volume" of the extra dimension $M_{5} L$, the Yukawa couplings remain the same if the width-parameter $M_{5} d$ and the coupling $g$ are scaled so that the ratio $M_{5} d / M_{5} L=d / L$ and the effective coupling,

$$
g_{\text {eff }}=\frac{g}{\sqrt{M_{5} L}},
$$

remain the same. Therefore, the Yukawa couplings are effectively controlled by only these two parameters, $d / L$ and $g_{\text {eff }}$. (Note, however, that the volume $M_{5} L$ does affect the low-energy value of Newton's constant.)

The Yukawa couplings, given by the overlap integrals (22), can be expressed more explicitly in terms of the underlying parameters in a restricted region of the parameter space. Suppose that $d / L \ll 1$. Then the compactness of $S^{1}$ is not important in the calculation of the Yukawa couplings, as long as the center coordinates of quarks, $a_{i}$ (or $c_{k}$ ) and $b_{j}$, are close to that of the Higgs boson $y^{h}$ (which, by translational invariance, we set as the origin of the coordinate $y$ ). For such a vacuum, the Yukawa couplings are given by

$$
\begin{aligned}
\lambda_{i j}^{u} & \simeq g_{\mathrm{eff}}\left(\frac{4}{9 \pi} \frac{L^{2}}{d^{2}}\right)^{1 / 4} e^{-\left(1 / 3 d^{2}\right)\left(a_{i}^{2}+b_{j}^{2}-a_{i} b_{j}\right)}, \\
\lambda_{k j}^{d} & \simeq g_{\mathrm{eff}}\left(\frac{4}{9 \pi} \frac{L^{2}}{d^{2}}\right)^{1 / 4} e^{-\left(1 / 3 d^{2}\right)\left(c_{k}^{2}+b_{j}^{2}-c_{k} b_{j}\right)} .
\end{aligned}
$$

Let us compare this result to the form for the Yukawa couplings that results from approximate Abelian flavor symmetries (AFS). In the most general AFS scheme there is a small symmetry-breaking factor associated with each quark field, $\epsilon^{q, \bar{u}, \bar{d}}$, which leads to Yukawa matrix elements

$$
\lambda_{i j}^{u}=g_{i j} \epsilon_{i}^{\bar{u}} \epsilon_{j}^{q}, \quad \lambda_{k j}^{d}=g_{k j}^{\prime} \epsilon_{k}^{\bar{d}} \epsilon_{j}^{q},
$$

where the $g_{i j}$ and $g_{k j}^{\prime}$ are all of order unity. A mass hierarchy among the generations is realized by imposing $\epsilon_{3} \gg \epsilon_{2} \gg \epsilon_{1}$ in the left, right, or both sectors. Models with fewer parameters can be constructed and then the symmetry-breaking parameters are not all independent; consider, for example, a single Abelian symmetry with a symmetry-breaking parameter $\epsilon$ that appears in different entries with different powers due to generation dependent charges. Generation charges $(0,2,3)$ then give $\epsilon_{3}: \epsilon_{2}: \epsilon_{1}=$ $1: \epsilon^{2}: \epsilon^{3}$. No matter how the model is arranged, the mass hierarchy arises because the first generation feels much less flavor symmetry breaking than the third. Note that AFS theories are very flexible - any hierarchical pattern of fermion masses can be described by an appropriate AFS.

The result (24), which involves no flavor symmetry, has some similarities with the form (25). First notice that $a_{i}, b_{j}$, and $c_{k}$ can be both positive and negative and therefore the factor of $a_{i} b_{j}$ (and $c_{k} b_{j}$ ) in (24) is statistically neutral. Anticipating this statistical averaging, we cast (24) into the form of (25) with the identification

$$
\boldsymbol{\epsilon}_{i}^{\bar{u}}=e^{-\left(a_{i}^{2} / 3 d^{2}\right)}, \quad \boldsymbol{\epsilon}_{j}^{q}=e^{-\left(b_{i}^{2} / 3 d^{2}\right)}, \quad \boldsymbol{\epsilon}_{k}^{\bar{d}}=e^{-\left(c_{k}^{2} / 3 d^{2}\right)} .
$$

An important feature is that the AFS factor $\epsilon_{j}^{q}$ is shared by all elements of both the up-type and the down-type Yukawa couplings that involve the left-handed quark doublet $q_{j}$. This introduces a correlation between the up-type and down-type Yukawa matrices.

We first study the probability distribution for a single entry in the Yukawa matrix, ignoring correlations with other entries. This allows us to determine the analogue of (4) for this Gaussian landscape. Here we do not have a distinction between the up-type and down-type Yukawa matrices because we assume that the center coordinates of both the $\bar{u}$ and $\bar{d}$ wave functions are distributed randomly over $S^{1}$. The up-type Yukawa coupling in (24) is a function on the two-dimensional parameter space $(a, b) \in$ $[-L / 2, L / 2] \times[-L / 2, L / 2]$. The probability that the Yukawa coupling is larger than some value $\lambda_{0}$ is proportional to the area of an ellipse,

$$
\frac{(a+b)^{2}}{4}+\frac{3(a-b)^{2}}{4}=3 d^{2} \ln \left[\frac{g_{\text {eff }}}{\lambda_{0}}\left(\frac{4}{9 \pi} \frac{L^{2}}{d^{2}}\right)^{1 / 4}\right] .
$$

When the signs of $a$ and $b$ are opposite, the overlap of the three wave functions is small and the Yukawa coupling becomes small. This is why the region of $\lambda>\lambda_{0}$ is short in the $(a-b)$ axis and long in the $(a+b)$ axis. Comparing the area (27) to the total area $L^{2}$, we see the probability that $\lambda>\lambda_{0}$ is given by 


$$
P\left(\lambda>\lambda_{0}\right) \simeq 2 \sqrt{3} \pi\left(\frac{d}{L}\right)^{2} \ln \left[\frac{g_{\text {eff }}}{\lambda_{0}}\left(\frac{4}{9 \pi} \frac{L^{2}}{d^{2}}\right)^{1 / 4}\right],
$$

and hence the distribution is flat:

$$
\frac{d P(\lambda)}{d \ln \lambda}=2 \sqrt{3} \pi\left(\frac{d}{L}\right)^{2} \simeq 11\left(\frac{d}{L}\right)^{2}
$$

The distribution may cease to be flat as $a$ or $b$ approaches $\pm L / 2$, where the parameter space ends, because we ignored the periodic boundary condition in the calculation that led to (24). Setting this point aside we see that the probability distribution of Yukawa couplings in this toy landscape is flat on the $\ln \lambda$ axis and has an approximate span of

$$
\Delta \ln \lambda=\ln \left(\frac{\lambda_{\max }}{\lambda_{\min }}\right) \simeq \frac{1}{11}\left(\frac{L}{d}\right)^{2}
$$

arising from the inverse of the height of the distribution function (29). The overall hierarchy among Yukawa couplings $\Delta \ln \lambda=\ln \left(\lambda_{\max } / \lambda_{\min }\right)$ is proportional to $(L / d)^{2}$; the narrower the wave functions become, the further the wave functions can be separated, and the smaller the Yukawa couplings can be. As seen from (24), the upper end of this scale-invariant distribution $\lambda_{\max }$ is roughly $g_{\text {eff }} \sqrt{L / d}$. Note that the scale-invariant distribution (4) was introduced almost by hand in [9] in order to account for the large hierarchy among Yukawa couplings. It is interesting that this distribution is a natural prediction of our simple Gaussian landscape.

We performed a numerical calculation to confirm the semianalytical analysis above, taking account of the compactness of $S^{1}$ by making the wave function (20) periodic. The center coordinates of the quark wave functions $a_{i}, b_{j}$, $c_{k}$ were generated randomly $10^{5}$ times, and the Yukawa coupling was calculated through (22). This process was repeated for three different sets of the $\left(d / L, g_{\text {eff }}\right)$ parameters: $(0.08,1),(0.10,1)$, and $(0.14,1)$. The resulting distributions, shown in Fig. 4, are all roughly scale invariant (flat on a logarithmic scale), with heights proportional to $(d / L)^{2}$, just as we expected from the semianalytical discussion.

\section{B. Quark-sector phenomenology of the Gaussian landscape}

Let us now study the probability distributions of the mass eigenvalues and mixing angles. Figure 5 shows the result of a numerical simulation with $\left(d / L, g_{\text {eff }}\right)=$ $(0.08,0.2)$. The distributions of Yukawa eigenvalues in Fig. 5 are similar to those in Fig. 1, but with narrower distributions of $\lambda_{u, d}$ and with the distributions of $\lambda_{c, s}$ shifted downward. The prominent difference between the mixing-angle distributions in Fig. 5 and those in Fig. 3 is the absence of the unwanted peaks at $\theta_{i j} \simeq \pi / 2$. Thus we find the generation structure of the quark sector follows from this Gaussian landscape; introducing correlations between the up-type and down-type Yukawa matrix elements works perfectly. Moreover, in contrast to Fig. 3 the distribution of $\theta_{13}$ in Fig. 5 has a clear peak at $\theta_{13} \ll \mathcal{O}(1)$ when displayed on a logarithmic scale.

The distributions of Yukawa eigenvalues and mixing angles in Fig. 5 can be understood analytically if we allow ourselves to make an approximation. We have seen in Sec. III A that both the up-type and down-type Yukawa matrices have an AFS structure. Thus, we begin by determining the probability distribution of the AFS suppression factors $\epsilon^{q, \bar{u}, \bar{d}}$ in (26). The value of $\epsilon^{q}\left(\epsilon^{\bar{u}, \bar{d}}\right)$ is determined by the distance $|b|(|a|,|c|)$ of the left-handed (righthanded) quark wave function from the Higgs boson wave function. Since the center coordinates are scanned randomly over the extra dimension $S^{1}$, the probability measure is

$$
d P(b)=\frac{2}{L} d|b|, \quad \text { for } 0 \leq|b| \leq \frac{L}{2} .
$$

The measure for the right-handed quarks is the same, and we only deal with the left-handed quarks hereafter. Converting the variable from $|b|$ to $\ln \epsilon^{q}$ using (26), we find

$$
d P(y)=\frac{d y}{2 \sqrt{y}}, \quad \text { for } 0 \leq y \leq 1,
$$

where $y \equiv \ln \epsilon^{q} / \Delta \ln \epsilon$ and we have defined $\Delta \ln \epsilon \equiv$ $-(L / 2 d)^{2} / 3=-(L / d)^{2} / 12$.

The center coordinates of the three left-handed quark wave functions are chosen randomly, thus three AFS sup-
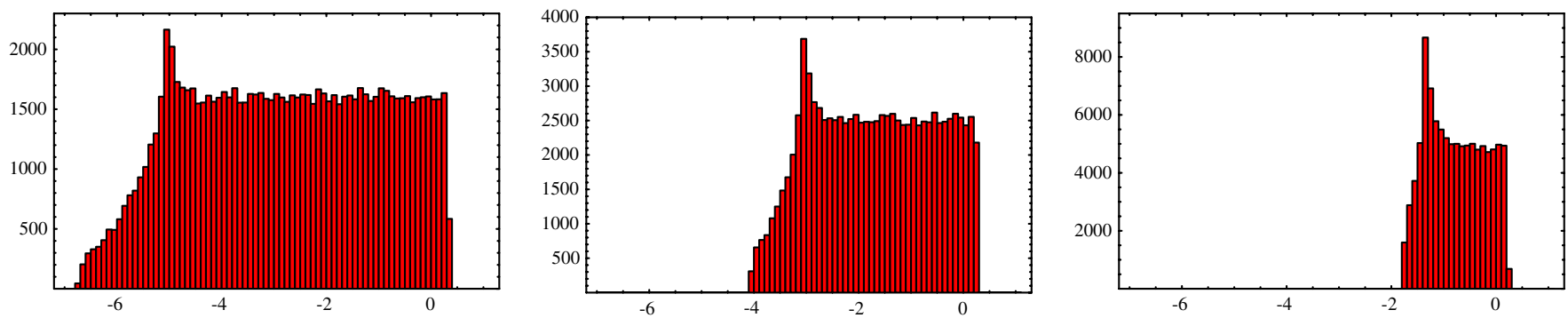

FIG. 4 (color online). Distributions of $10^{5}$ Yukawa matrix elements, generated from the Gaussian landscape on $S^{1}$. From left to right the panels correspond to $\left(d / L, g_{\text {eff }}\right)=(0.08,1),(0.1,1)$, and $(0.14,1)$. 

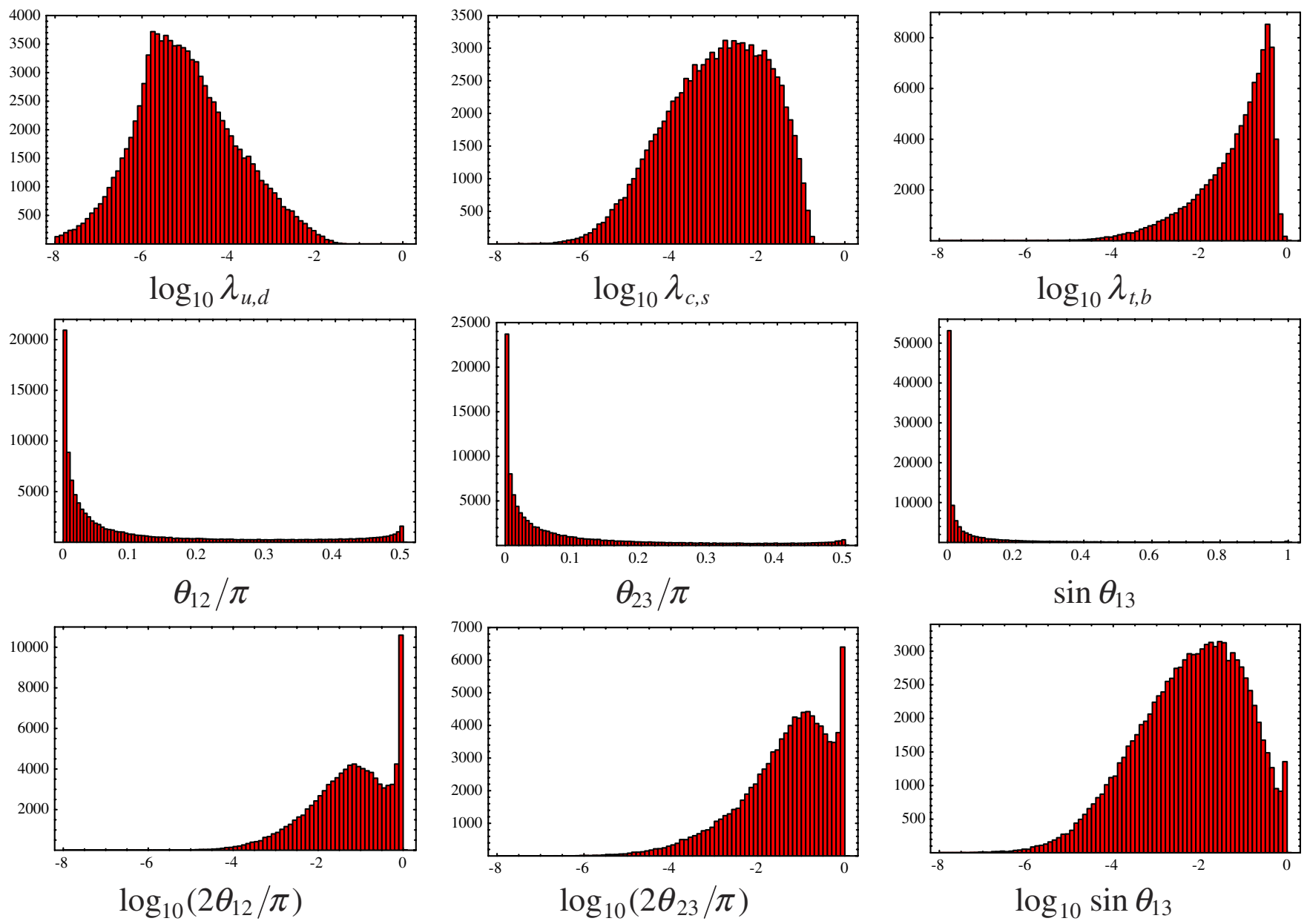

FIG. 5 (color online). Distributions of three quark Yukawa eigenvalues and mixing angles, based on a numerical simulation of the Gaussian landscape on $S^{1}$, using $\left(d / L, g_{\text {eff }}\right)=(0.08,0.2)$.

pression factors follow (32) independently. The smallest of these corresponds to the suppression factor $\epsilon_{1}$ for the lightest quark, while the middle factor $\epsilon_{2}$ and largest factor $\epsilon_{3}$ correspond to the middle and the heaviest quarks. The distribution of $\epsilon_{1,2,3}$ is given by

$$
\begin{gathered}
d P\left(y_{1}, y_{2}, y_{3}\right)=\frac{3 !}{2^{3}} \frac{d y_{1} d y_{2} d y_{3}}{\sqrt{y_{1} y_{2} y_{3}}} \Theta\left(y_{1}-y_{2}\right) \Theta\left(y_{2}-y_{3}\right), \\
\text { for } 0 \leq y_{i} \leq 1,
\end{gathered}
$$

where $y_{i} \equiv \ln \epsilon_{i} / \Delta \ln \epsilon$. Note that $y_{1}>y_{2}>y_{3}$. The distributions of the individual AFS suppression factors $\epsilon_{1,2,3}$ are obtained by integrating (33) with respect to the other two variables:

$$
\begin{gathered}
d P\left(y_{3}\right)=\frac{3}{2} \frac{\left(1-\sqrt{y_{3}}\right)^{2}}{\sqrt{y_{3}}} d y_{3}, \\
d P\left(y_{2}\right)=3\left(1-\sqrt{y_{2}}\right) d y_{2}, \quad d P\left(y_{1}\right)=\frac{3}{2} \sqrt{y_{1}} d y_{1} .
\end{gathered}
$$

These distribution functions are shown in Fig. 6. Meanwhile, their averages are given by

$$
\begin{aligned}
\frac{\left\langle\ln \epsilon_{3}\right\rangle}{\Delta \ln \epsilon}=\left\langle y_{3}\right\rangle=0.1, & \frac{\left\langle\ln \epsilon_{2}\right\rangle}{\Delta \ln \epsilon}=\left\langle y_{2}\right\rangle=0.3, \\
\frac{\left\langle\ln \epsilon_{1}\right\rangle}{\Delta \ln \epsilon}=\left\langle y_{1}\right\rangle=0.6, & \frac{\left\langle\ln \left(\epsilon_{1} / \epsilon_{2}\right)\right\rangle}{\left\langle\ln \left(\epsilon_{2} / \epsilon_{3}\right)\right\rangle}=1.5 .
\end{aligned}
$$

In a sense, this Gaussian landscape predicts the ratio of the AFS charges for the three generations: 6:3:1. However this ratio only describes the statistical ensemble, and the distribution functions (34) contain more information.

The distributions of Yukawa eigenvalues follow from (34) with the approximation

$$
\ln \left(\lambda_{i} / \lambda_{\max }\right) \sim \ln \epsilon_{i}^{q} \epsilon_{i}^{\bar{q}}
$$

Explicit expressions of the distribution functions derived in this way are found in the appendix, (A1)-(A3), and are plotted in Fig. 6. From (A1)-(A3) we see that for small values of $z_{i} \equiv \ln \left(\lambda_{i} / \lambda_{\max }\right) / \Delta \ln \epsilon$ (these correspond to large eigenvalues), the distribution functions behave as $\neq 0, \propto z_{2}$, and $\propto z_{1}^{2}$, which is confirmed in the numerical simulation in Fig. 5. Note that the distribution of $\lambda_{s, c}$ begins at around $10^{-1}$, and that of $\lambda_{u, d}$ at around $10^{-2}$, 
contrary to the approximate analytic distribution functions (A2) and (A3), which begin at $\mathcal{O}(1)$. This is probably due to the effects of diagonalizing the Yukawa matrices. As is familiar in quantum mechanics, two degenerate eigenvalues split even in the presence of the slightest perturbation. We later refer to this effect as the "diagonalization effect."

We should also note that the AFS approximation (24) and (26) breaks down for small values of Yukawa eigenvalues. The distributions based on the AFS approximation extend all the way down to $\ln \left(\lambda / \lambda_{\max }\right) \sim 2 \Delta \ln \epsilon$, while the numerical results cover a logarithmic range closer to (30). Since $\Delta \ln \epsilon \simeq-\Delta \ln \lambda$, the analytically derived range of $\ln \lambda$ covers twice the logarithmic scale that we expect. This discrepancy arises from the compactness of the extra dimension. That is, what really matters in the exponent of (24) is

$$
\begin{aligned}
& \min \left[(a+n L)^{2}+(b+m L)^{2}-(a+n L)(b+m L)\right], \\
& \quad \text { for } n, m \in \mathbb{Z} .
\end{aligned}
$$

As the center coordinates $a$ and $b$ approach $\pm L / 2$, nonzero choices of $n$ and $m$ may become just as important as $n=$ $m=0$ in (24). Indeed, when $|a| \sim|b| \sim L / 2$ integers $n$ and $m$ can be chosen so that the last term is negative. When the compactness of the extra dimension is taken into account, the full expression (37) cannot be larger than $(L / 2)^{2}$. This is why the distributions of Yukawa couplings and eigenvalues in Figs. 4 and 5 span over $\ln \left(\lambda / \lambda_{\max }\right) \sim$ $\Delta \ln \epsilon$ in the numerical results. The AFS approximation breaks down because the coefficient $g_{i j}=$ $e^{\left(a_{i}+n L\right)\left(b_{j}+m L\right) / 3 d^{2}}$ is not statistically neutral when $\left|a_{i}\right|$ and $\left|b_{j}\right|$ are around $L / 2$.

Distribution functions of the mixing angles can also be obtained by pursuing the AFS approximation, along with the additional (crude) approximations:

$$
\begin{aligned}
& \ln V_{u s} \sim \ln \left(\max \left\{\left(\epsilon_{1}^{q} / \epsilon_{2}^{q}\right)_{\mathrm{u}-\text { sector }},\left(\epsilon_{1}^{q} / \epsilon_{2}^{q}\right)_{\mathrm{d}-\text { sector }}\right\}\right), \\
& \ln V_{c b} \sim \ln \left(\max \left\{\left(\epsilon_{2}^{q} / \epsilon_{3}^{q}\right)_{\mathrm{u}-\text { sector }},\left(\epsilon_{2}^{q} / \epsilon_{3}^{q}\right)_{\mathrm{d} \text {-sector }}\right\}\right),
\end{aligned}
$$

$$
\ln V_{u b} \sim \ln \left(\max \left\{\left(\epsilon_{1}^{q} / \epsilon_{3}^{q}\right)_{\mathrm{u}-\text { sector }},\left(\epsilon_{1}^{q} / \epsilon_{3}^{q}\right)_{\mathrm{d}-\text { sector }}\right\}\right) .
$$

Explicit expressions are found in (A5)-(A7) and are plotted in Fig. 6. These approximate analytic distribution functions capture qualitative features of the numerical results. Note that the distribution function of $\sin \theta_{13}$ becomes zero at $\log _{10} \sin \theta_{13} \sim 0$ because $\sin \theta_{13}$ can be of order unity only when all three eigenvalues are almost degenerate in either the up- or down sector, and the probability for this to occur is small. The averages of the mixing angles in a logarithm scale are ordered

$$
\left\langle\theta_{13}\right\rangle<\left\langle\theta_{12}\right\rangle<\left\langle\theta_{23}\right\rangle,
$$

both in the numerical simulation and in the analytic distributions; see (A8). This is regarded as a consequence of the AFS charges in (35); indeed in (35) we have

$$
\left\langle\ln \left(\epsilon_{1} / \epsilon_{3}\right)\right\rangle<\left\langle\ln \left(\epsilon_{1} / \epsilon_{2}\right)\right\rangle<\left\langle\ln \left(\epsilon_{2} / \epsilon_{3}\right)\right\rangle .
$$

Whether the assignments in (35) are observationally acceptable or not is debatable, and we will return to this issue in Sec. IV. We discuss in Sec. V how the "charge assignment" changes when the geometry $S^{1}$ is replaced by other geometries.

To summarize, we see that the qualitative expectations from an AFS-type mass matrix hold true in this landscape. In particular, the similarities between the landscape generated Yukawa couplings (24) and those of (25) allow us to understand Gaussian landscapes, at least to some degree, using intuition based on models of AFS. Of course, between these approaches the origin of small parameters is completely different: in the landscape they arise from small overlaps of wave functions well separated in the extra dimension, whereas in AFS they arise from small symmetry-breaking parameters. Let us now emphasize this distinction.

A crucial general feature of all AFS models is that the mass hierarchy between generations, $m_{3} \gg m_{2} \gg m_{1}$, arises because there is a hierarchy in the amount of symmetry breaking coupled to these generations. This is true in
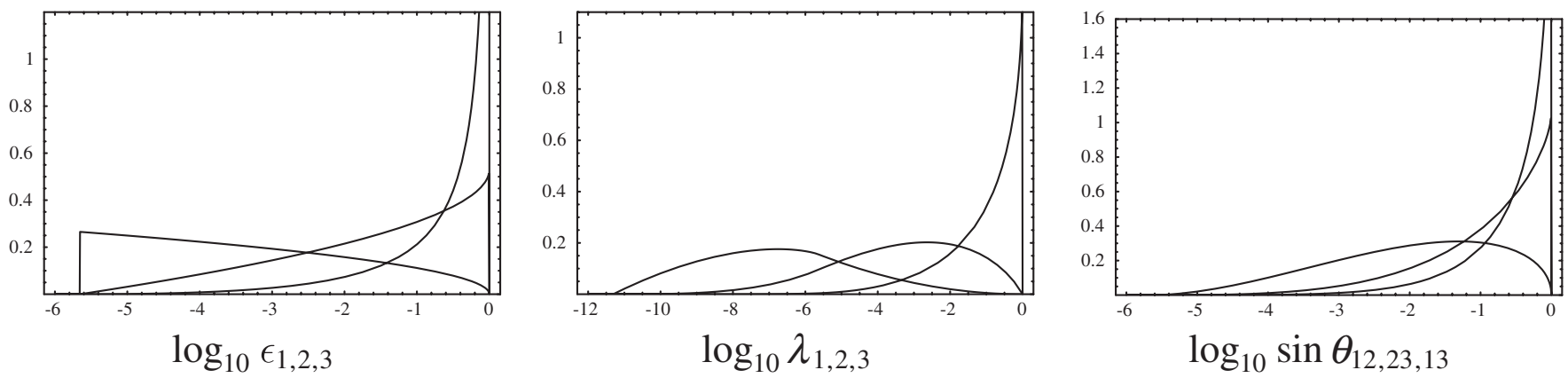

FIG. 6. Distributions of the three AFS suppression factors, the three Yukawa eigenvalues, and the CKM mixing angles; the latter calculated naively from (36) and (38)-(40) using the Gaussian landscape on $S^{1}$. The CKM mixing angle $\theta_{23}$ is the one most sharply peaked at zero, while $\theta_{13}$ is most spread out. These figures correspond to $d / L=0.08$ and thus $\Delta \log _{10} \epsilon=5.65$. Recall that these distribution functions are not reliable for small eigenvalues. 
the general Abelian case by the choice $\epsilon_{3} \gg \epsilon_{2} \gg \epsilon_{1}$. In more restricted versions having $\epsilon_{i} \approx \epsilon^{Q_{i}}$, the hierarchy is imposed by a choice of charges $Q_{1}>Q_{2}>Q_{3}$. If the flavor symmetry is non-Abelian, then there is a hierarchy of symmetry breaking, for example, the rank may be broken from $i$ to $i-1$ with strength $\epsilon_{i}$. Thus AFS can in principle describe any flavor pattern, for example, one heavy generation with Yukawas of order unity and two very light generations with Yukawas of order $10^{-10}$. The situation with this Gaussian landscape is very different. Each Yukawa coupling is a statistical quantity, with a probability distribution that is approximately scale invariant over a range determined by a single small parameter, $d / L$. Relative to this range, the hierarchy of mass eigenvalues, including the typical intergenerational mass ratios and mixing angles, arises purely from statistics. Unlike with the AFS parameters $\epsilon_{i}$, there is no sense in which the fundamental theory distinguishes among generations. Therefore unlike with AFS, this Gaussian landscape cannot accommodate one heavy generation and two very light generations of comparable mass. Within statistical uncertainties, the landscape determines the AFS charges.

\section{Environmental selection effects}

It is a formidable task to understand all of the environmental effects that would be associated with a landscape scanning over the flavor parameters of the standard model. Furthermore, without a specific theory for the landscape it is unclear whether certain qualitative features of the flavor sector arise due to environmental selection, due to systematic features of the landscape distributions, or due to accident. Consider these examples. In the Gaussian landscape of this section, $\lambda_{1} / \lambda_{2}$ tends to be smaller than $\lambda_{2} / \lambda_{3}$. Therefore if $d / L$ is chosen so as to explain the hierarchy $\lambda_{2} / \lambda_{3}$, then the relative lightness of the up and down quarks is explained. The value of $d / L$ required to reproduce the quark masses we measure may be selected dynamically within the fundamental theory or it may be selected due to environmental effects associated with having light up and down quarks. Likewise, the unexpected hierarchy $m_{t} / m_{b}$ may be due to the dynamical or accidental selection of different effective coupling constant $g_{\text {eff }}$ 's for the up and down sectors, ${ }^{9}$ or environmental selection could favor a very large top mass as described in [7].

We now describe qualitative effects associated with one possibility for environmental selection, which is the selection of a large top mass to ensure the stability of our present Higgs phase [7]. Consider a simple cut on the top Yukawa:

$$
\log _{10} \lambda_{t} \geq-0.3
$$

\footnotetext{
${ }^{9}$ This hierarchy may also be due to weak-scale supersymmetry with a large $\tan \beta$; however this requires that the wave functions for the up-type and down-type Higgs bosons are located at the same position in the extra dimension.
}

We emphasize that we impose this $t$-cut to study qualitative effects; it is not intended to be precise. We first study the distributions of the quark Yukawa eigenvalues, which follow the distributions shown in Fig. 7. The three distributions in the first row correspond to Yukawa eigenvalues before the environmental cut is imposed. Since our landscape has not introduced any difference between the upand down-type sectors, the three distributions are the same in both sectors. Imposing the $t$-cut (43) reduces the sample to $5 \%$ of its original size. After the $t$-cut is imposed, the eigenvalue distributions are modified into those displayed in the second and third rows of Fig. 7. A notable effect of the $t$-cut is that the distributions of the other Yukawa eigenvalues are dragged upward. This effect is more evident in the up sector than in the down sector, improving the fit of $\lambda_{b} / \lambda_{t}, \lambda_{c} / \lambda_{b}$, and $\lambda_{s} / \lambda_{c}$.

One might consider that this improvement is not enough. Certainly the $\lambda_{b} / \lambda_{t}$ prediction is improved, but we find that the standard model value only moves from the 3rd to about the 6th percentile. Furthermore, the typical value of $\lambda_{b}$ is large even without the $t$-cut, and the distribution of $\lambda_{b}$ is shifted upward after this cut is imposed. There are a number of ways to modify the Gaussian landscape of this section to alleviate this problem. Some of these have already been mentioned; others are presented in Sec. V, where we study how the geometry of the extra dimensions affects the distributions of observables in Gaussian landscapes.

The effects of the $t$-cut are also seen in the distributions of the mixing angles; see Fig. 8. The probability that $\theta_{23} \sim$ $\mathcal{O}(1)$ is reduced significantly, which is understandable because $\lambda_{t}$ (and hence $\lambda_{b}$ ) tends to be very close to $\mathcal{O}(1)$ in the sample passing the $t$-cut, while $\lambda_{c}$ and $\lambda_{s}$ are rarely larger than $10^{-1}$. The $\theta_{23}$ distribution is reduced at small values as well, probably because the distributions of $\lambda_{c}$ and $\lambda_{s}$ are reduced at small values. Thus environmental selection on some flavor parameters modifies the distributions of other flavor parameters through a complicated chain of correlations.

\section{Summary}

We introduce a simple Gaussian landscape, based on a more microscopic description of the origin of flavor involving a single extra dimension, which predicts probability distributions for the $C P$-conserving quark-sector flavor parameters. Using only two free parameters, $g_{\text {eff }}$ and $d / L$, this restrictive theory provides as good a fit to the flavor parameters as conventional theories of flavor based on flavor symmetries. This is especially so when the Gaussian landscape is augmented by environmental selection in favor of large top-Yukawa coupling. This Gaussian landscape is characterized by the homogeneous scanning of the center coordinates of Gaussian wave functions in the extra dimension, which results in homogeneous scanning of Yukawa matrix elements on a logarithmic scale. The 

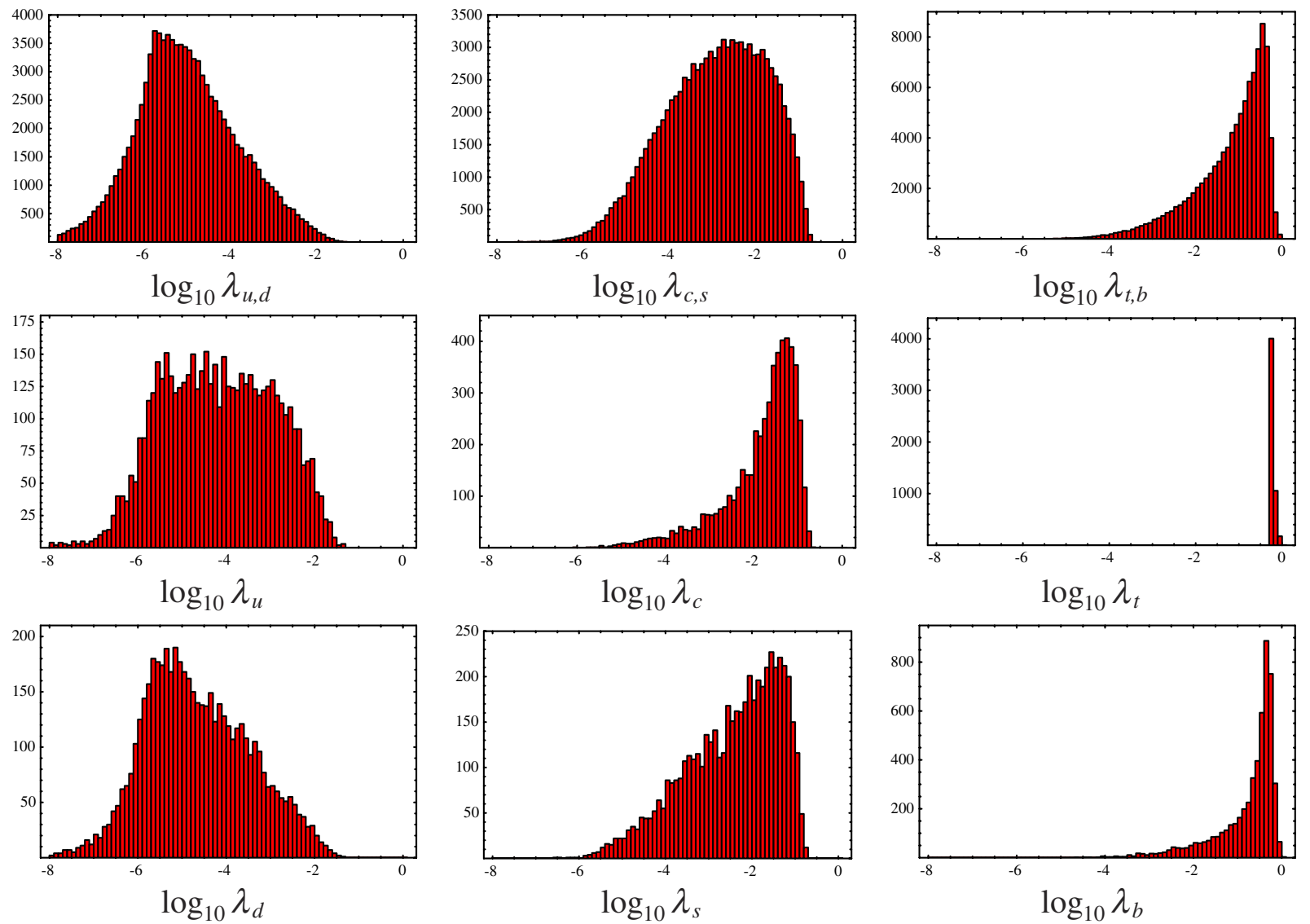

FIG. 7 (color online). Distributions of quark Yukawa eigenvalues based on the Gaussian landscape on $S^{1}$. The first row shows the distributions from Fig. 5 before the $t$-cut is imposed. The last two rows show the distributions of the roughly $5 \%$ of Yukawa matrices that pass the $t$-cut.
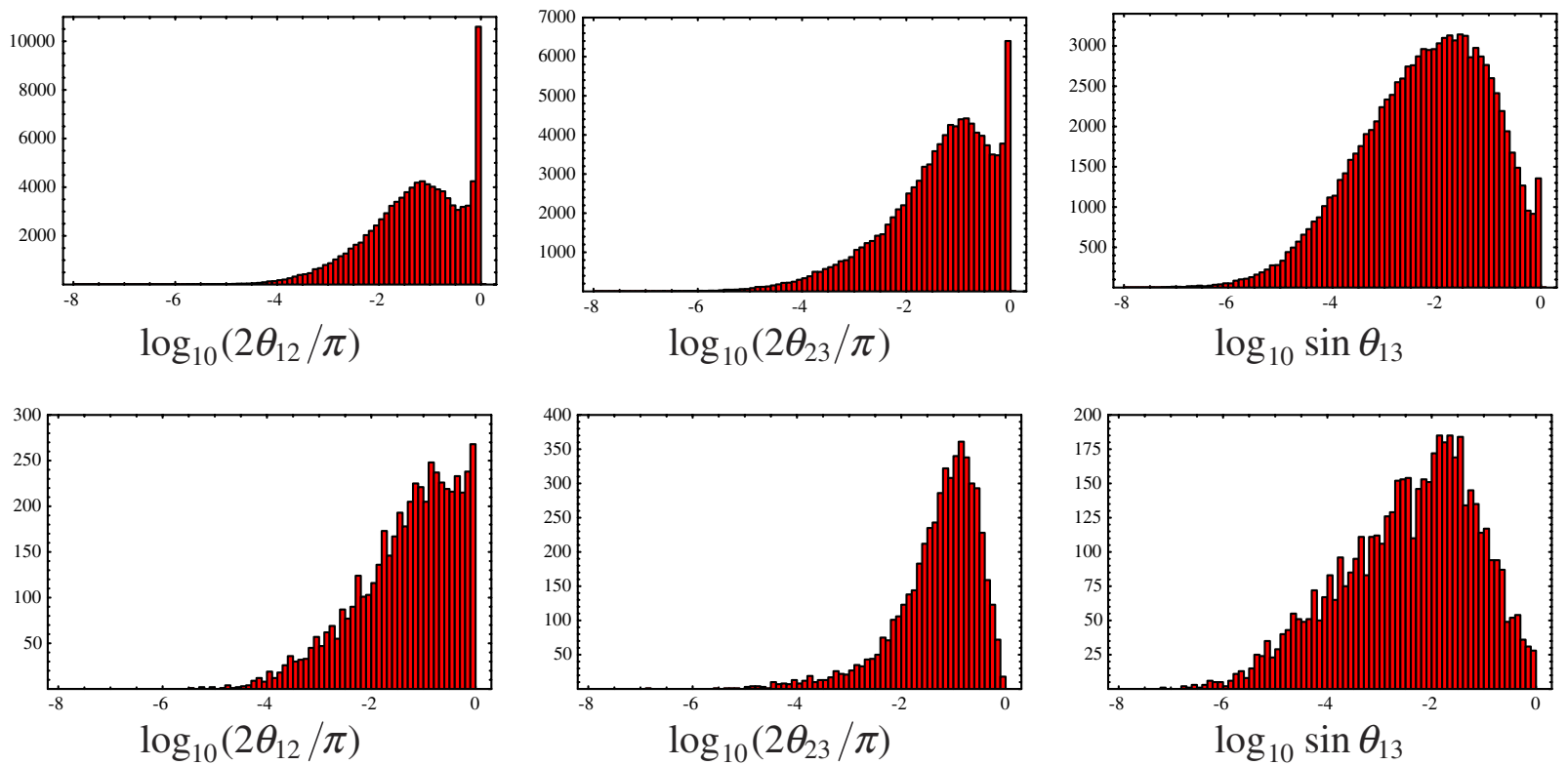

FIG. 8 (color online). Distributions of CKM mixing angles, based on the Gaussian landscape on $S^{1}$. The first row shows the distributions before the $t$-cut is imposed, while the second row shows the distributions corresponding to the roughly $5 \%$ of Yukawa matrices that pass the $t$-cut. 
three eigenvalues of each Yukawa matrix then tend to be well separated from one another on the logarithmic scale. Thus, the hierarchical structure of quark mass eigenvalues is obtained. The wave functions of the three quark doublets $q_{j}$ and the Higgs boson $h$ are assumed to be quite localized in the extra dimension, and the overlap of these wave functions introduces correlation between the up-type and down-type Yukawa matrices. This is the essence behind (the microscopic explanation) for the generation structure of the quark sector, that is the phenomenon that the heaviest (middle and lightest) up-type quark is coupled to the heaviest (middle and lightest) down-type quark in the $W$-boson current.

\section{GOODNESS-OF-FIT TESTS OF LANDSCAPE MODELS}

Before proceeding to generalize the Gaussian landscape, we here take an aside to discuss some possibilities for testing theories that assume a huge landscape of vacua. A landscape theory that contains the observed vacuum is not directly falsifiable in the convention sense, since we can only compare one value of any "variable" (the value in our universe) to its predicted distribution. On the other hand, with a statistical theoretical prediction we can at least calculate the probability to have measured a more atypical value for a landscape variable. If this probability is small, the landscape can be excluded at a certain confidence level. Indeed, the more numerous are the predictions of a landscape theory, the greater the possibility to exclude the theory. Consider, for example, quantum theory, where statistical theoretical predictions are well tested using scattering experiments that count the event rates in a large number of bins/modes. The parameterization of the standard model is dominated by the 22 flavor parameters, 20 of which have either been measured or may be measured in the near future. Therefore it seems that the flavor structure of the standard model provides the best realm in which to test landscape theories.

In this section we study two statistical tests, the chisquare statistic and the p-value statistic, to illustrate how Gaussian landscapes may be excluded by experiment. As this section is intended to be only illustrative, we consider only the quark sector as described by the Gaussian landscape on $S^{1}$ (which in this section is referred to as the $S^{1}$ model), and we do not consider any weight factors that may arise due to cosmological evolution or environmental selection effects. The existence of correlations among the distributions of flavor parameters in Gaussian landscapes allows for a rich statistical analysis, and the discussion below only scratches the surface.

\section{A. The chi-square statistic}

Our first example is to use the so-called chi-square statistic as a measure of goodness-of-fit between the observed flavor parameters and their hypothetical landscape distributions. The $C P$-conserving flavor parameters in the quark sector consist of the nine variables:

$$
\begin{aligned}
& \left\{X_{I}\right\} \equiv \log _{10}\left\{\lambda_{u}, \lambda_{c}, \lambda_{t}, \lambda_{d}, \lambda_{s}, \lambda_{b}, \theta_{12}, \theta_{23}, \sin \theta_{13}\right\}, \\
& \quad \text { for } I=1, \cdots, 9,
\end{aligned}
$$

with the logarithm acting on every element of the list. The CKM phase is not included in this list because the $S^{1}$ model we consider here is $C P$-conserving. The chi-square statistic is

$$
\chi^{2}=\sum_{I, J}\left(\hat{X}_{I}-\left\langle X_{I}\right\rangle\right)\left(V^{-1}\right)_{I J}\left(\hat{X}_{J}-\left\langle X_{J}\right\rangle\right),
$$

where hats denote the measured value of a parameter. The values listed in Table I are used for $\left\{\hat{X}_{I}\right\}$. Brackets denote the landscape average, and the covariance matrix $V$ is given by

$$
V_{I J}=\operatorname{Cov}\left(X_{I}, X_{J}\right)=\left\langle X_{I} X_{J}\right\rangle-\left\langle X_{I}\right\rangle\left\langle X_{J}\right\rangle .
$$

The chi-square statistic is invariant under any linear transformation among the variables $\left\{X_{I}\right\}$.

The correlation among the parameters in (44) cannot be ignored. Specifically, for the three mass eigenvalues in the up sector, the correlation matrix, $\rho_{I J} \equiv V_{I J} / \sqrt{V_{I I} V_{J J}}$, is given by

$$
\rho_{I J}=\left(\begin{array}{ccc}
1 . & 0.57 & 0.39 \\
0.57 & 1 . & 0.58 \\
0.39 & 0.58 & 1 .
\end{array}\right), \quad \text { for } I, J=1,2,3 .
$$

Note that the off-diagonal terms are not negligible compared to the diagonal terms. The positive correlations are as expected from the combined probability distribution (33) of the AFS suppression factors $\log _{10} \epsilon_{i}^{q, \bar{u}}$. Meanwhile, the correlation between the up and down-sector mass eigenvalues, $\left\{\log _{10} \lambda_{u}, \log _{10} \lambda_{c}, \log _{10} \lambda_{t}\right\} \times\left\{\log _{10} \lambda_{d}, \log _{10} \lambda_{s}\right.$, $\left.\log _{10} \lambda_{b}\right\}$, is given by

$$
\begin{aligned}
\rho_{I J}= & \left(\begin{array}{lll}
0.32 & 0.27 & 0.20 \\
0.27 & 0.39 & 0.29 \\
0.20 & 0.29 & 0.43
\end{array}\right) \\
& \text { for } I=1,2,3 \text { and } J=4,5,6 .
\end{aligned}
$$

This is also sizable and positive, confirming that the mass eigenvalues in the down sector are dragged upward under the $t$-cut. The mixing angles are also correlated with mass hierarchy; the correlation matrix between $\log _{10}\left\{\lambda_{u} / \lambda_{c}, \lambda_{c} / \lambda_{t}, \lambda_{u} / \lambda_{t}\right\}$ and $\log _{10}\left\{\theta_{12}, \theta_{23}, \sin \theta_{13}\right\}$ is given by

$$
\rho_{I J}=\left(\begin{array}{ccc}
0.34 & -0.14 & 0.28 \\
-0.21 & 0.42 & 0.14 \\
0.16 & 0.21 & 0.38
\end{array}\right),
$$

where now $I=X_{1}-X_{2}, X_{2}-X_{3}, X_{1}-X_{3}$ and $J=7,8$, 9. We see that larger mass hierarchies are correlated with smaller mixing angles. 
TABLE I. The 17 measured flavor parameters and a limit on a mixing angle in the lepton sector. All data comes from the pdgLive feature from the Particle Data Group [14]. For comparison to distributions provided throughout this paper, the quark and charged-lepton Yukawa eigenvalues and the CKM matrix elements have been run up to the (reduced) Planck scale $M_{P}=2.4 \times 10^{18} \mathrm{GeV}$, assuming no physics beyond the standard model enters up to this scale. On the other hand, the renormalization group scaling effects are not taken into account in the neutrino sector.

\begin{tabular}{lccc}
\hline \hline$\lambda_{u}$ & $(3.0 \pm 1.0) \times 10^{-6}$ & $\log _{10} \lambda_{u}$ & $-5.53_{-0.17}^{+0.13}$ \\
$\lambda_{c}$ & $(1.4 \pm 0.1) \times 10^{-3}$ & $\log _{10} \lambda_{c}$ & $-2.87 \pm 0.03$ \\
$\lambda_{t}$ & $(4.9 \pm 0.3) \times 10^{-1}$ & $\log _{10} \lambda_{t}$ & $-0.31 \pm 0.02$ \\
$\lambda_{d}$ & $(6.7 \pm 2.7) \times 10^{-6}$ & $\log _{10} \lambda_{d}$ & $-5.18_{-0.22}^{+0.15}$ \\
$\lambda_{s}$ & $(1.3 \pm 0.4) \times 10^{-4}$ & $\log _{10} \lambda_{s}$ & $-3.90_{-0.13}^{+0.10}$ \\
$\lambda_{b}$ & $(5.7 \pm 0.1) \times 10^{-3}$ & $\log _{10} \lambda_{b}$ & $-2.24 \pm 0.01$ \\
$\frac{1}{\pi} \theta_{12}^{\mathrm{CKM}}$ & $(7.31 \pm 0.03) \times 10^{-2}$ & $\log _{10}\left(\frac{2}{\pi} \theta_{12}^{\mathrm{CKM}}\right)$ & $-0.835 \pm 0.002$ \\
$\frac{1}{\pi} \theta_{23}^{\mathrm{CKM}}$ & $\left(1.344_{-0.025}^{+0.003}\right) \times 10^{-2}$ & $\log _{10}\left(\frac{2}{\pi} \theta_{23}^{\mathrm{CKM}}\right)$ & $-1.571_{-0.008}^{+0.001}$ \\
$\sin \theta_{13}^{\mathrm{CKM}}$ & $(4.01 \pm 0.09) \times 10^{-3}$ & $\log _{10}\left(\sin \theta_{13}^{\mathrm{CKM}}\right)$ & $-2.397 \pm 0.010$ \\
$\frac{1}{\pi} \delta^{\mathrm{CKM}}$ & $0.29-0.34$ & & -5.52 \\
$\lambda_{e}$ & $2.99 \times 10^{-6}$ & $\log _{10} \lambda_{e}$ & -3.20 \\
$\lambda_{\mu}$ & $6.31 \times 10^{-4}$ & $\log _{10} \lambda_{\mu}$ & -1.97 \\
$\lambda_{\tau}$ & $1.07 \times 10^{-2}$ & $\log _{10} \lambda_{\tau}$ & \\
$\frac{1}{\pi} \theta_{12}^{\mathrm{PMNS}}$ & $0.19 \pm 0.01$ & $\sin \left(2 \theta_{12}^{\mathrm{PMNS}}\right)$ & $0.93_{-0.02}^{+0.01}(90 \% \mathrm{C} . \mathrm{L})$. \\
$\frac{1}{\pi} \theta_{23}^{\mathrm{PMNS}}$ & $0.20-0.30$ & $\sin \left(2 \theta_{23}^{\mathrm{PMNS}}\right)$ & $0.96-1.0(90 \% \mathrm{C} . \mathrm{L})$. \\
$\sin \theta_{13}^{\mathrm{PMNS}}$ & $<0.18(95 \% \mathrm{CL})$ & $\log _{10}\left(\sin _{13}^{\mathrm{PMNS}}\right)$ & $<-0.74$ \\
$\Delta m_{\mathrm{atm}}^{2}$ & $(1.9-3.0) \times 10^{-3} \mathrm{eV}$ & $\sqrt{\Delta m_{\mathrm{atm}}^{2}}$ & $(4.4-5.5) \times 10^{-2} \mathrm{eV}$ \\
$\Delta m_{\odot}^{2}$ & $\left(8.0_{-0.3}^{+0.4}\right) \times 10^{-5} \mathrm{eV}$ & $\sqrt{\Delta m_{\odot}^{2}}$ & $(8.9 \pm 0.3) \times 10^{-3} \mathrm{eV}$ \\
$\sqrt{\Delta m_{\odot}^{2} / \Delta m_{\mathrm{atm}}^{2}}$ & $0.16-0.20$ & & \\
\hline \hline & & & \\
\hline
\end{tabular}

The covariance matrix $V_{I J}$ determines the principal axes $Y_{i}=c_{i J} X_{J}$, with the covariance matrix $V_{i j}$ being diagonal when the basis $\left\{X_{I}\right\}$ is changed to $\left\{Y_{i}\right\}$. Since $V_{i j}$ is diagonal the observables $\left\{Y_{i}\right\}$ are independent at least up to second order. If the distributions of the $Y_{i}$ 's were completely independent, and Gaussian, then the quantity $\chi^{2}$ would follow the chi-square distribution for random measurements of $\left\{Y_{i}=c_{i J} X_{J}\right\}$. This distribution has probability density

$$
\frac{d P}{d \chi^{2}}=2^{N / 2} \Gamma(N / 2) \chi^{N-2} e^{-\chi^{2} / 2},
$$

where $N$ is the number of independent Gaussian random variables entering (45). This distribution has a mean of $N$ and a standard deviation of $\sqrt{2 N}$. Thus the prediction $\chi^{2} \approx$ $N(1 \pm \sqrt{2 / N})$ is very sharp when a large number of observables are predicted by the landscape. Note that too large a value of $\chi^{2}$ indicates that the observed flavor parameters are atypical of what is expected from the landscape distributions, while too small a value of $\chi^{2}$ indicates that the correct underlying theory has less randomness than is exhibited by the landscape in question. Therefore, even though we can measure only one value for each observable, and hence measure only one value of $\chi^{2}$, the chi-square statistic can still be a powerful tool in testing landscape theories.

Our $S^{1}$ model predicts distributions for nine flavor parameters in the quark sector, but it contains $n_{F}=2$ free parameters $\left(g_{\text {eff }}\right.$ and $\left.d / L\right)$ that can be fixed by hand and tuned ${ }^{10}$ to fit two out of nine of the $\hat{Y}_{i}$ 's, rendering two terms in $\sum_{i}\left(\hat{Y}_{i}-\left\langle Y_{i}\right\rangle\right)^{2} V_{i i}^{-1}$ to vanish. Thus we have $N=$ $9-n_{F}=7$ independent degrees of freedom. Therefore if the distributions describing the $Y_{i}$ were Gaussian, we would expect $\chi^{2} \approx 7 \pm 4$. In fact the $S^{1}$ model does not predict Gaussian distributions for any of the flavor parameters. However, Fig. 9 shows that the distribution of $\chi^{2}(45)$ using data generated by the $S^{1}$ model looks quite similar to the corresponding chi-square distribution, which has $N=$ 9. Furthermore, the central limit theorem guarantees that as $N$ is increased the distribution of $\chi^{2}$ approaches a normal distribution with a mean $N$ and a variance that grows in proportion to $N$. $N$ can be as large as 20 for the observable

\footnotetext{
${ }^{10}$ In fact the free parameters $g_{\text {eff }}$ and $d / L$ have not been fully exploited, since we have not performed a maximum likelihood analysis to determine the values that minimize $\chi^{2}$ in (45). However, the parameter values we use have been chosen to qualitatively fit our expectations for the measured values of $\left\{X_{I}\right\}$.
} 

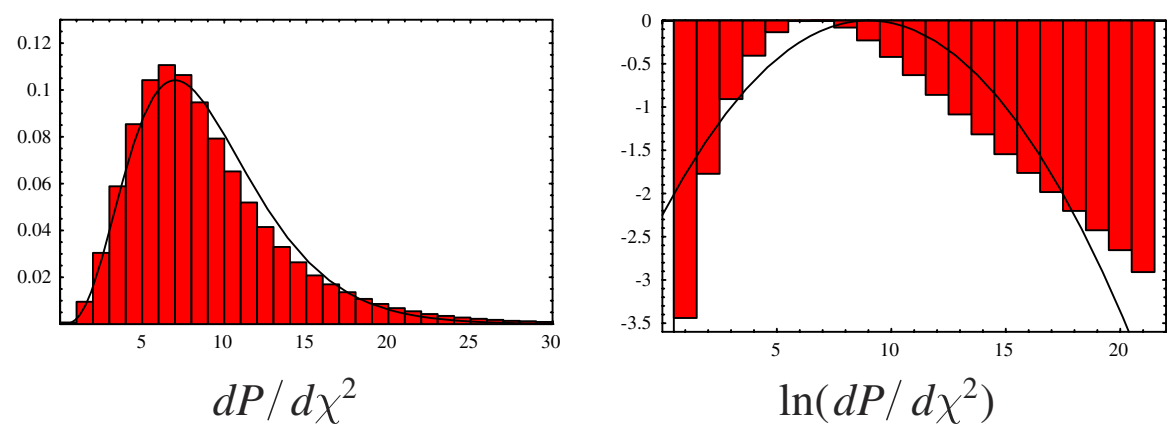

FIG. 9 (color online). The left panel displays the probability distribution of $\chi^{2}$ obtained numerically (histogram) using data generated by the $S^{1}$ model, along with the actual chi-square distribution for $N=9$. The right panel displays the logarithm of the numerical distribution of $\chi^{2}$ (histogram) and a normal distribution with a mean $N=9$ and standard deviation $\sqrt{2 N}=\sqrt{18}$.

flavor parameters of the standard model, assuming a landscape with at least 20 scanning parameters.

We calculate the chi-square statistic for the nine $C P$-conserving quark-sector flavor parameters using the $S^{1}$ model to calculate $\left\langle X_{I}\right\rangle$ and $V_{I J}$, and using Table I for $\hat{X}_{I}$. This gives $\chi^{2} \approx 6$, which agrees very well with the prediction $\chi^{2} \approx 7 \pm 4$. That the obtained value is neither too large nor too small indicates that the observed flavor parameters are typical and that they exhibit the expected level of statistical fluctuation in this landscape. The same analysis can be carried out for the $S^{1}$ model after a hypothetical environmental weight factor favoring a large top mass has been imposed. Using only $S^{1}$ model data that pass the $t$-cut (43), we find $\chi^{2} \approx 8$, which also agrees well with the prediction. Imposing the $t$-cut increases the value of $\chi^{2}$, but one should keep in mind that the parameters $g_{\text {eff }}$ and $d / L$ were chosen to fit the observed flavor parameters before imposing the $t$-cut, without respect for the ensemble that passes the $t$-cut.

\section{B. The p-value statistic}

The chi-square statistic has a disadvantage as well. Although the central limit theorem guarantees that for large $N$ that the distribution of $\chi^{2}$ approaches a normal distribution, regardless of most of the details of the actual distributions of the $\left\{X_{I}\right\}, N$ is not more than 20 for the application to flavor parameters. Moreover, some of the distributions of the flavor observables have sharp cutoffs, such that the probability to obtain a large value of $\left(\hat{X}_{I}-\right.$ $\left.\left\langle X_{I}\right\rangle\right)^{2}$ may be much smaller than what is expected from a normal distribution with a mean $\left\langle X_{I}\right\rangle$ and a variance $V_{I I}$. The chi-square statistic does not completely account for such a situation.

On the other hand, the p-value statistic is capable of handling non-Gaussian distribution functions. The p-value of a single flavor parameter $X_{I}$ is simply the fraction of the distribution that is more atypical than the measured value $\hat{X}_{I}$. Specifically, we may consider the p-value to be the fraction of the distribution that is farther from the median $\bar{X}_{I}$ than is $\hat{X}_{I}$. If we approximate the probability density as continuous with profile $f\left(X_{I}\right)$, then the p-value $p_{I}$ is

$$
\begin{array}{ll}
p_{I}\left(\hat{X}_{I}\right)=\int_{-\infty}^{\hat{X}_{I}} 2 f\left(X_{I}\right) d X_{I} & \text { for } \hat{X}_{I}<\bar{X}_{I}, \\
p_{I}\left(\hat{X}_{I}\right)=\int_{\hat{X}_{I}}^{\infty} 2 f\left(X_{I}\right) d X_{I} & \text { for } \hat{X}_{I}>\bar{X}_{I} .
\end{array}
$$

Note that like $\chi^{2}, p_{I}$ is a random variable for every independent measurement $\hat{X}_{I}$. However, whereas $\chi^{2}$ is distributed according to the chi-square distribution, $p_{I}$ is distributed uniformly between zero and one. The p-value is also the probability that the Kolmogorov-Smirnov statistic (the value of the $D$ function) of one sampling of the variable $X_{I}$ could have been larger than what is calculated from $\hat{X}_{I}$. Hence a measurement giving $\mathrm{p}$-value $p_{I}$ excludes the corresponding theory at a confidence level of $\max \left\{p_{I}, 1-p_{I}\right\} \times 100 \% .^{11}$

Reference [15] used the p-value statistic to test the hypothesis of neutrino anarchy. There, the probability distribution of the lepton sector flavor parameters is factorized into those of mass eigenvalues, of each individual mixing angle, and of the $C P$ phase [15]. Then the p-value statistic can be applied separately to each of the three mixing angles. In the case where the statistical variables $X_{I}$ are independent of each other, $\hat{k} \equiv \prod_{I} p_{I}\left(\hat{X}_{I}\right)$ represents the fraction of the distribution of $\left\{X_{I}\right\}$ for which each element $X_{I}$ is more atypical than the measured value $\hat{X}_{I}$. The probability that $k \equiv \prod_{I} p_{I}\left(X_{I}\right)$ could have been smaller than $\hat{k}$ is given by (e.g. [15])

$$
p=\int^{k \leq \hat{k}} \prod_{I} d p_{I}=\hat{k} \sum_{a=0}^{N-1} \frac{1}{a !}(-\ln \hat{k})^{a} .
$$

Here $a$ is simply a summation index used to simplify the last expression, and $N$ is the number of degrees of freedom

\footnotetext{
${ }^{11} \mathrm{P}$-values very near zero indicate that the measured values $\left\{\hat{X}_{I}\right\}$ are collectively atypical of their predicted distributions; $p$-values very near unity indicate that the measured values do not exhibit the randomness expected from the predicted distributions.
} 
in $\left\{X_{I}\right\}$. For a random measurement of a set of independent variables $\left\{X_{I}\right\}$, the p-value calculated through (52) is distributed uniformly from zero to one. Thus a random measurement giving $\mathrm{p}$-value $p$ can exclude a landscape theory with a confidence level $\max \{p, 1-p\} \times 100 \%$. If we treat the nine $C P$-conserving flavor observables in the quark sector as if they were independent, then the data $\hat{X}_{I}$ and the hypothetical distributions $d P\left(X_{I}\right) / d X_{I}$ of the $S^{1}$ model can be used to calculate the p-value through (52). Here, $N=7$ is used in (52) because the values of $g_{\text {eff }}$ and $d / L$ can be tuned to bring two of the $X_{I}$ to be the same as their medians. We find $p \simeq 0.66$, suggesting a good fit with observation.

In fact, however, the statistical variables $\left\{X_{I}\right\}$ are not independent. To the best of our knowledge, there is no standard definition of the p-value statistic for correlated multivariable distributions. One possibility to account for correlation is to calculate $\hat{k}$ by actually counting the fraction of randomly generated parameter sets $\left\{X_{I}\right\}$ that have each $X_{I}$ more atypical than that of the measured set $\left\{\hat{X}_{I}\right\}$. This is as opposed to using $\hat{k}=\prod_{I} p_{I}\left(\hat{X}_{I}\right)$, which gives this fraction in the absence of correlations. In a sample of $5 \times$ $10^{6}$ data sets generated using the numerical simulation of the $S^{1}$ model, we find 52 such sets, giving $\hat{k}=2^{9} \times\left(\frac{52}{5 \times 10^{6}}\right)$ and hence $p \simeq 0.73 \pm 0.03$. The uncertainty is obtained by assuming the variance in counting $N_{\text {atyp }}$ atypical data points is of the order of $N_{\text {atyp }}$. Yet another attempt at a "p-valuelike" measure of the goodness-of-fit may be to judge atypicality by using in (51) the distributions of the principal axes $Y_{i}$, their measured values $\hat{Y}_{i}$, and their medians $\bar{Y}_{i}=c_{i J} \bar{X}_{J}$-as opposed to using $X_{I}, \hat{X}_{I}$, and $\bar{X}_{I}$ - since the statistical variables $Y_{i}$ do not have correlations, at least up to second order. The fraction of $\left\{Y_{i}\right\}$ more atypical than the measured set $\left\{\hat{Y}_{i}\right\}$ can be calculated using $\hat{k}=\prod_{i} p_{i}\left(\hat{Y}_{i}\right)$; using (52) we find this gives $p \simeq 0.42$. If there were truly no correlations among the $Y_{i}$ this would give the same result as calculating $\hat{k}$ by actually counting the fraction of randomly generated parameter sets $\left\{Y_{i}\right\}$ that have each $Y_{i}$ more atypical than that of the measured set $\left\{\hat{Y}_{i}\right\}$. However, in our ensemble of $5 \times 10^{6}$ data sets we find just one for which each element is more atypical than its corresponding measured value, giving $p \simeq 0.19_{-0.19}^{+0.07}$.

\section{GEOMETRY DEPENDENCE}

In Sec. III we introduced and analyzed a Gaussian landscape based on a single extra dimension and found that it could provide the hierarchy, pairing, and generation structures of the observed quark sector. However, the number and geometry of extra dimensions need not correspond to the $S^{1}$ Gaussian landscape. Therefore, we initiate a study into how the compactification geometry of extra dimensions affects the probability distributions of observables. We find that the qualitative results of Sec. III can be achieved by Gaussian landscapes in other geometries of extra dimensions and that the distribution functions of flavor observables are largely insensitive to the details of these geometries. However, the behavior of the distribution functions of mass eigenvalues at large values is affected by the number of extra dimensions. In Sec. VII, we argue that the geometries used in Gaussian landscapes are related to base manifolds of torus-fibered geometries used in the compactification of string theory.

\section{A. Gaussian landscapes on $T^{2}$ and $S^{2}$}

To investigate the robustness of the Gaussian landscape on $S^{1}$, we study other geometries. We first look at the simplest extension to more than one dimension, $T^{2}=S^{1} \times$ $S^{1}$, and focus on a "square torus" where the two periods of the torus are both $L$, and the directions of the two $S^{1}$ are orthogonal. We assume that each of the quarks and the Higgs have rotation-symmetric Gaussian wave functions of the form

$$
\varphi\left(\vec{y} ; \vec{y}_{0}\right) \propto e^{-\left(\left|\vec{y}-\vec{y}_{0}\right|^{2} / 2 d^{2}\right)},
$$

where the center coordinates $\vec{y}_{0}$ of each particle are randomly scanned over the internal space $T^{2}$. The up-type and down-type Yukawa matrices are calculated by the overlap integration (22), which is naturally generalized to integration on $T^{2}$. We defer to Sec. VII a discussion of the extent to which these assumptions result from a dynamical field theory on extra-dimensional spacetime, and for the moment focus on the phenomenology of this landscape.

Figure 10 shows the distribution of Yukawa matrix elements for a numerical simulation of this model. As in the $S^{1}$ model, the only parameters relevant to these distri-
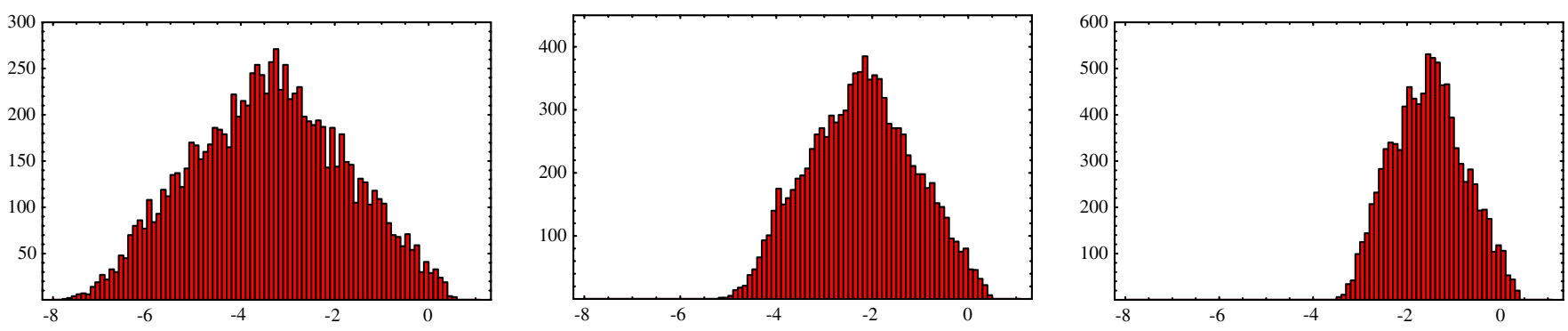

FIG. 10 (color online). Distributions of $10^{4}$ Yukawa matrix elements, generated from the Gaussian landscape on $T^{2}$. From left to right the panels correspond to $\left(d / L, g_{\text {eff }}\right)=(0.1,1),(0.12,1)$ and $(0.14,1)$. 
butions are $g_{\text {eff }}=g /\left(M_{6} L\right)$ and $d / L$, where now $M_{6}$ is the cutoff scale of the effective theory in $5+1$ dimensions. Furthermore, a larger hierarchy is generated when the wave functions are more localized, i.e. when $d / L$ is smaller. The key difference from $S^{1}$ is that the distribution of Yukawa matrix elements is no longer scale invariant. Instead, on $T^{2}$ the probability density for the largest and the smallest matrix elements is depleted. With regard to the Yukawa eigenvalues and mixing angles, we find that a hierarchical pattern of Yukawa eigenvalues is generated (Fig. 11) and that the distributions of mixing angles are peaked at $\theta_{i j}=$ 0 but not at $\theta_{i j}=\pi / 2$ (Fig. 12). Thus the flavor structure of the quark sector follows from the Gaussian landscape on $T^{2}$. Despite the apparent difference between the distribution of the Yukawa matrix elements of the two landscapes (comparing Figs. 4 and 10), we see that the distribution of masses and mixing angles are roughly the same when we compare Figs. 11 and 12 to Figs. 7 and 8. In particular, these distributions all come with a width of about an order of magnitude, and the differences between the distributions from the two toy landscapes is not, statistically, very significant compared to this width. This demonstrates that the flavor structure in the quark sector is a robust feature of Gaussian landscapes.

The biggest difference between the distribution of observables in the two toy landscapes is a more left/right symmetric probability distribution for $\log _{10} \lambda_{b, t}$ and $\log _{10} \lambda_{s, c}$ on $T^{2}$. This is a consequence of the difference in the distribution of individual Yukawa matrix elements, Fig. 10 vs Fig. 4. The depleted probability density of the largest Yukawa matrix elements in the Gaussian landscape on $T^{2}$ results in reduced probability for largest values of $\lambda_{b, t}$ and $\lambda_{s, c}$. The distribution of $\lambda_{b, t}$ becomes much broader and less peaked at the largest possible value, $\lambda_{\max }$. This allows the bottom Yukawa coupling to become as small as its measured value rather easily, while still accommodating the measured value of the top-Yukawa coupling.

The effects of a possible environmental selection for a large top-Yukawa coupling are studied in Fig. 11 and in
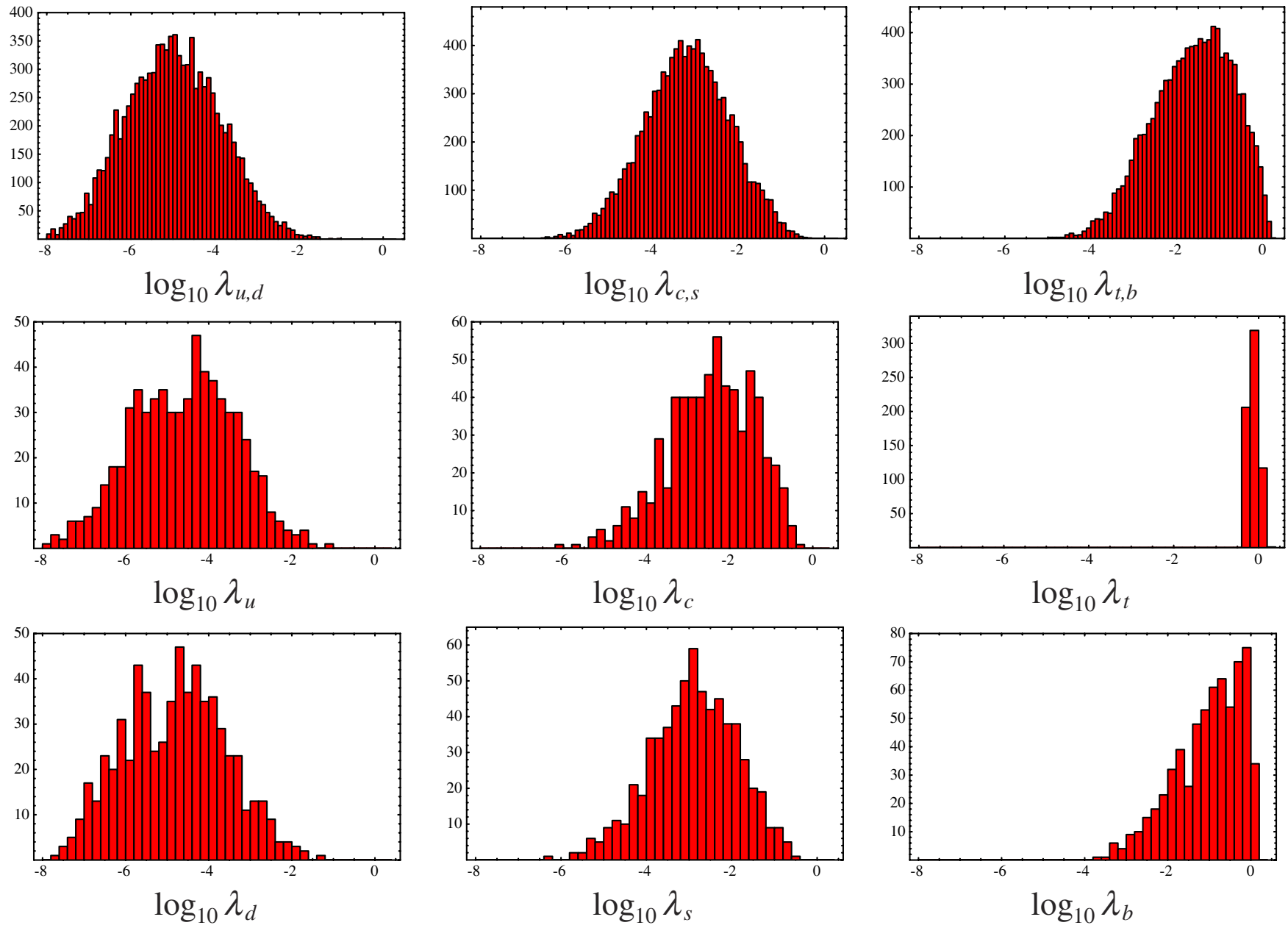

FIG. 11 (color online). Distributions of Yukawa eigenvalues in the Gaussian landscape on $T^{2}$, based on a numerical simulation with $\left(d / L, g_{\text {eff }}\right)=(0.1,0.4)$. The first row shows the distribution of the three eigenvalues of the up (and down) sector. The second and third rows display the eigenvalues of the roughly $6 \%$ of matrices that survive the $t$-cut of Sec. IIIC. 

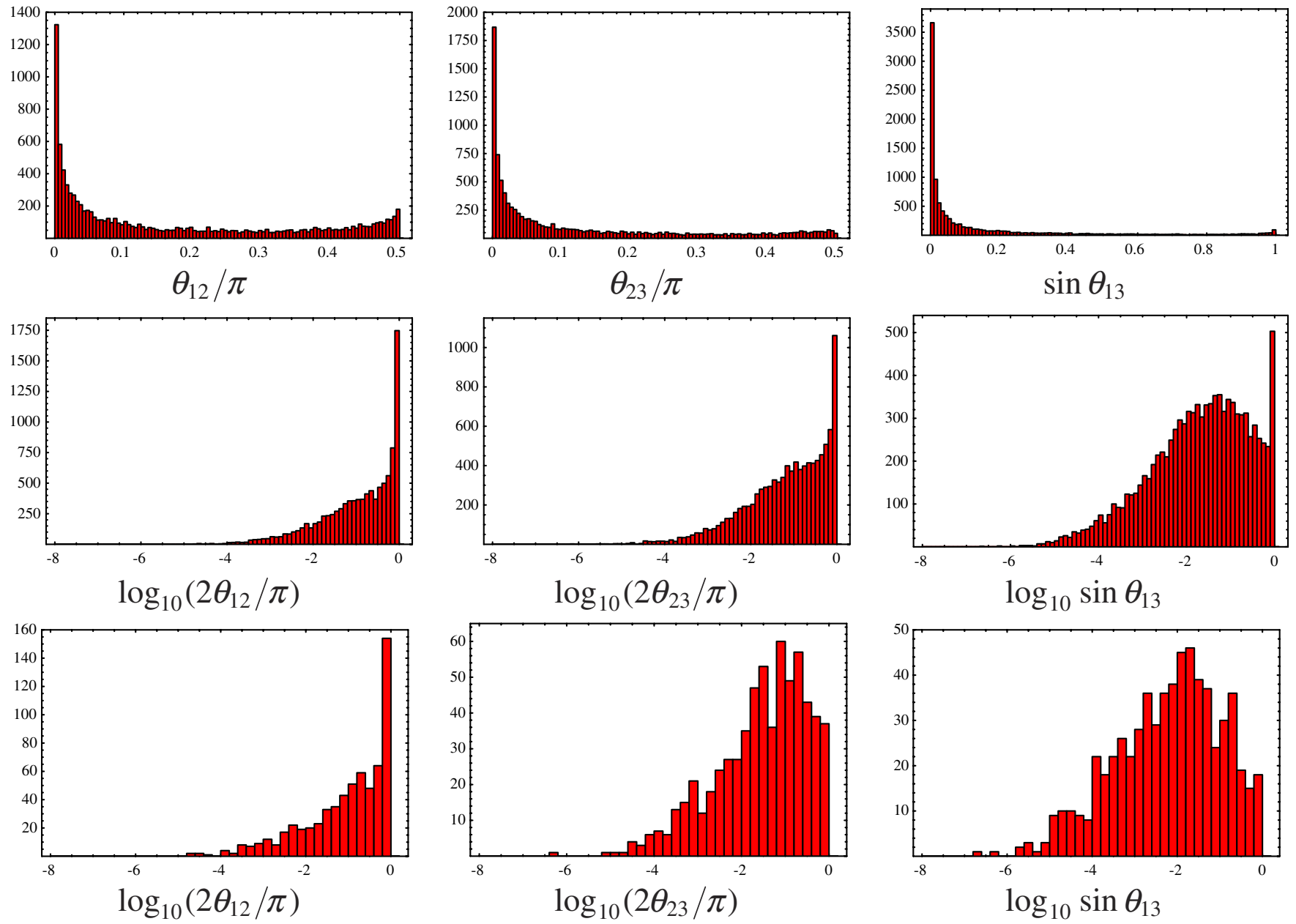

FIG. 12 (color online). Distributions of CKM mixing angles in the Gaussian landscape on $T^{2}$, based on a numerical simulation with $\left(d / L, g_{\text {eff }}\right)=(0.1,0.4)$. The bottom row displays the mixing angles of the roughly $6 \%$ of matrices that survive the $t$-cut of Sec. III C.

Fig. 12, using the cut condition (43) as a crude approximation to the environmental selection effect. The distributions of the mixing angles $\theta_{23}$ and $\theta_{13}$ are shifted toward smaller angles, just as on $S^{1}$. Since the cut condition is in favor of a larger hierarchy between the lighter quarks and the heaviest quarks, smaller $\theta_{23}$ and $\theta_{13}$ are natural consequences. The inequality $\left.\left\langle\lambda_{c}\right\rangle\right\rangle\left\langle\lambda_{s}\right\rangle$ also follows from the cut, just like in Sec. III. The most important difference between the two landscapes may be in the distribution of the bottom Yukawa coupling after the cut is imposed. It was rare that $\lambda_{b}$ be less than $10^{-2}$ in the lower-right distribution of Fig. 7, but a significant fraction is below $10^{-2}$ in Fig. 11. Therefore the observed hierarchy $\lambda_{t} / \lambda_{b}$ may be understood within the context of a Gaussian landscape with an appropriately chosen geometry, especially when there is environmental selection for a large topYukawa coupling.

For comparison, we also perform a numerical study of a Gaussian landscape defined on $S^{2}$. As before, the quarks and Higgs are represented by localized wave functions with width $d$. Specifically, on $S^{2}$ a wave function centered at $\theta=0$ is given by ${ }^{12}$

$$
\varphi(\theta, \phi) \propto e^{-\left(\theta^{2} / 2(d / R)^{2}\right)} .
$$

Of course, to generate an ensemble of Yukawa matrices the central coordinates of each wave function are scanned independently and uniformly over the geometry $S^{2}$. Note that like the previously described landscapes, the Gaussian landscape on $S^{2}$ is characterized by two free parameters, $d / R$ and $g_{\text {eff }}=g /\left(\sqrt{\pi} M_{6} R\right)$.

Figure 13 shows the distribution of Yukawa matrix elements for this model. Note that the overall shapes of the distributions are remarkably similar to those in Fig. 10, which correspond to $T^{2}$. Figure 14 displays the distributions of the three mass eigenvalues for the Gaussian landscapes on $S^{2}$ and $T^{2}$. These distributions are quite similar between $S^{2}$ and $T^{2}$. We find that the other phenomenologi-

\footnotetext{
${ }^{12}$ Although this wave function is not smooth at $\theta=\pi$, this is not of present concern. That is, the purpose of this numerical simulation is not to determine the distribution precisely but to study its qualitative aspects.
} 

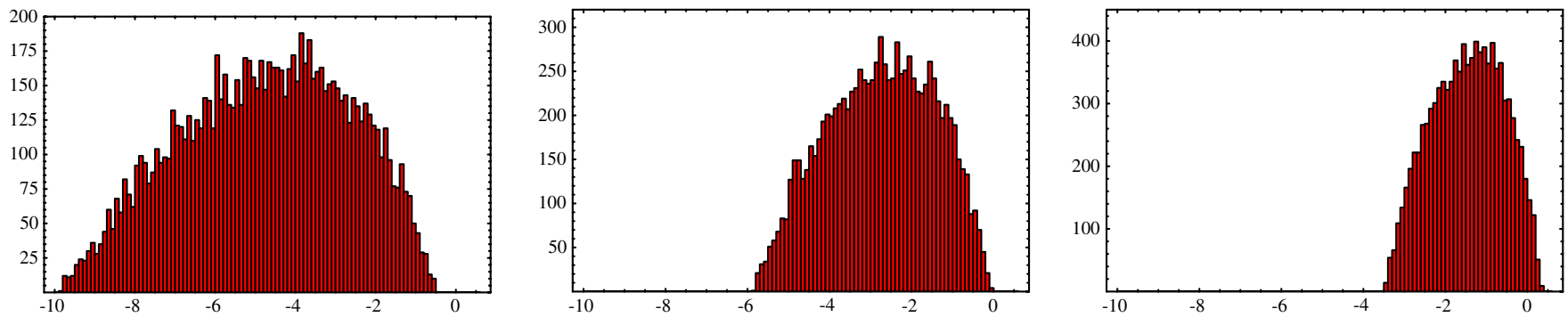

FIG. 13 (color online). Distributions of $10^{4}$ Yukawa matrix elements, generated from the Gaussian landscape on $S^{2}$. From left to right the panels correspond to $\left(d / R, g_{\text {eff }}\right)=(0.4,1),(0.5,1)$ and $(0.6,1)$.
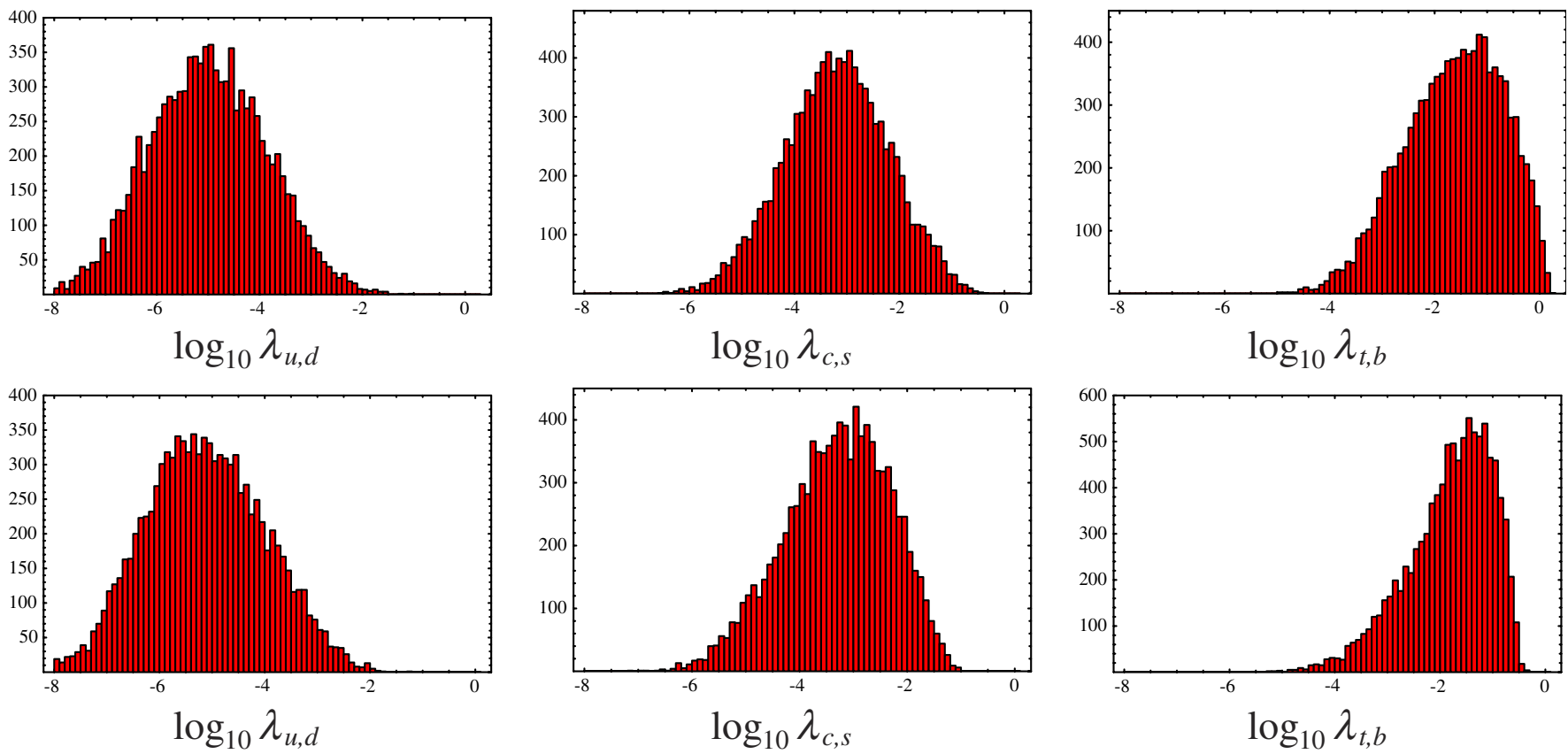

FIG. 14 (color online). Comparison between the distributions of Yukawa eigenvalues from the Gaussian landscape on $T^{2}$ with $\left(d / L, g_{\text {eff }}\right)=(0.1,0.4)$ (top row) and the Gaussian landscape on $S^{2}$ with $\left(d / R, g_{\text {eff }}\right)=(0.45,0.4)$ (bottom row).

cal aspects of the Gaussian landscape, discussed above with respect to the geometry $T^{2}$, hold true on $S^{2}$ as well.

\section{B. Dependence on dimensionality and geometry independence}

Having seen that the hierarchical mass eigenvalues and the generation structure of the quark sector are robust predictions of Gaussian landscapes, the next subject of interest is to understand to what extent the details of distribution functions depend on the internal geometry. Already we have seen slight differences between the Gaussian landscape on $S^{1}$ and the landscapes defined on $T^{2}$ and $S^{2}$, yet remarkable similarity between the distributions coming from $T^{2}$ and $S^{2}$. We would like to understand where these differences and similarities come from, without having to run simulations on all possible choices of internal geometry. To do this, we recall the analysis of Secs. III A and III B, where the distributions of flavor observables on $S^{1}$ were understood analytically within the context of the AFS approximation. As we show in this section, this analysis can be generalized to Gaussian landscapes on any geometry of extra dimensions. ${ }^{13}$

Let us consider a $D$-dimensional internal space with local coordinates $\vec{y}$. Then in the limit where we can ignore the finite size of the internal space the Yukawa coupling matrix is given by

$$
\lambda_{i j}^{u} \propto e^{-(1 / 3 d)\left(\left|\vec{a}_{i}\right|^{2}+\left|\vec{b}_{j}\right|^{2}-\vec{a}_{i} \cdot \vec{b}_{j}\right)},
$$

which replaces $\lambda_{i j}^{u}$ in (24) for the Gaussian landscape on

\footnotetext{
${ }^{13}$ There is also a practical motivation for this approach. The numerical integration time involved with performing overlap integrals on extra dimensions grows very large as the number of extra dimensions is increased; meanwhile generating ensembles of flavor observables involves performing large numbers of these integrals.
} 
$S^{1}$. Here $\vec{a}_{i}$ and $\vec{b}_{j}$ are the central coordinates of the quark wave functions, relative to that of the Higgs boson. The Yukawa matrix again has the AFS form (25), with suppression factors

$$
\epsilon_{j}^{q}=e^{-\left(\left|\vec{b}_{j}\right|^{2} / 3 d^{2}\right)}, \quad \epsilon_{i}^{\bar{u}}=e^{-\left(\left|\vec{a}_{i}\right|^{2} / 3 d^{2}\right)},
$$

replacing (26). In the extra factor $g_{i j}=e^{\vec{a}_{i} \cdot \vec{b}_{j} / 3 d^{2}}$ (which is not necessarily of order unity) the vectors $\vec{a}_{i}$ and $\vec{b}_{j}$ are sometimes unaligned, sometimes parallel, and sometimes antiparallel. This generates a random coefficient to each Yukawa coupling that is statistically neutral in the AFS approximation. We note again that this analysis is valid only when the compactness of the internal space is unimportant and the local geometry can be approximated as a flat $D$-dimensional space. This is equivalent to focusing on only the largest Yukawa matrix elements.

Ignoring the statistically neutral factor $e^{\vec{a}_{i} \cdot \vec{b}_{j} / 3 d^{2}}$, the Yukawa matrix elements are roughly

$$
\lambda \sim e^{-\left(|\overrightarrow{\mathbf{r}}|^{2} / 3 d^{2}\right)}, \quad-\ln \lambda=\frac{|\overrightarrow{\mathbf{r}}|^{2}}{3 d^{2}},
$$

with $\overrightarrow{\mathbf{r}}=(\vec{a}, \vec{b})$ scanning a $2 D$-dimensional space. The natural probability measure is

$$
d P \propto \frac{d^{2 D} \overrightarrow{\mathbf{r}}}{L^{2 D}} \propto \frac{|\overrightarrow{\mathbf{r}}|^{2 D-2} d|\overrightarrow{\mathbf{r}}|^{2}}{L^{2 D}} \propto\left(\frac{d}{L}\right)^{2 D}(-\ln \lambda)^{D-1} d|\ln \lambda|,
$$

where $L$ is the typical size of the extra dimensions. Indeed, the distribution of the Gaussian landscape on $S^{1}(D-1=$ 0 ) is flat, c.f. Fig. 4, and those of the Gaussian landscapes on $T^{2}$ and $S^{2}(D-1=1)$ are linear in $|\ln \lambda|$ at their upper end. ${ }^{14}$ These results are independent of the value of $d / L$. The logarithmic range of the distribution of Yukawa couplings scales as $(L / d)^{2}$.

The distribution of the AFS suppression factors, $\epsilon_{j}^{q}$, can be obtained as in Sec. III. In more than one dimension the measure (31) is generalized to

$$
d P(b) \sim \frac{d^{D} b}{L^{D}} \sim L^{2-D} \frac{d V(b)}{d b^{2}} \frac{d b^{2}}{L^{2}},
$$

where $b \equiv|\vec{b}|$ and $V(b)$ is the volume enclosed within a distance $b$ from a given point. Using (56), it can be converted into a distribution of the AFS suppression factors,

$$
d P(y)=f(y) d y, \quad f(y)=\left.\frac{b_{\max }^{2}}{V_{\mathrm{tot}}} \frac{d V(b)}{d b^{2}}\right|_{b=b_{\max } \sqrt{y}} .
$$

Here, $\ln \epsilon$ is normalized by $\Delta \ln \epsilon=-\frac{1}{3}\left(b_{\max } / d\right)^{2}$, so that

\footnotetext{
${ }^{14}$ The distributions on $S^{2}$ are not as precisely linear for large matrix elements as those on $T^{2}$. This can be understood in terms of geometry: $S^{2}$ has positive curvature while $T^{2}$ is flat.
}

$y \equiv \ln \epsilon / \Delta \ln \epsilon=\left(b / b_{\max }\right)^{2}$ ranges from zero to one. For example, $b_{\max }=L / 2$ for $S^{1}, b_{\max }=L / \sqrt{2}$ for $T^{2}$ and $b_{\max }=\pi R$ for $S^{2}$. The ratio $\left(b_{\max } / d\right)^{2}$ determines the overall logarithmic range of hierarchy, and the volume distribution function $f(y)$ controls the shape of the distributions within $y \in[0,1]$. It is straightforward to find $f(y)$ for a given geometry of extra dimensions; for example, for $S^{1}, S^{2}$, and $S^{3}$,

$$
\begin{gathered}
f_{S^{1}}(y)=\frac{1}{2 \sqrt{y}}, \quad f_{S^{2}}(y)=\frac{\pi}{4} \frac{\sin (\pi \sqrt{y})}{\sqrt{y}}, \\
f_{S^{3}}(y)=\frac{\sin ^{2}(\pi \sqrt{y})}{\sqrt{y}},
\end{gathered}
$$

and for the "square torus" $T^{2}$,

$$
f_{T^{2}}(y)=\frac{\pi}{2}-2 \arcsin \left(\sqrt{1-\frac{1}{2 y}}\right) \Theta(y-1 / 2) .
$$

These volume distribution functions are displayed in the first row of Fig. 15.

The distribution of the smallest, middle, or largest AFS suppression factor is obtained by integrating the other two variables out of the probability distribution

$$
\begin{aligned}
d P\left(y_{1}, y_{2}, y_{3}\right)= & 3 ! f\left(y_{1}\right) f\left(y_{2}\right) f\left(y_{3}\right) \Theta\left(y_{1}-y_{2}\right) \\
& \times \Theta\left(y_{2}-y_{3}\right) d y_{1} d y_{2} d y_{3},
\end{aligned}
$$

where $y_{i} \equiv \ln \epsilon_{i} / \Delta \ln \epsilon$ and we remind that in this notation $y_{1}>y_{2}>y_{3}$. The AFS suppression factors $y_{i}$ for $S^{1,2,3}$ are displayed in the second row of Fig. 15. The mass eigenvalues $\lambda_{i}^{u(d)}$ are approximated by $\epsilon_{i}^{q} \epsilon_{i}^{\bar{u}(\bar{d})}$, and hence the distribution of $z_{i} \equiv \ln \left(\lambda_{i}^{u(d)} / \lambda_{\max }\right) / \Delta \ln \epsilon$ is given by a convolution of the distribution function of $y_{i}=$ $\ln \epsilon_{i} / \Delta \ln \epsilon$ (third row of Fig. 15). The diagonalization matrices for the up and down sectors are determined by three diagonalization angles, which are approximately equal to $\epsilon_{i} / \epsilon_{j}, i<j$. Thus, the distribution of $\Delta_{i j} \equiv$ $\ln \left(\epsilon_{i} / \epsilon_{j}\right) / \Delta \ln \epsilon=y_{i}-y_{j}$ is obtained by integrating the variables $y_{1,2,3}$ out of (63) while keeping the distance $\Delta_{i j}=$ $y_{i}-y_{j}$ (fourth row of Fig. 15). Finally, the CKM mixing angles are obtained from the diagonalization angles by approximating the mixing angles to be the larger one of the two $\Delta_{i j}$ coming from the up and the down sectors. These are displayed in the fifth row of Fig. 15. Note that the analytical results for the mass eigenvalue distributions (the third row of Fig. 15) capture the qualitative features of the numerical results for the Gaussian landscapes on $S^{1}, T^{2}$, and $S^{2}$ very well.

It is now possible to understand why the distributions of flavor observables are quite similar between the Gaussian landscapes on $T^{2}$ and $S^{2}$. All of these distributions are calculated using only the volume distribution function $f(y)$. Although $f(y)$ is quite different for these two geometries, as is seen in the first row of Fig. 15, a number of 

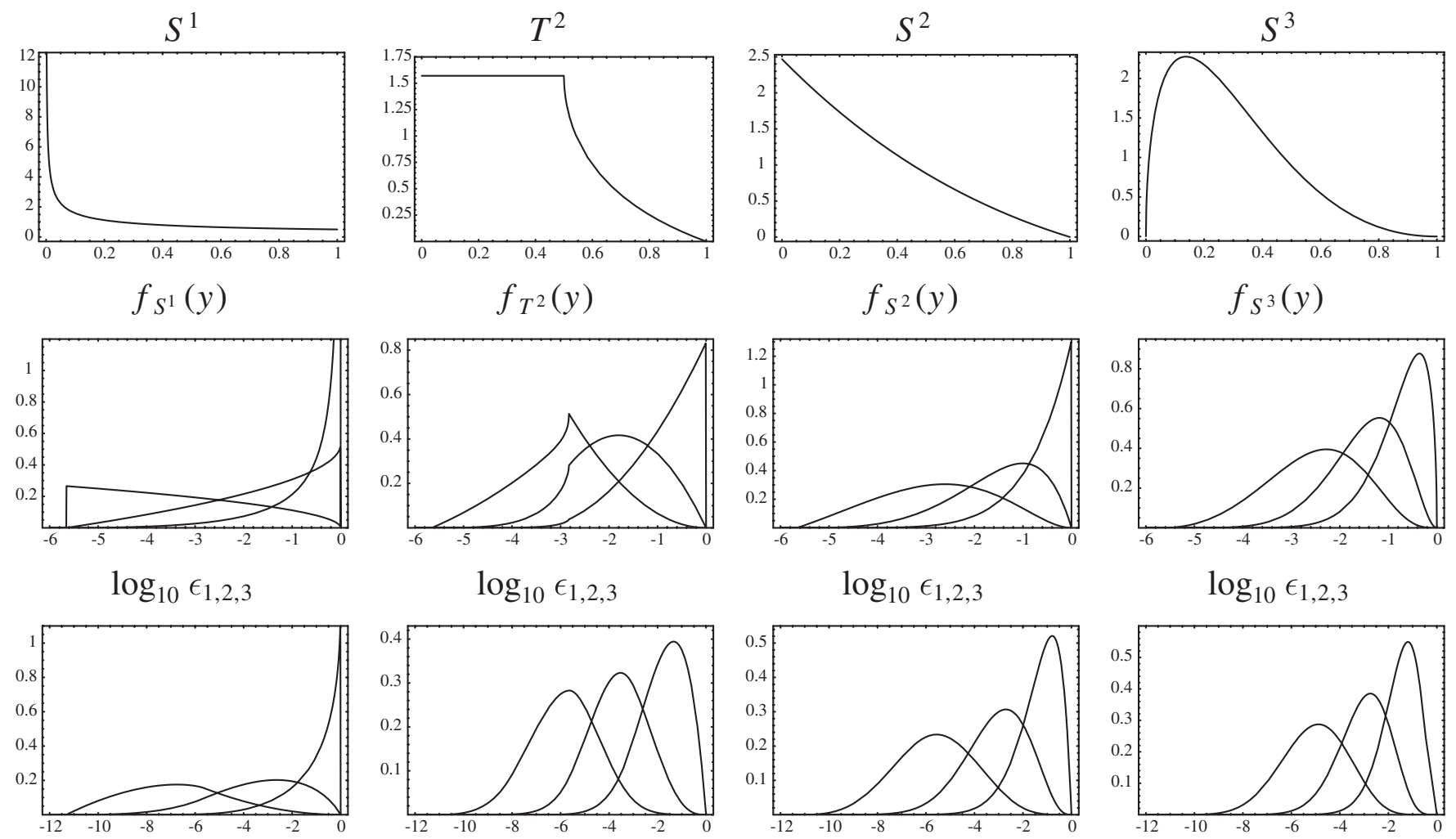

$\log _{10} \lambda_{1,2,3}$

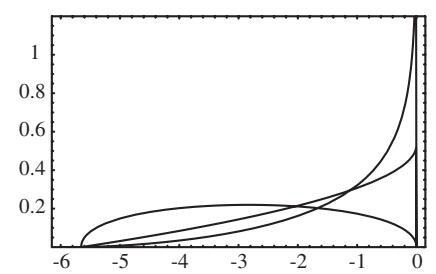

$\log _{10} \lambda_{1,2,3}$

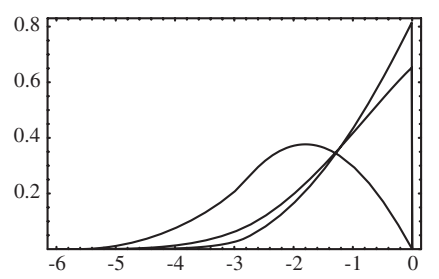

$\log _{10} \lambda_{1,2,3}$

$\log _{10} \lambda_{1,2,3}$
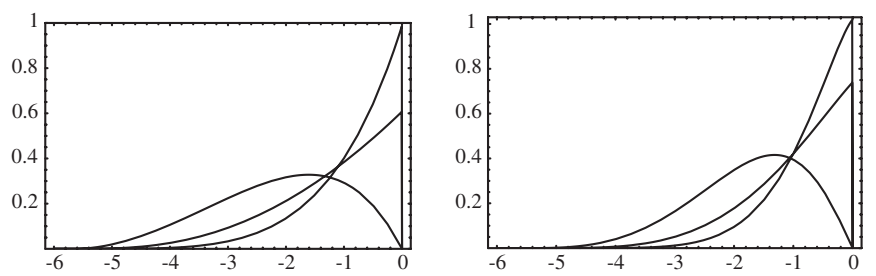

$\log _{10}\left(\epsilon_{i} / \epsilon_{j}\right)$

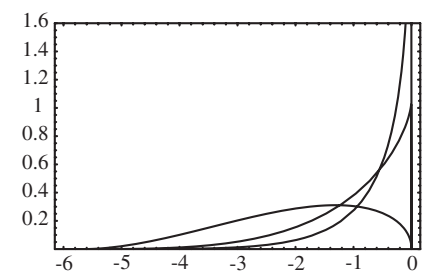

$\log _{10}\left(\epsilon_{i} / \epsilon_{j}\right)$

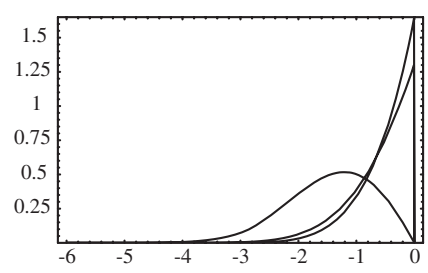

$\log _{10}\left(\epsilon_{i} / \epsilon_{j}\right)$

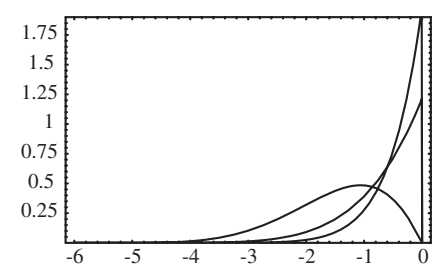

$\log _{10} \sin \theta_{12,23,13}$

$\log _{10} \sin \theta_{12,23,13}$

$\log _{10} \sin \theta_{12,23,13}$

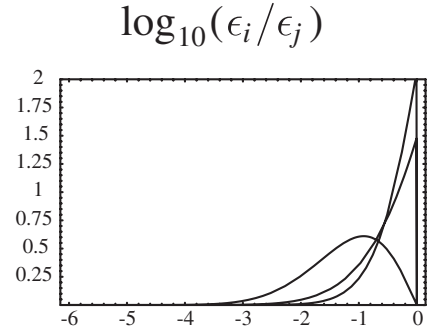

$\log _{10} \sin \theta_{12,23,13}$

FIG. 15. The volume distribution functions $f(y)$, AFS suppression factors $\log _{10} \epsilon_{1,2,3}$, the Yukawa eigenvalues $\log _{10} \lambda_{1,2,3}$, the diagonalization angles $\log _{10}\left(\epsilon_{i} / \epsilon_{j}\right)(i<j)$, and the CKM mixing angles $\log _{10} \sin \theta_{i j}$ for $S^{1}, T^{2}, S^{2}$, and $S^{3}$. In all cases the broadest mixing-angle distribution corresponds to $\theta_{13}$, while the distribution most sharply peaked at zero corresponds to $\theta_{23}$. These use approximations (36) and (38)-(40), which are not reliable for small Yukawa eigenvalues. For clear comparison the variables $y_{i}, z_{i}$, and $t_{i j}$ have been converted to observables using a common logarithmic scale, $\Delta \log _{10} \epsilon=-5.66$, that corresponds to the scale of the Gaussian landscape on $S^{1}$ with $d / L=0.08$.

consecutive integrations is required to obtain distribution functions for observable flavor parameters. For any AFS suppression factor $y_{i} \propto \ln \epsilon_{i}$, the volume distribution function $f\left(y_{i}\right)$ in (63) is not integrated, and thus singularities in the original $f\left(y_{i}\right)$ remain in the distribution of $\log _{10} \epsilon_{i}$.
Nevertheless, the difference in $f(y)$ between $T^{2}$ and $S^{2}$ is already less evident in the distributions of $\log _{10} \epsilon_{i}$ (second row of Fig. 15), because the integration of two variables out of (63) takes a certain average of $f(y)$. Meanwhile, to obtain the distribution functions of the mass eigenvalues, 
diagonalization angles, and the CKM mixing angles, each $f\left(y_{i}\right)$ is integrated at least once, and the geometrydependent information contained in $f(y)$ is further smeared out. This is why the distributions of the Yukawa eigenvalues are quite similar between $T^{2}$ and $S^{2}$ in Figs. 14 and 15. Since the distributions of CKM mixing angles involve a greater number of integrations, these distributions are even less sensitive to the underlying geometry.

The analytical discussion so far explains why the distributions of flavor observables are similar for the Gaussian landscapes on $T^{2}$ and $S^{2}$, but it remains unseen why the distributions of mass eigenvalues of the $S^{1}$ Gaussian landscape are somewhat different from those of $T^{2}$ and $S^{2}$. Therefore, we now focus on how the number of dimensions in the internal geometry affects the distributions of flavor observables. As we have already seen in the discussion surrounding (58), the number of extra dimensions directly affects the shape of the volume distribution function. Specifically, we find $f(y) \propto y^{D / 2-1}$ for $y$ greater than but near zero. Thus for very small $y_{i}$,

$$
\begin{gathered}
d P\left(y_{1}\right) \propto y_{1}^{3 D / 2-1} d y_{1}, \quad d P\left(y_{2}\right) \propto y_{2}^{D-1} d y_{2}, \\
d P\left(y_{3}\right) \propto y_{3}^{D / 2-1} d y_{3} .
\end{gathered}
$$

Distribution functions of the masses and mixing angles can be obtained by using the approximations (36) and (38)(40), analogous to the analysis earlier in this section. In the limit of large eigenvalues and large mixing angles these distribution functions behave as

$$
\begin{array}{rlrl}
\frac{d P\left(z_{1}\right)}{d z_{1}} \propto z_{1}^{3 D-1}, & & \frac{d P\left(z_{2}\right)}{d z_{2}} \propto z_{2}^{2 D-1}, \\
\frac{d P\left(z_{3}\right)}{d z_{3}} \propto z_{3}^{D-1}, & \frac{d P\left(t_{12}\right)}{d t_{12}} \neq 0, \\
\frac{d P\left(t_{23}\right)}{d t_{23}} \neq 0, & \frac{d P\left(t_{13}\right)}{d t_{13}} \propto t_{13},
\end{array}
$$

where again $z_{i} \equiv \ln \left(\lambda_{i} / \lambda_{\max }\right) / \Delta \ln \epsilon \quad$ and $\quad t_{i j} \equiv$ $\ln \sin \theta_{i j} / \Delta \ln \epsilon$. Note that the behavior of the distribution functions of the mixing angles does not depend on the number of dimensions. The $D$ dependence above is qualitatively confirmed in Fig. 15. Because of the slow rising of the $z_{3}=\ln \left(\lambda_{t, b} / \lambda_{\max }\right) / \Delta \ln \epsilon$ distribution function in higher dimensions, the weight of the distribution of $z_{3}$ is shifted toward larger values, allowing for smaller values of $\lambda_{b}$.

As is evident from Fig. 15, however, the approximation $f(y) \propto y^{D / 2-1}$ is valid for only a very narrow range of $y$ near zero. Using the explicit form of $f(y)$ instead, we can extract another systematic $D$ dependence. The statistical average of $y_{i}=\ln \epsilon_{i} / \Delta \ln \epsilon$ plays the role of an AFS charge of the $i$ th generation, and $y_{i}-y_{j}$ determines the hierarchy between the $i$ th and $j$ th generations. For sequences of geometries with different numbers of extra dimensions$S^{1}, S^{2}$, and $S^{3}$, and $T^{1}=S^{1}, T^{2}$, and $T^{3}=S^{1} \times S^{1} \times S^{1}-$ an explicit calculation reveals

$$
\begin{aligned}
& \frac{\left\langle y_{1}-y_{2}\right\rangle}{\left\langle y_{2}-y_{3}\right\rangle}=1.50\left(S^{1}\right), \quad 1.44\left(S^{2}\right), \quad 1.38\left(S^{3}\right), \\
& \frac{\left\langle y_{1}-y_{2}\right\rangle}{\left\langle y_{2}-y_{3}\right\rangle}=1.50\left(T^{1}\right), \quad 1.20\left(T^{2}\right), \quad 1.14\left(T^{3}\right) .
\end{aligned}
$$

This ratio clearly decreases in Gaussian landscapes with a greater number of extra dimensions, meaning that the middle eigenvalue becomes (statistically) closer to the smallest eigenvalue on a logarithmic scale. This also means that in the AFS approximation the diagonalization angle (and hence the mixing angle) between the first and second generations becomes more significant compared with that between the second and third generations. This tendency is confirmed in Fig. 15, where the $\theta_{12}$ distribution becomes closer to the $\theta_{23}$ distribution for larger $D$. Given that we measure $\lambda_{d} / \lambda_{s}>\lambda_{s} / \lambda_{b}$ and $\theta_{12}>\theta_{23}$, this might be regarded as an indication that $D>1$. Note however that the compactness of extra dimensions affects the distributions of the smallest eigenvalues, and therefore the distributions of observables based on the AFS approximation cannot reliably be used to infer the number of extra dimensions.

Let us now summarize the conclusions of this section. The basic features of flavor in the standard model follow from Gaussian landscapes on any geometry of extra dimensions. Specifically, the distribution of Yukawa matrix elements is generally broad and thus there is always hierarchy among Yukawa mass eigenvalues. Furthermore, the up-type and down-type Yukawa matrices are correlated due to the common overlaps of left-handed quark doublets and the Higgs, so that generation structure is always realized. Even in the details of the distribution functions of flavor observables, there is not much geometry dependence. We understand this in terms of the AFS approximation, where all of the geometry dependence is encoded in the volume distribution function $f(y)$. This function is integrated and convoluted many times to obtain the distributions of observables, thus smearing out the original geometry dependence. This means that we cannot learn very much about the geometry of extra dimensions from the observed masses and mixing angles. On the other hand, it appears that we can understand the qualitative pattern of masses and mixing angles without knowing much about the details of the underlying geometry. The number of extra dimensions, however, leaves its footprint on some distribution functions near $y \sim 0$, because the boundary at $y=0$ remains a boundary in some convolutions. We also find that the mass eigenvalues of the second generation become closer (statistically) to those of the first generation as we increase the number of extra dimensions in Gaussian landscapes. 
For various geometries, the volume distribution functions $f(y)$ are usually moderately varying functions of $y$ over the range $y \in[0,1]$. Since the distribution functions of the AFS suppression factors $y_{i} \propto \ln \epsilon_{i}$, the mass eigenvalues $z_{i} \propto \ln \lambda_{i}$, and the mixing angles $t_{i j} \propto \ln \sin \theta_{i j}$ are all derived from $f(y)$, these distribution functions are moderately varying functions of the logarithmic variables $y_{i}, z_{i}$, and $t_{i j}$. The widths of these statistical distributions are quite broad; to achieve otherwise would require an exponentially steep $f(y)$, which does not happen in Gaussian landscapes. Therefore, the Gaussian landscapes in this article are different from those in [16], where all of the dimensionless coupling constants of the standard model have narrow-width Gaussian distributions. This feature of our toy landscapes is traced back to our assumption that the localized wave functions become exponentially small as one moves away from the centers of localization.

\section{INCLUDING THE LEPTON SECTOR}

Yukawa couplings generated from simple Gaussian landscapes are in good qualitative agreement with the mass hierarchies and small mixing angles in the quark sector. On the other hand, the lepton sector is characterized by large mixing angles and very small neutrino masses. We now turn our attention to how these qualitatively distinct features might be explained within a single landscape model. With the challenges of describing the lepton sector comes the opportunity to predict the probability distributions of three yet-to-be-measured observables in the lepton sector: the mixing angle $\theta_{13}$, the $C P$ phase in neutrino oscillations, and the mass parameter $m_{\beta \beta}$ of neutrinoless double beta decay.

In order to accommodate large mixing angles in the lepton sector, in Sec. VIA the Gaussian landscape is extended to include different widths for wave functions of particles in different representations. We will see in Sec. VID that the mixing angles can be large indeed, and we also learn that complex ( $C P$-violating) phases play a crucial role in determining the distribution of mixing angles (and vice versa in Sec. VIE). Before studying mixing angles we study the charged-lepton mass spectrum in Sec. VI B. Small neutrino masses are assumed to be generated via the seesaw mechanism, and in Sec. VIC we describe how to generate the statistics of the right-handed neutrino Majorana mass terms in Gaussian landscapes. Finally, in Sec. VIE we impose some cuts on the statistics generated by a particular version of the Gaussian landscape. This is done to obtain a general feeling for how the probability distributions of yet-to-be-measured observables can be affected when distributions conditional on measurements already performed in this universe are considered.

Note that our goal in this section is to find a single theoretical framework that can describe the various flavor structures that are observed; i.e. we aim to identify what subset of landscapes share key qualitative features with the observed flavor structure. Ultimately, the success of the landscape picture will depend on both the existence of phenomenologically viable subsets to the landscape and that these subsets are not too atypical of what is expected from the full landscape, after cosmological and environmental selection effects are accounted.

\section{A. Landscapes with delocalized $\overline{\mathbf{5}}=(\overline{\boldsymbol{d}}, \boldsymbol{l})$}

In the traditional AFS approach, the large mixing angles of neutrino oscillation and a mild charged-lepton mass hierarchy result if the three lepton doublets are not strongly distinguished by the flavor symmetry. For example, an AFS may be broken by a single parameter, with the three $q \subset \mathbf{1 0}=(q, \bar{u}, \bar{e})$ 's strongly distinguished by the symmetry charges while the three $l \subset \overline{\mathbf{5}}=(\bar{d}, l)$ 's are not. Since Gaussian landscapes can mimic an AFS structure in the Yukawa couplings, the idea of different strengths of flavor symmetry breaking for $\overline{\mathbf{5}}$ and $\mathbf{1 0}$ can be translated into the framework of Gaussian landscapes.

As is seen in Secs. III and V, the ratio of effective AFS charges for the three fermions in a given representation is determined (statistically) by the Gaussian landscape; we have no freedom to choose these by hand. On the other hand, the overall hierarchy depends on the parameter $\Delta \ln \epsilon \propto-(L / d)^{2}$. So far, for simplicity the parameter $d$ has been chosen to be the same for all of the standard model wave functions; however this parameter can be different for fields in different representations. If the $\mathbf{1 0}$ and $\overline{\mathbf{5}}$ fields have different width parameters $d_{\mathbf{1 0}}$ and $d_{\overline{\mathbf{5}}}$, then the AFS suppression factors associated with these fields are also different. In such a landscape the single parameter $d$ is replaced by three: $d_{H}, d_{\mathbf{1 0}}$, and $d_{\overline{\mathbf{5}}}$.

If the wave functions of the fermions in the $\overline{5}$ representation are not particularly localized, the overlaps between the wave functions of the $\overline{\mathbf{5}}$ fields and the Higgs boson do not vary hierarchically with the peak locations of the $\overline{\mathbf{5}}$ Gaussian wave functions. Therefore the Yukawa couplings associated with the three $\overline{\mathbf{5}}$ fields are not hierarchically separated when $d_{\overline{5}}$ is not much less than $L$. This is along the line of the idea in [17]. On the other hand, such a choice of $d_{5}$ maintains the hierarchical structure of the masses and mixing angles in the quark sector, when $d_{\mathbf{1 0}} / L$ and $d_{H} / L$ are chosen the same as before. The main exception is that the hierarchy of the down-type quark masses becomes a little smaller, as we see below, which is actually in good agreement with observation.

\section{B. Charged-lepton mass spectrum}

The masses of the charged leptons derive from the Yukawa interaction

$$
\mathcal{L}=\lambda_{a i}^{e} \bar{e}_{a} l_{i} h^{*},
$$

where $\lambda_{a i}^{e}$ is generated in analogy to (22), except with a large width $d_{\overline{5}}$ to the wave function of $l_{i}$. In the limit $d_{\overline{5}} \gg$ 

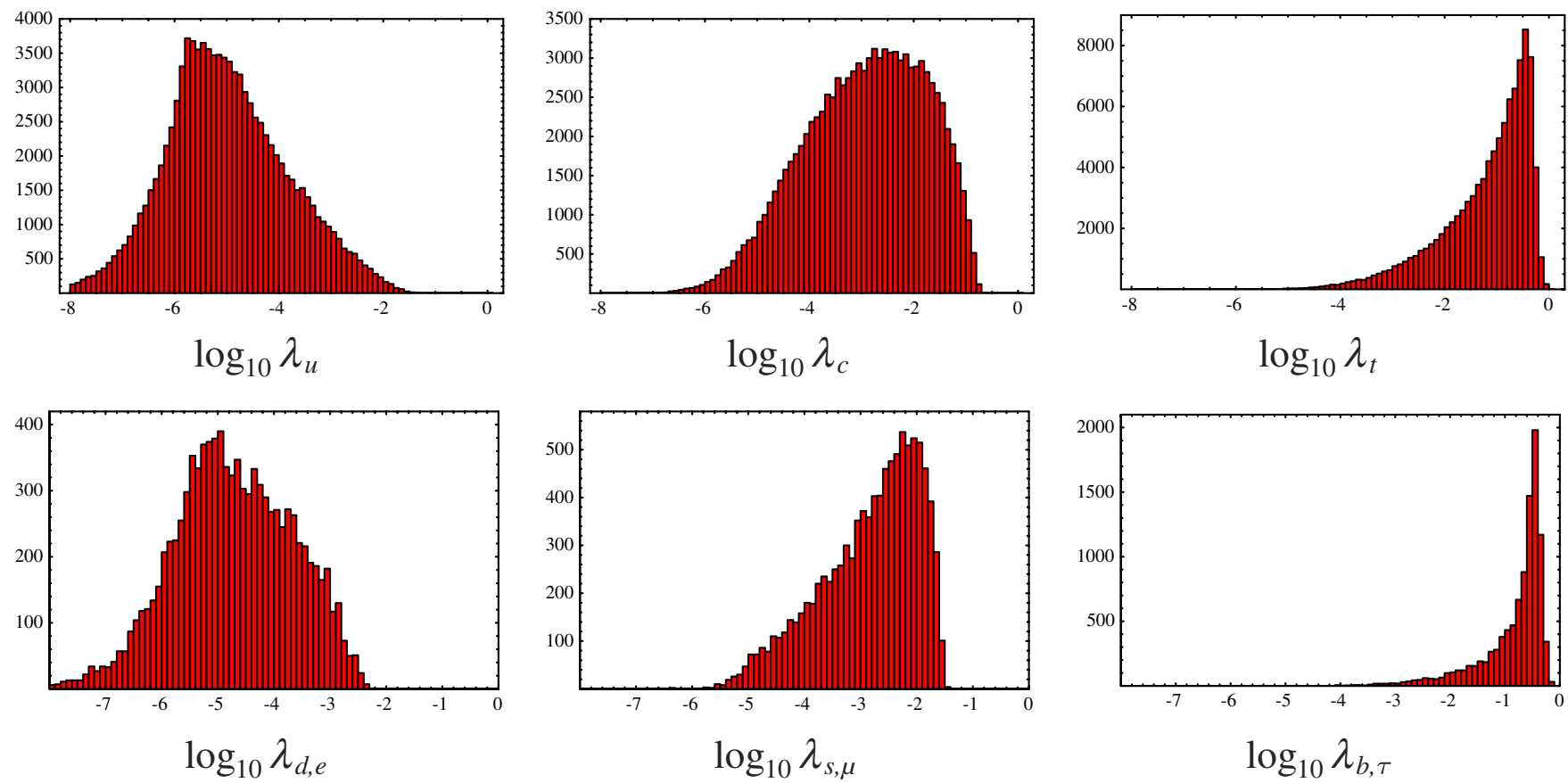

FIG. 16 (color online). Distributions for Yukawa eigenvalues from a Gaussian landscape on $S^{1}: g_{\text {eff }}=0.2$ for all overlap integrals and the Gaussian widths are $d / L=0.08$ for all fields except for $\bar{d}$ and $l$, which have $d_{\overline{5}} / L=0.3$.

$d_{\mathbf{1 0}}, d_{H}$ but still $d_{\overline{\mathbf{5}}} \approx L, \lambda_{a i}^{e}$ obtains an AFS structure

$$
\lambda_{a i}^{e} \sim \varphi_{i}^{l}\left(y=\frac{d_{H}^{2}}{d_{\mathbf{1 0}}^{2}+d_{H}^{2}} y_{a}^{\bar{e}} ; y_{i}^{l}\right) e^{-\left(\left(y_{a}^{\bar{e}}\right)^{2} / 2\left(d_{\mathbf{1 0}}^{2}+d_{H}^{2}\right)\right)}
$$

where $y_{a}^{\bar{e}}$ is the center coordinate of the $\bar{e}_{a}$ wave function relative that of the Higgs, and $\varphi_{i}^{l}\left(y ; y_{i}^{l}\right)$ is the broad-width wave function of $l_{i}$, centered at $y_{i}^{l}$. Scanning $y_{i}^{l}$ and $y_{a}^{\bar{e}}$, the first factor becomes a random coefficient of order unity for all elements of the $3 \times 3$ Yukawa matrix, while the second factor determines the flavor suppression. As the three eigenvalues are roughly the same as the three flavor suppression factors, the distributions of the three eigenvalues, $\lambda_{1,2,3}^{e}$, should be roughly the same as those of the three AFS suppression factors.

Therefore, in the Gaussian landscape on $S^{1}$ the shape of the distribution functions of the charged-lepton Yukawa eigenvalues should be like those in the left panel of Fig. 6. We will discuss the overall range of the hierarchy shortly. If we use a common width parameter for $\mathbf{1 0}=(q, \bar{u}, \bar{e})$ and a different common width for $\overline{\mathbf{5}}=(\bar{d}, l),{ }^{15}$ then the distributions of eigenvalues for the down quark sector should be the same as those of the charged-lepton sector. On the other hand, the up-sector Yukawa couplings involve two fields in the 10 representation, so the distribution of eigenvalues should be closer to those in the central panel of Fig. 6 (see

\footnotetext{
${ }^{15}$ It is an interesting theoretical question whether higherdimensional field theories can give rise to independent scanning of the centers of each of the $q, \bar{u}, \bar{d}, l, \bar{e}$ wave functions while preserving the $\mathrm{SU}(5)_{\mathrm{GUT}}$ symmetric widths.
}

Sec. III regarding the limitations of applying the AFS approximation to small eigenvalues). The results of a numerical simulation, displayed in Fig. 16, confirm that the AFS approximation captures the width-parameter dependence of the distribution functions. Although we do not present numerical results for Gaussian landscapes other than on $S^{1}$, the approximate analytic distribution functions for the AFS suppression factors on $T^{2}, S^{2}$, and $S^{3}$ are presented in Fig. 15. In principle one can generalize the analytic discussion to many geometries.

In the limit of broad $\overline{\mathbf{5}}$ wave functions, $d_{\overline{\mathbf{5}}} \gg d_{\mathbf{1 0}}, d_{H}$ (but $d_{\overline{5}} \approx L$ so that values of $\varphi_{i}^{l}$ remain random), the logarithmic range covered by the charged-lepton Yukawa eigenvalues is

$$
\Delta \ln \epsilon=-\frac{L^{2}}{8\left(d_{10}^{2}+d_{H}^{2}\right)} .
$$

For simplicity we choose $d_{\mathbf{1 0}}$ and $d_{H}$ to be the same, giving $\Delta \ln \epsilon=-(L / d)^{2} / 16$. This is $3 / 4$ of the result $\Delta \ln \epsilon=$ $-(L / d)^{2} / 12$ when all of the three widths are the same. In the numerical results of Fig. 16, the overall hierarchy in the up sector is slightly larger than that of the down/chargedlepton sectors, consistent with these analytic expectations.

In Gaussian landscapes with $d_{\overline{\mathbf{5}}} \gg d_{\mathbf{1 0}}, d_{H}, \lambda_{b, \tau}$ tend to be predicted larger than $\lambda_{t}$. This is of course a problem if there is no factor such as $\tan \beta$ in the two-Higgs doublet model. This problem can be solved in two different ways. One is that there may be environmental selection in favor of a large top-Yukawa coupling, which makes the observed top-Yukawa coupling at the far upper end of its prior 
distribution, while the bottom and tau Yukawa couplings are not. This scenario does not work very well in the Gaussian landscape on $S^{1}$, but it may work in Gaussian landscapes in higher dimensions, since the distributions of the largest eigenvalues receive a high-end tail in $D>1$ extra dimensions (see Sec. V). The other solution is that the coefficient $g_{\text {eff }}$ of overlap integration is smaller for the down-type/charged-lepton sectors than that for the uptype sector. Since the overall hierarchy for the up-type sector is larger than in the down and charged-lepton sectors, and because the distributions for the smallest eigenvalues are very broad, this can be done while still maintaining the order of magnitude agreement between $\lambda_{d, e}$ and $\lambda_{u}$.

It is interesting to note a difference between Gaussian landscapes with large $d_{\overline{5}}$ and the AFS models that accomplish neutrino anarchy with very weak AFS for the lepton doublets. In these AFS models, the logarithmic range of the hierarchy in the down-type/charged-lepton sectors is half of that of the up-type sector. On the other hand, this ratio is typically about 3/4 in the Gaussian landscape on $S^{1}$ (when $d_{\mathbf{1 0}}=d_{H}$ ). Of course the precise number $3 / 4$ may be different for different numbers of dimensions, geometry, and choice of $d_{10} / d_{H}$. The difference between AFS and Gaussian landscapes stems from the fact that in the latter the AFS suppression factors associated with $\mathbf{1 0}$ are different for the up-type and down-type/charged-lepton Yukawa matrices,

$$
\begin{aligned}
\boldsymbol{\epsilon}^{q, \bar{u}(\mathrm{u}-\text { sector })} & \sim e^{-\left(d_{\mathbf{1 0}}^{2}+d_{H}^{2} / d_{\mathbf{1 0}}^{2}+2 d_{H}^{2}\right)\left(y_{a}^{2} / 2 d_{\mathbf{1 0}}^{2}\right)}, \\
\boldsymbol{\epsilon}^{q, \bar{e}(\mathrm{~d} / \mathrm{e}-\text { sector })} & \sim e^{-\left(y_{a}^{2} / 2\left(d_{\mathbf{1 0}}^{2}+d_{H}^{2}\right)\right)} .
\end{aligned}
$$

In other words, the flavor suppression factors are not determined by symmetry charges intrinsically assigned to fields in the $\mathbf{1 0}$ representation, but depend on the nature of the other fields. In our universe, the down-type/chargedlepton sector hierarchy is not as small as half of that of the up sector, and Gaussian landscapes have enough flexibility to accommodate this situation.

\section{Neutrino mass hierarchy}

Very small neutrino masses are obtained via the seesaw mechanism. Specifically, we assume left-handed neutrino masses derived from the effective dimension-five operators,

$$
\mathcal{L}_{\text {eff }}=\frac{C_{i j}}{M} l_{i} l_{j} h h,
$$

which are generated after integrating out heavy righthanded neutrinos, with interactions

$$
\begin{aligned}
\mathcal{L} & =M c_{\alpha \beta} \bar{\nu}_{R \alpha} \bar{\nu}_{R \beta}+\lambda_{\alpha i}^{\nu} \bar{\nu}_{R \alpha} l_{i} h ; \\
C_{i j} & =\left(\lambda^{\nu T} c^{-1} \lambda^{\nu}\right)_{i j} .
\end{aligned}
$$

In a Gaussian landscape the neutrino Yukawa couplings $\lambda_{\alpha i}^{\nu}$ are generated by scanning the center coordinates of
Gaussian wave functions, analogous to the generation of $\lambda_{i j}^{u, d}$. Yet the low-energy observables in the neutrino sector also depend on $M c_{\alpha \beta}$, so we need to introduce some additional assumptions regarding how the Majorana mass terms of the right-handed neutrinos are generated. The traditional approach has been to assume an AFS pattern in the mass matrix $M c_{\alpha \beta}$. However, our Gaussian landscapes are not based on the breaking of any flavor symmetry, but on the overlap integration of localized wave functions. Therefore we incorporate this principle into the generation of right-handed Majorana mass terms.

Right-handed neutrinos are singlets under the standard model gauge group, but they are charged in more unified gauge groups. ${ }^{16}$ For example, unified gauge groups such as $\mathrm{SU}(4)_{C} \times \mathrm{SU}(2)_{L} \times \mathrm{SU}(2)_{R}, \mathrm{SO}(10)$, and $E_{6} \rightarrow E_{8}$ contain a $B-L$ symmetry, and the right-handed neutrinos carry nonvanishing charges under this symmetry. In order for charged particles to have Majorana mass terms, some scalar fields on $3+1$ dimensions have to be inserted to form gauge-invariant operators, and the expectation values of these scalar fields convert the operators into Majorana mass terms:

$$
\mathcal{L}=\left\langle\phi_{\mathrm{SB}}\right\rangle \bar{\nu}_{R} \bar{\nu}_{R}, \quad \text { or } \quad \mathcal{L}=\left\langle\phi_{\mathrm{SB}}^{\prime} \phi_{\mathrm{SB}}^{\prime}\right\rangle \bar{\nu}_{R} \bar{\nu}_{R} .
$$

The scalar fields $\phi_{\mathrm{SB}}$ or $\phi_{\mathrm{SB}}^{\prime}$ are singlets of the standard model gauge groups, but carry charges under whatever symmetry the right-handed neutrinos are charged. In other words, they are moduli fields describing the symmetry breaking of the more unified symmetry.

Since the Majorana mass terms (74) involve vacuum expectation values of scalar fields, the coefficients of the mass terms will involve overlap integrations:

$$
M c_{\alpha \beta} \propto \int d y \varphi^{\mathrm{SB}} \varphi_{\alpha}^{\bar{\nu}_{R}} \varphi_{\beta}^{\bar{\nu}_{R}},
$$

where $\varphi_{\alpha}^{\bar{\nu}_{R}}$ is the zero-mode wave function of the $\alpha$ copy of the right-handed neutrino and $\varphi^{\mathrm{SB}}$ represents all of the other effects of localization in the extra dimension(s), including the wave functions of the symmetry-breaking moduli fields $\phi_{\mathrm{SB}}$ and/or $\phi_{\mathrm{SB}}^{\prime}$. These fields have nothing to do with the Higgs boson, and hence there is no reason to believe that $\varphi^{\mathrm{SB}}$ is localized at the same place in the extra dimensions as the Higgs boson wave function. Thus while the AFS structure of all of the Yukawa couplings $\lambda^{u, d, e, \nu}$ is due to an overlap between fermion wave functions and that of the Higgs boson, any flavor structure in the Majorana mass term follows from an overlap involving $\varphi^{\mathrm{SB}}$. In particular, unless there is a strong correlation between the wave functions of $\varphi^{\mathrm{SB}}$ and the Higgs boson, the flavor structure of the mass matrix $M c_{\alpha \beta}$ is statistically independent of that of the Yukawa matrices.

\footnotetext{
${ }^{16}$ The term "unified gauge group" is here used in a loose sense. For instance, we do not assume that the standard model gauge group is unified as an effective field theory in $3+1$ dimensions.
} 
We study the consequences of this new flavor structure in Secs. VIC 1 and VIC 2. However, before we begin some comments are in order. Our first remark concerns the overall mass scale $M$ of the right-handed neutrinos. The overlap integration in (75) sets the flavor structure of $M c_{\alpha \beta}$, but does not say anything about the overall scale of the symmetry breaking. Thus an extra assumption has to be introduced in order to set the distribution of the overall scale $M$. Even if we know this distribution from theoretical considerations, it may be modulated by environmental selection related to leptogenesis. Therefore we set aside this issue and instead focus only on the hierarchy among neutrino masses and mixing angles in the lepton sector, which can be determined independent of the overall scale of the neutrino masses. This is equivalent to studying a fixed $M$ cross section of the full landscape. Our second comment concerns the form of the wave functions of the righthanded neutrinos and the symmetry-breaking source $\varphi^{\mathrm{SB}}$. To date we have very little knowledge about these fields; yet in a Gaussian landscape their most crucial aspect will be whether in the extra dimensions their wave functions are localized or not. Therefore we set aside the theoretical origin of these fields and simply represent them with Gaussian wave functions (20), while considering the width parameters $d_{N}$ and $d_{\mathrm{SB}}$ as unknown. By choosing $d_{N}$ and $d_{\mathrm{SB}}$ to be large or small, we can simulate various possibilities. For example, Majorana mass terms generated by world-sheet instantons wrapped on topological cycles may be mimicked by a (possibly multicentered) Gaussian wave function with a small $d_{\mathrm{SB}}$.

It turns out that the mass matrices of the neutrino sector are quite different depending on whether the right-handed neutrinos have localized wave functions or not. Therefore Secs. VIC 1 and VIC 2 are separately devoted to these two possibilities. In either case, to generate a statistical ensemble of $\lambda_{\alpha i}^{\nu}$ and $c_{\alpha \beta}$ the center coordinates of wave functions are scanned randomly and independently for $\varphi^{\mathrm{SB}}$, the right-handed neutrinos, lepton doublets, and the Higgs boson.

\section{Narrow right-handed neutrino wave functions}

If right-handed neutrinos have narrow wave functions, then the neutrino Yukawa matrix has a structure similar to that of charged leptons. That is, we have

$\lambda_{\alpha i}^{\nu} \sim \varphi_{i}^{l}\left(y=\frac{d_{H}^{2}}{d_{N}^{2}+d_{H}^{2}} y_{\alpha}^{\bar{\nu}_{R}} ; y_{i}^{l}\right) e^{-\left(\left(y_{\alpha}^{\bar{\nu}_{R}}\right)^{2} / 2\left(d_{N}^{2}+d_{H}^{2}\right)\right)} \equiv g_{\alpha i} \epsilon_{\alpha}^{D}$.

This is (69) with $d_{\mathbf{1 0}}$ replaced by $d_{N}$; and again the $g_{\alpha i}$ are effectively random coefficients of order unity. The range of the hierarchy is $\Delta \ln \epsilon^{D}=-L^{2} /\left[8\left(d_{N}^{2}+d_{H}^{2}\right)\right]$.

Meanwhile, the Majorana mass matrix $c_{\alpha \beta}$ of righthanded neutrinos is almost diagonal, because the offdiagonal entries are suppressed due to the (statistically) small overlap of wave functions between different righthanded neutrinos. If the symmetry-breaking source $\varphi^{\mathrm{SB}}$ is also very localized, then the Majorana mass matrix has the approximate structure

$$
c_{\alpha \beta} \sim \delta_{\alpha \beta} e^{-\left(\left(y_{\alpha}^{\bar{\nu}_{R}}-y^{S B}\right)^{2} / 2 d_{S B}^{2}+d_{N}^{2}\right)} \equiv \delta_{\alpha \beta} \epsilon_{\alpha}^{M},
$$

where $d_{\mathrm{SB}}$ is the width of Gaussian wave function of $\varphi^{\mathrm{SB}}$ and $y^{\mathrm{SB}}$ is the center coordinate of this wave function. The positions $y_{\alpha}^{\bar{\nu}_{R}}$ and $y^{\mathrm{SB}}$ are scanned randomly and the largest, middle, and smallest $\epsilon_{\alpha}$ become the three eigenvalues of the right-handed neutrino mass matrix. In particular, their distribution functions should be like those of the AFS suppression factors $\epsilon_{1,2,3}$ in Fig. 6 or 15. For Gaussian landscapes on $S^{1}$ we have $\Delta \ln \epsilon^{M}=-L^{2} /\left[4\left(2 d_{S B}^{2}+\right.\right.$ $\left.d_{N}^{2}\right)$ ]. Results of a numerical simulation on the $S^{1}$ landscape are shown in the first row of Fig. 17, confirming the theoretical expectations so far. If the symmetry-breaking source $\varphi^{\mathrm{SB}}$ does not have a localized wave function, then we can take $d_{\mathrm{SB}}$ to be very large. Then $\Delta \ln \epsilon^{M} \approx 0$, meaning that there is not much hierarchy among the mass eigenvalues of right-handed neutrinos.

From (73), (76), and (77) the mass matrix of low-energy neutrinos is approximately

$$
C_{i j} \sim \sum_{\alpha} g_{\alpha i} g_{\alpha j} \frac{\left(\epsilon_{\alpha}^{D}\right)^{2}}{\epsilon_{\alpha}^{M}} .
$$

Since we introduce no correlation between the Higgs and $\varphi^{\mathrm{SB}}$, we do not expect net statistical cancellations in the ratio $\left(\epsilon_{\alpha}^{D}\right)^{2} / \epsilon_{\alpha}^{M}$. This contrasts sharply with flavor theories that assume an AFS structure for the Majorana mass matrix of right-handed neutrinos. Let the distributions of $\ln \epsilon^{D}$ and $\ln \epsilon^{M}$ be denoted by

$$
d P\left(y^{D}\right)=f_{D}\left(y^{D}\right) d y^{D}, \quad d P\left(y^{M}\right)=f_{M}\left(y^{M}\right) d y^{M},
$$

where we introduce the new variables $y^{D, M} \equiv$ $\ln \epsilon^{D, M} / \Delta \ln \epsilon^{D} \quad\left(y^{D}\right.$ is normalized to run from zero to one; note that the variable $y^{M}$ does not necessarily run from zero to one). Because of the form of $\epsilon^{D}$ and $\epsilon^{M}$ in (76) and (77), $f_{D}(y)$ and $f_{M}(y)$ are given by the volume distribution function $f(y)$ of (60), after renormalizing the distribution function and rescaling the argument, if necessary. To approximate the distributions of eigenvalues of $\ln C_{i j}$, we first introduce the variable $z^{\mathrm{ss}} \equiv \ln \left[\left(\epsilon^{D}\right)^{2} / \epsilon^{M}\right] / \Delta \ln \epsilon^{D}$. The distribution of $z_{\mathrm{ss}}$ follows from appropriately convoluting the distributions of $\ln \epsilon^{D}$ and $\ln \epsilon^{M}$,

$$
\frac{d P\left(z^{\mathrm{ss}}\right)}{d z^{\mathrm{ss}}} \equiv f_{\mathrm{ss}}\left(z^{\mathrm{ss}}\right)=\int \frac{1}{2} f_{D}(z / 2) f_{M}\left(z-z^{\mathrm{ss}}\right) d z .
$$

Note that the variable $z^{\text {ss }}$ runs from $-2\left(d_{H}^{2}+d_{N}^{2}\right) /\left(2 d_{S B}^{2}+\right.$ $d_{N}^{2}$ ) to 2 on $S^{1}$. The distribution function $f_{\text {ss }}$ can be calculated explicitly once the underlying volume distribution function $f(y)$ is known; the results for the Gaussian landscapes on $S^{1}$ and $S^{2}$ are shown in Fig. 18. Using the volume distribution function $f_{\mathrm{ss}}\left(z_{\mathrm{ss}}\right)$, we obtain the distributions of 

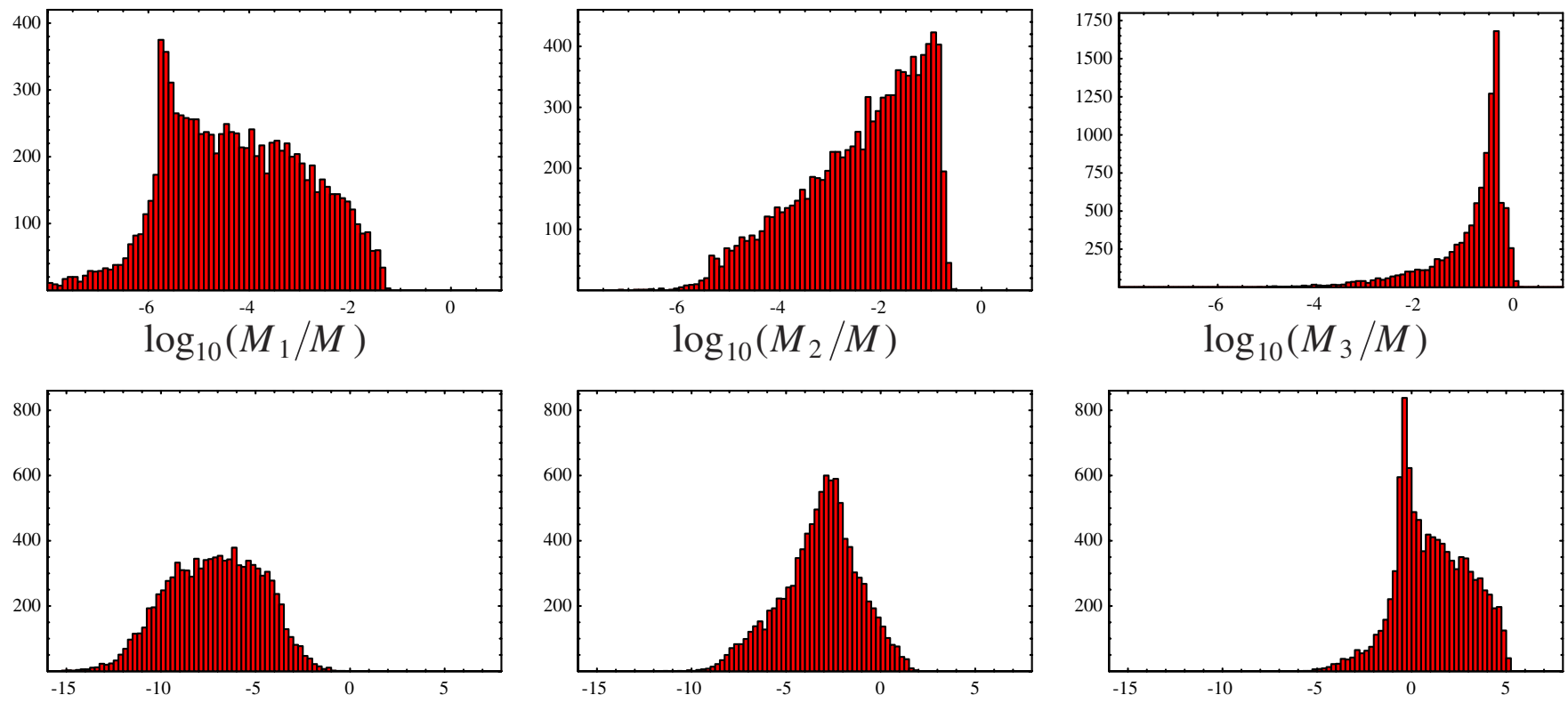

$\log _{10}\left(m_{1} / m_{\nu}\right)$
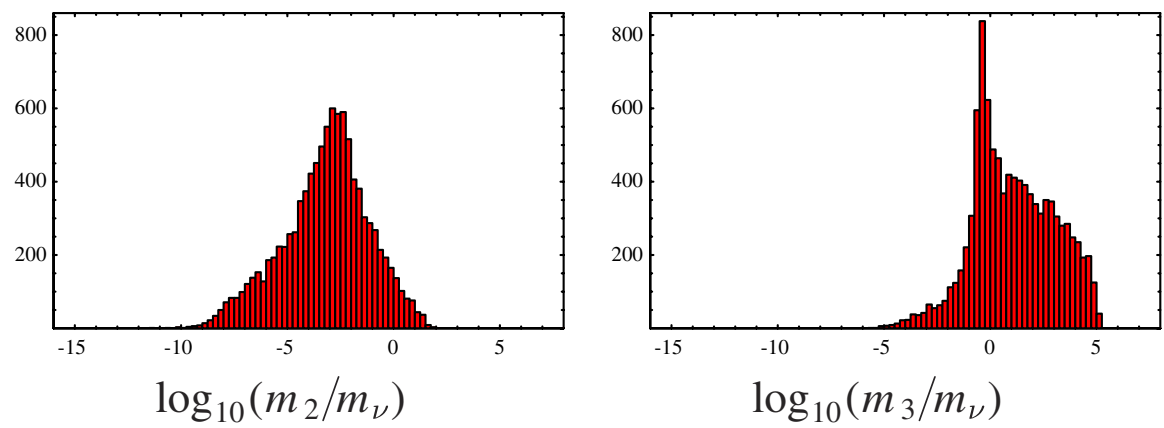

FIG. 17 (color online). Distributions of the eigenvalues of the right-handed neutrino mass matrix and the left-handed seesaw Majorana mass matrix, normalized by $M$ and $m_{\nu} \equiv\langle h\rangle^{2} / M$, respectively. The Gaussian landscape on $S^{1}$ is used for this simulation, with $d / L=0.08$ for the wave functions of $\bar{\nu}_{R \alpha}, \varphi^{\mathrm{SB}}$, and the Higgs boson while $d / L=0.3$ for the lepton doublets.
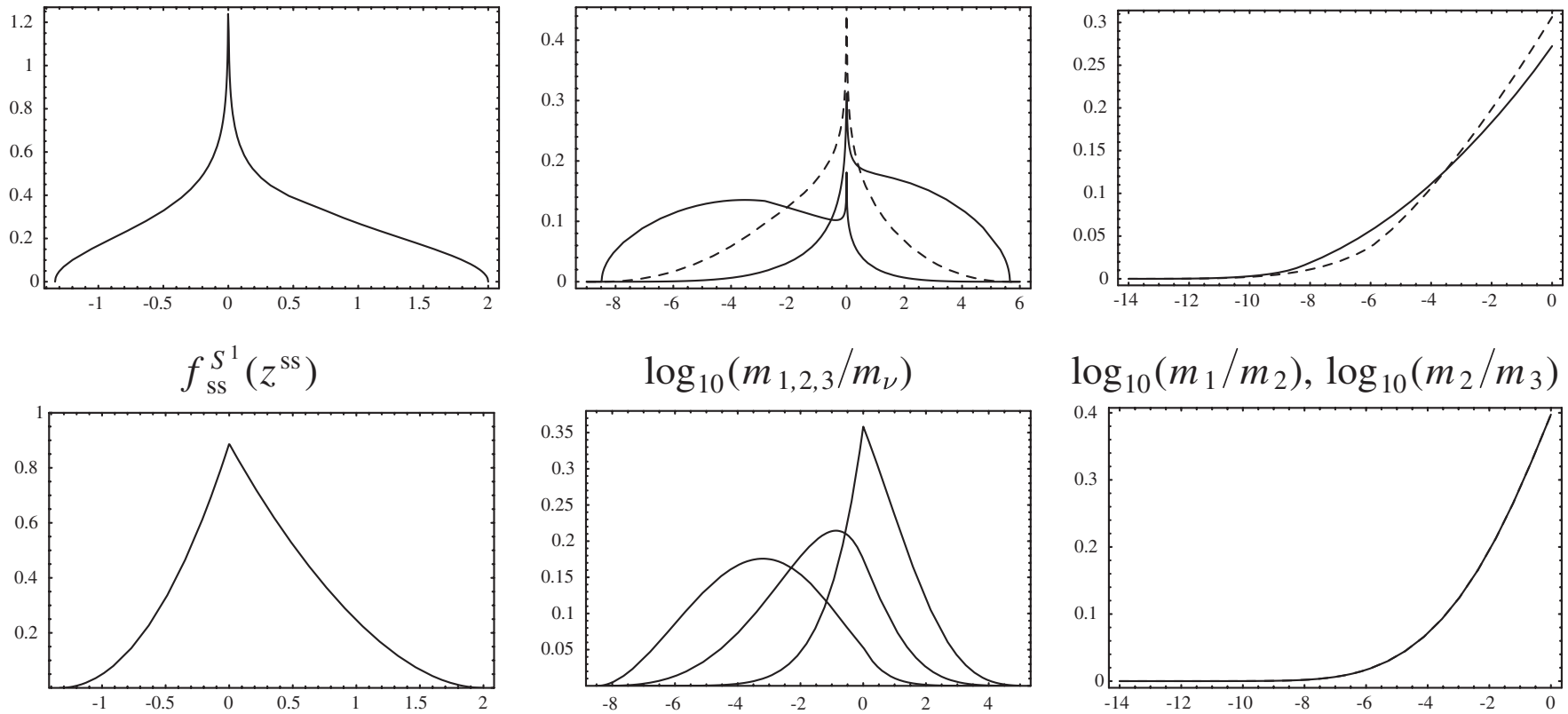

$\log _{10}\left(m_{1} / m_{2}\right), \log _{10}\left(m_{2} / m_{3}\right)$

$$
f_{\mathrm{ss}}^{S^{2}}\left(z^{\mathrm{ss}}\right)
$$

$\log _{10}\left(m_{1,2,3} / m_{\nu}\right)$

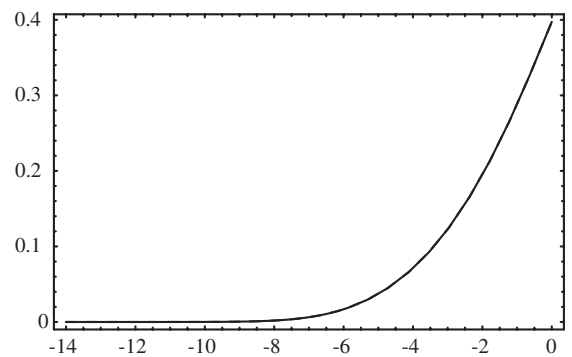

$\log _{10}\left(m_{1} / m_{2}\right), \log _{10}\left(m_{2} / m_{3}\right)$

FIG. 18. Distributions of density functions $f_{\mathrm{ss}}\left(z^{\mathrm{ss}}\right)$ (left column), low-energy left-handed neutrino eigenvalues (middle column), and the mass ratios $\left(m_{1} / m_{2}\right)$ and $\left(m_{2} / m_{3}\right)$ (right column). The top row is for $S^{1}$ while the bottom row is for $S^{2}$ [to reduce computation time we have approximated $f_{S^{2}}$ with a polynomial that is always within a part in a thousand of the corresponding expression in (61)]. In the top row the distributions for the middle eigenvalue $m_{2} / m_{\nu}$ and the ratio $m_{2} / m_{3}$ are displayed with a dashed line. The two mass ratios are the same on $S^{2}$. For these curves $d_{H} / L=d_{N} / L=d_{\mathrm{SB}} / L=0.08$. 
the three eigenvalues of $C_{i j}$ by integrating the other two variables out of the combined distribution ${ }^{17}$

$$
\begin{aligned}
d P\left(z_{3}^{\mathrm{ss}}, z_{2}^{\mathrm{ss}}, z_{1}^{\mathrm{ss}}\right)= & 3 ! f_{\mathrm{ss}}\left(z_{3}^{\mathrm{ss}}\right) f_{\mathrm{ss}}\left(z_{2}^{\mathrm{ss}}\right) f_{\mathrm{ss}}\left(z_{1}^{\mathrm{ss}}\right) \Theta\left(z_{1}^{\mathrm{ss}}-z_{2}^{\mathrm{ss}}\right) \\
& \times \Theta\left(z_{2}^{\mathrm{ss}}-z_{3}^{\mathrm{ss}}\right) d z_{3}^{\mathrm{ss}} d z_{2}^{\mathrm{ss}} d z_{1}^{\mathrm{ss}},
\end{aligned}
$$

where $\quad z_{1,2,3}^{\mathrm{ss}}=-8\left[\left(d_{H}^{2}+d_{N}^{2}\right) / L^{2}\right] \ln \left(m_{1,2,3} / m_{\nu}\right)$, with $m_{\nu} \equiv\langle h\rangle^{2} / M$. Distribution functions of other observables such as a ratio of low-energy neutrino masses are also calculated from this combined probability distribution. Some examples are displayed in Fig. 18. Note that the low-energy neutrino masses tend to have a very large hierarchy, because the distribution function $f_{\mathrm{ss}}$ covers the enormous logarithmic range

$$
\begin{aligned}
\Delta \ln m_{\nu} & =2 \Delta \ln \epsilon^{D}+\Delta \ln \epsilon^{M} \\
& =-\frac{1}{4}\left(\frac{L^{2}}{d_{N}^{2}+d_{H}^{2}}+\frac{L^{2}}{2 d_{S B}^{2}+d_{N}^{2}}\right) .
\end{aligned}
$$

Specifically, the hierarchies of neutrino Yukawa matrix and the Majorana mass matrix add rather than cancel, c.f. $[8,12]$. Even when $d_{S B}$ is very large, low-energy neutrino masses have a hierarchy twice as large as that of eigenvalues of the neutrino Yukawa matrix.

The low-energy neutrino eigenvalues of Fig. 17 show a remarkable similarity ${ }^{18}$ in shape with the corresponding distributions in Fig. 18, confirming that the theoretical derivation of distribution functions is fairly reliable. Although results of a numerical simulation on the Gaussian landscape on $T^{2}$ are not presented here, we have confirmed that the distributions of $\ln \left(m_{i} / m_{\nu}\right)$ are similar to corresponding distributions in Fig. 18. This success arises because the overlap integration for $\lambda_{a i}^{e}$, $\lambda_{\alpha i}^{\nu}$, and $c_{\alpha \beta}$ involves only two very localized wave functions. Although we have seen around (37) that the analytic distribution functions based on the volume distribution function have a systematic error due to the compactness of the extra dimension(s), the error occurs only for overlap integrations involving three localized Gaussian wave functions. Therefore, these analytic results can be used to compute distribution functions of flavor observables in the lepton sector in other geometries for which numerical simulation is costly.

The underlying geometry is reflected in the volume distribution functions $f_{D}$ and $f_{M}$. However, these are inte-

\footnotetext{
${ }^{17}$ The combined probability distribution of neutrino mass eigenvalues is discussed in [9] for the case with $f_{D}$ and $f_{M}$ proportional to a $\delta$ function. Reference [12] deals with the neutrino anarchy scenario, where the diagonalization effects on the eigenvalue distribution are also taken into account.

${ }^{18}$ The peak of the distribution of $\log _{10} m_{2} / m_{\nu}$ in Fig. 17 is near -2 , as opposed to the expected location 0 , shown in Fig. 18. The peak of $m_{2} / m_{\nu}$ being lower than that of $m_{3} / m_{\nu}$ is not surprising. We have seen similar phenomena in Figs. 5, 11, 14, and 16. As mentioned earlier, these are likely due to the diagonalization effect.
}

grated once to obtain $f_{\text {ss }}$, which smears the effects of geometry. The original $f$ 's are integrated at least once in the distribution functions of $\ln \left(m_{i} / m_{\nu}\right)$, like those of the up-sector eigenvalues. Moreover, the distribution functions of observables such as $\ln \left(m_{1} / m_{2}\right)$ and $\ln \left(m_{2} / m_{3}\right)$ involve two integrations over the $f$ 's, such that very little geometry dependence is left in the distribution of the observable. ${ }^{19}$

\section{Broad right-hand neutrino wave functions}

If the zero-mode wave functions of right-handed neutrinos are not localized, i.e. the width-parameter $d_{N}$ is not much less than $L$, then in the neutrino Yukawa coupling only the Higgs boson has a localized wave function. Thus the overlap integral is evaluated around the peak of the Higgs wave function. Expanding the other wave functions,

$$
\varphi_{j}^{l}(y)=\varphi_{j}^{l}\left(y^{h}\right)+\varphi_{j}^{l \prime}\left(y^{h}\right)\left(y-y^{h}\right)+\frac{1}{2} \varphi_{j}^{l \prime \prime}\left(y^{h}\right)\left(y-y^{h}\right)^{2},
$$

$$
\begin{aligned}
\varphi_{\alpha}^{\bar{\nu}_{R}}(y)= & \varphi_{\alpha}^{\bar{\nu}_{R}}\left(y^{h}\right)+\varphi_{\alpha}^{\bar{\nu}_{R}}\left(y^{h}\right)\left(y-y^{h}\right) \\
& +\frac{1}{2} \varphi_{\alpha}^{\bar{\nu}_{R} \prime \prime}\left(y^{h}\right)\left(y-y^{h}\right)^{2},
\end{aligned}
$$

we see that the neutrino Yukawa matrix is of the form

$$
\lambda_{i \alpha}^{\nu} \propto \varphi_{i}^{l} \varphi_{\alpha}^{\bar{\nu}_{R}}+\frac{1}{2}\left[\varphi_{i}^{l \prime \prime} \varphi_{\alpha}^{\bar{\nu}_{R}}+\varphi_{i}^{l} \varphi_{\alpha}^{\bar{\nu}_{R} \prime \prime}+2 \varphi_{i}^{l \prime} \varphi_{\alpha}^{\bar{\nu}_{R}}\right] d_{H}^{2}+\ldots,
$$

where all of the wave functions are evaluated at $y^{h}$. Note that the first term is a rank one matrix while the additional terms are suppressed by $\left(d_{H} / \min \left\{d_{N}, d_{\overline{\mathbf{5}}}\right\}\right)^{2}$. Thus we obtain a neutrino Yukawa matrix that has the form of the "democratic" mass matrix ansatz, i.e. it is rank one at leading order [18]. Deviations from absolute democracy (i.e. from being rank one) result from the wave functions for $l_{i}$ and $\bar{\nu}_{R \alpha}$ not being absolutely flat. The first row of Fig. 19 shows the distributions of the three eigenvalues of the neutrino Yukawa matrix. Note that $\varphi, \varphi^{\prime}$, and $\varphi^{\prime \prime}$ have upper limits and there are no effects enhancing the middle eigenvalue; hence the sharp cutoff of the distribution of $\ln \lambda_{2}^{\nu}$. An interesting conclusion is that the neutrino Yukawa matrix has a certain amount of hierarchy, no matter how large or small the width-parameter $d_{N}$ of the right-handed neutrinos is. Small Yukawa couplings result from small overlap integrals for localized right-handed neutrinos, or from rank reduction for nonlocalized right-handed neutrinos.

\footnotetext{
${ }^{19}$ The cuspy peaks in the distributions of $f_{\text {ss }}$ and $\ln \left(m_{i} / m_{\nu}\right)$ on $S^{1}$ are only logarithmic; the $y^{-1 / 2}$ singularity of the original $f_{S^{1}}$ is integrated at least once in each of these. We chose a fine binning in Fig. 17 in order to accentuate the logarithmic singularity and emphasize the agreement between the simulation and analytic derivation. When a coarser binning is chosen, the logarithmic singularities are smeared out. Thus, there is not much practical difference in the distributions of $\ln \left(m_{i} / m_{\nu}\right)$ 's whether $S^{1}$ or $T^{2}$ or any other geometry is used.
} 

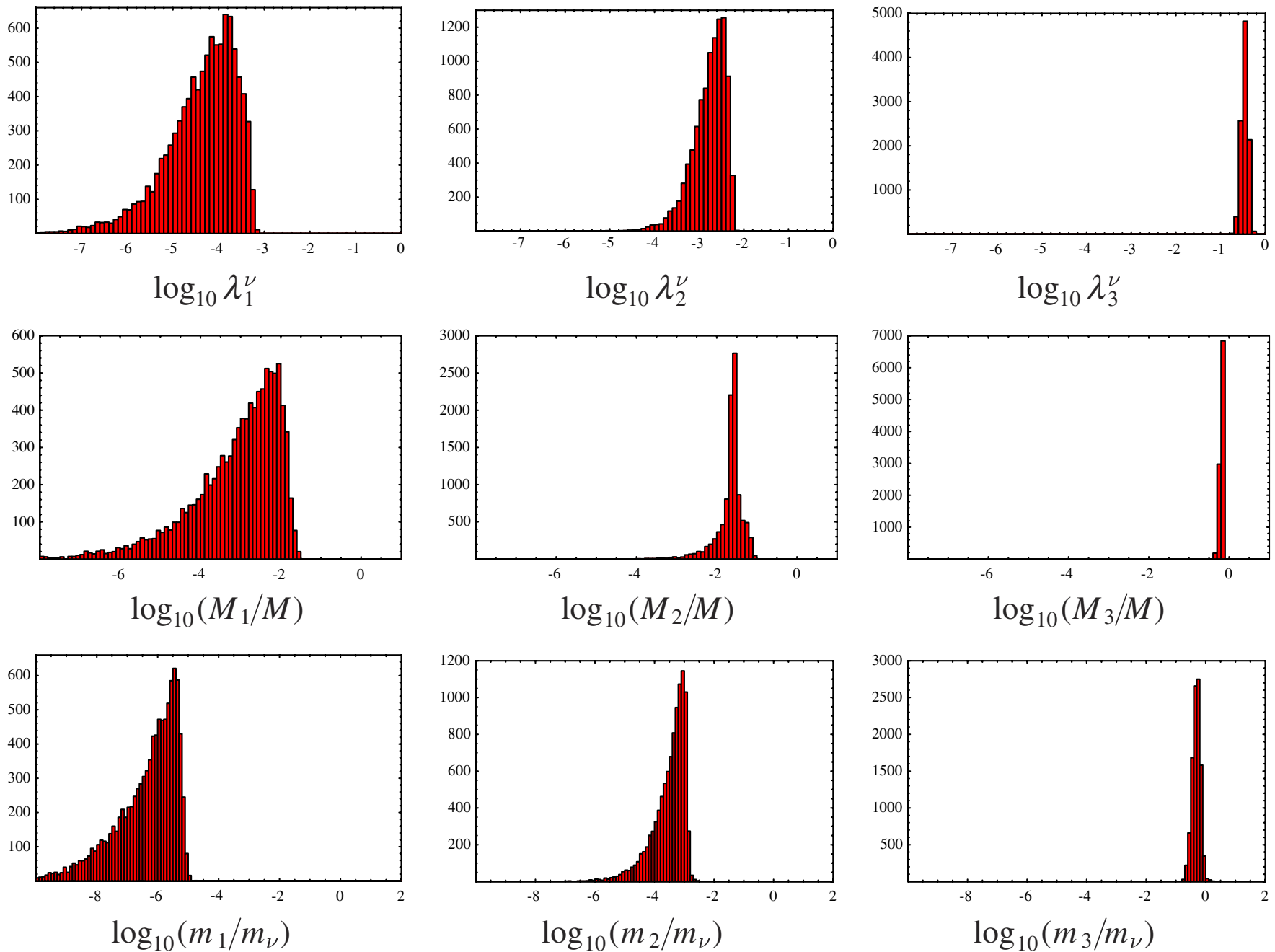

FIG. 19 (color online). Distributions of the eigenvalues of the left-handed neutrino Yukawa matrix, the right-handed Majorana mass matrix, and the low-energy left-handed Majorana mass matrix. The numerical simulation uses a Gaussian landscape on $S^{1}$, with $d_{H} / L=0.08$ and $d_{\mathrm{SB}} / L=d_{N} / L=d_{\overline{5}} / L=0.3$.

The Majorana mass matrix of right-handed neutrinos is not hierarchical when the source of symmetry breaking $\varphi^{\mathrm{SB}}$ is not localized. Thus in the second row of Fig. 19 the distribution of $M_{2} / M$ is roughly order unity, and the hierarchy $M_{2} / M_{3}$ is not more than what is expected from the diagonalization effect. ${ }^{20}$ On the other hand, the Majorana masses of right-handed neutrinos become hierarchical when $\varphi^{\mathrm{SB}}$ is localized. Then $c_{\alpha \beta}$ is rank one at leading order, and deviation from being rank one should come at the order of $\left(d_{\mathrm{SB}} / d_{N}\right)^{2}$.

The third row of Fig. 19 shows the distribution of mass eigenvalues of the low-energy seesaw neutrinos for $d_{N} / L=d_{S B} / L=0.3$. The distribution of $\ln \left(m_{2} / m_{\nu}\right)$ is sharply cutoff at its largest value; this cutoff is presumably traced back to the similarly sharp cutoff of the distribution of the second largest eigenvalue of the neutrino Yukawa

\footnotetext{
${ }^{20}$ We do not have a clear explanation why the distribution of $M_{1} / M$ has a long tail toward smaller values.
}

matrix $\lambda_{2}^{\nu}$. As opposed to when the right-handed neutrinos have localized wave functions, it is not as straightforward to develop an analytical understanding of the distribution of seesaw neutrino mass eigenvalues when the righthanded neutrinos have nonlocalized wave functions. Instead, we run numerical simulations with the Gaussian landscape on $S^{1}$, using a range of $d_{N} / L \in[0.2,0.5]$ and $d_{\mathrm{SB}} / L \in[0.08,0.5]$. We find that the distributions of $\ln \left(m_{i} / m_{\nu}\right)$ remain qualitatively the same as in the last row of Fig. 19. It is only when $d_{\mathrm{SB}}$ is much smaller than $d_{N}$ that there is a qualitative difference; then the Majorana mass matrix of right-handed neutrinos has hierarchical eigenvalues, and lighter right-handed neutrinos give rise to enhanced values of $m_{2} / m_{\nu}$, erasing the sharp cutoff in the distribution of $\ln \left(m_{2} / m_{\nu}\right)$.

\section{Neutrino mass hierarchy in Gaussian landscapes}

It is a robust consequence of Gaussian landscapes on any geometry that the seesaw masses are hierarchical. If the 
right-handed neutrinos have localized wave functions, then the neutrino Yukawa matrix obtains statistically hierarchical mass eigenvalues just like in the analysis of Secs. II and III. On the other hand, if the wave functions are not particularly localized over the extra dimension(s), then the Yukawa matrix always has small eigenvalues due to rank reduction. Because of the absence of correlation between the Majorana mass terms of right-handed neutrinos and the neutrino Yukawa couplings, the hierarchical structure of the neutrino Yukawa matrix remains in the seesaw mass eigenvalues. As a consequence, an inverted hierarchy is unlikely. In addition, it is likely that $m_{1} \ll m_{2}$ and

$$
\frac{m_{2}}{m_{3}} \simeq \sqrt{\frac{\Delta m_{\odot}^{2}}{\Delta m_{\mathrm{atm}}^{2}}} .
$$

Although Gaussian landscapes predict that the seesaw masses are hierarchical on average, the distribution of mass ratios from Gaussian landscapes are qualitatively different, depending on the choice of the width parameters $d_{N}$ and $d_{\mathrm{SB}}$. Consider the distribution of $m_{2} / m_{3}$ for various choices of these width parameters, displayed in Fig. 20. When neither the right-handed neutrino nor the symmetrybreaking wave function(s) are localized, the distribution of $m_{2} / m_{3}$ is sharply cutoff at around $10^{-2}$, seen in Figs 20(d) and 20(e). In this case there is no chance to accommodate the observed value,

$$
\sqrt{\frac{\Delta m_{\odot}^{2}}{\Delta m_{\mathrm{atm}}^{2}}} \simeq(1.6--2.0) \times 10^{-1} .
$$

If $d_{N}$ is large and the wave function $\varphi^{\mathrm{SB}}$ is strongly localized in the extra dimension(s), then the distribution of $m_{2} / m_{3}$ has a tail extending upward as in Fig. 20(f). This is

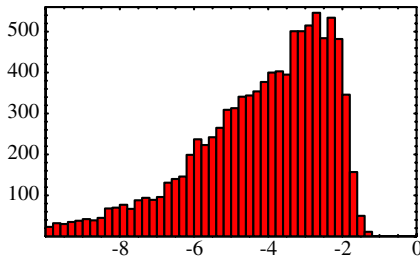

(a)

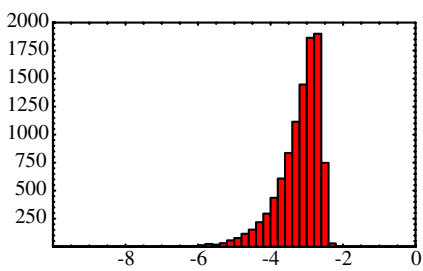

(e)

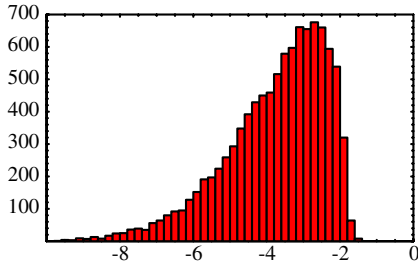

(b)

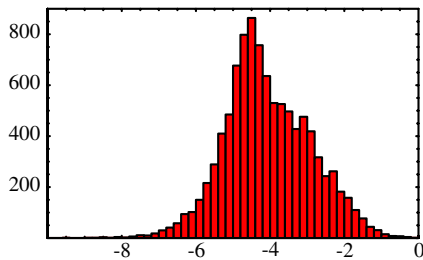

(f)

because of the previously mentioned enhancement in the seesaw mass matrix - due to small eigenvalues in the Majorana mass terms of the right-handed neutrinoswhich erases the sharp upper bound on $\lambda_{2}^{\nu}$. The distribution in Fig. 20(f) barely covers the observed value (87), but the width parameters may be chosen a little differently to provide better coverage in the tail of the distribution. Yet even in this scenario, the distribution of $m_{2} / m_{3}$ is peaked at a value much smaller than the observed value (87). If environmental selection factors (such as those associated with leptogenesis) and weight factors associated with cosmological evolution are in favor of such an outlier, then this scenario may be acceptable. Otherwise, one should conclude that this scenario is not a good approximation to the actual landscape governing the flavor structure of the multiverse.

It is left to consider localized wave functions for the right-handed neutrinos. The approximate distribution of $m_{2} / m_{3}$ is derived analytically in this scenario, and plotted in the right column of Fig. 18. Regardless of the geometry this distribution covers a broad logarithmic range, with the greatest weight toward the largest value, $m_{2} / m_{3} \sim 1$. These analytical distributions capture the qualitative features of Figs. 20(a), 20(b), and 20(g) very well, with one important difference: the distributions in the numerical results fall off to almost zero for $m_{2} / m_{3} \gtrsim 10^{-2}-10^{-1}$. This behavior of the numerical results is presumably due to the diagonalization effect, a phenomenon first mentioned in Sec. III B and subsequently referred to with regard to Figs. 5, 11, 14, 16, and 17. This reduced weight in the probability distribution functions at $m_{2} / m_{3} \gtrsim 10^{-1}$ means that this scenario is also in conflict with the observation (87).

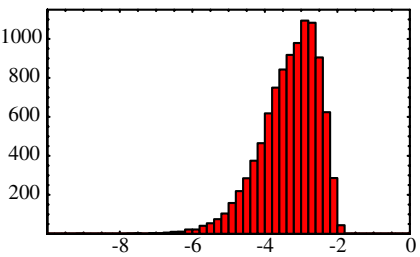

(c)

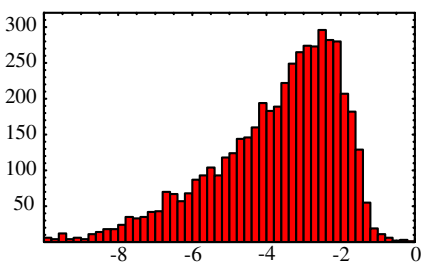

(g)

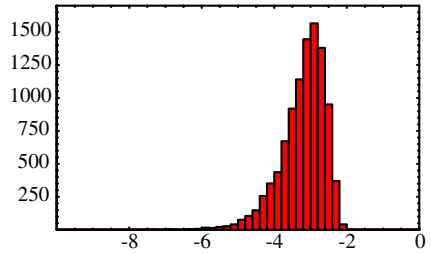

(d)

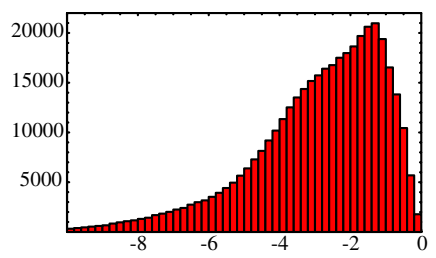

(h)

FIG. 20 (color online). Distributions of $\log _{10}\left(m_{2} / m_{3}\right)$ for various choices of parameters. The Gaussian landscape on $S^{1}$ was used for all simulations except (g), for which $T^{2}$ was used. In (f), $d_{\mathrm{SB}}$ is chosen to be very small so that $d_{H}^{4} / d_{\mathrm{SB}}^{2}$ is large, while (h) involves complex Gaussian profiles (see Sec. VID). Unless otherwise specified, $d_{\mathbf{1 0}} / L=d_{H} / L=0.08$ and $d_{\overline{5}} / L=0.3$. (a): $d_{N} / L=d_{\mathrm{SB}} / L=0.08$ (b): $d_{N} / L=d_{\mathrm{SB}} / L=0.10$ (c): $d_{N} / L=d_{\mathrm{SB}} / L=0.15$ (d): $d_{N} / L=d_{\mathrm{SB}} / L=0.2 \quad(\mathrm{e}): d_{N} / L=$ $d_{\mathrm{SB}} / L=0.3 \quad$ (f): $\quad d_{N} / L=0.3, \quad d_{\mathrm{SB}} / L=0.02, \quad d_{H} / L=0.1 \quad(\mathrm{~g}): \quad d_{N} / L=d_{\mathrm{SB}} / L=d_{H} / L=d_{10} / L=0.1, \quad$ on $\quad T^{2}$ (h): $d_{N} / L=d_{\mathrm{SB}} / L=0.08, r=3.0$. 

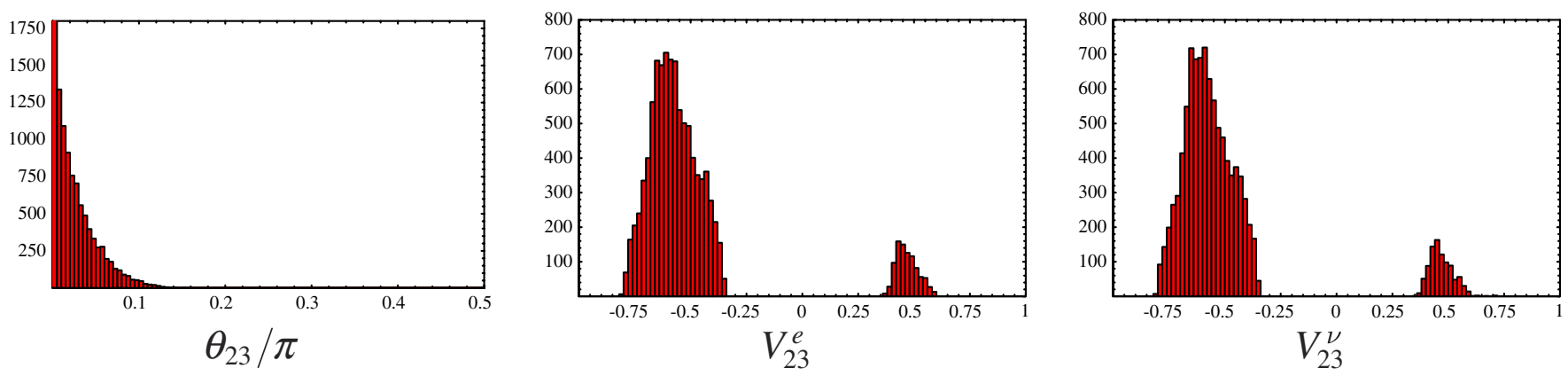

FIG. 21 (color online). The distributions of the PMNS mixing angle $\theta_{23}$, the $(2,3)$ element of the diagonalization matrix $V^{e}$, and that element of $V^{\nu}$. The distributions result from a numerical simulation of the Gaussian landscape on $S^{1}$ with $d / L=0.08$ for all fields except $d_{\overline{5}} / L=0.3$.

There is an important caveat, however, which is that the diagonalization effect changes as the real-valued matrices that we have dealt with so far are generalized to complexvalued matrices. See the appendix of [12], for example. Figure 20(h) is based on an extended version of the Gaussian landscape on $S^{1}$ (presented in Sec. VID) that includes complex phases. The diagonalization effect clearly has much less impact on the distribution function of $m_{2} / m_{3}$, and this distribution extends almost all the way up to $m_{2} / m_{3} \simeq 1$. Thus we find the scenario with localized wave functions for the right-handed neutrinos is compatible with the large value observed for $\Delta m_{\odot}^{2} / \Delta m_{\mathrm{atm}}^{2}$, assuming the Gaussian landscape contains complex phases.

\section{Mixing angles and $C P$ phases}

We introduced nonlocalized wave functions for the fields in $\overline{\mathbf{5}}$ because we expected this to result in large leptonic mixing angles. However, numerical simulation reveals that the mixing angles, particularly $\theta_{23}$, are still very small, as can be seen in the left panel of Fig. 21. The figure presented here is a simulation using small $d_{N} / L$ and $d_{\mathrm{SB}} / L$; but the $\theta_{23}$ distribution is confined near $\theta_{23} \sim 0$ no matter how small or large these parameters are taken to be.

The distributions of $V_{23}^{e}$ and $V_{23}^{\nu}$ in Fig. 21 explain why, contrary to our expectation, we failed to get large $\theta_{23}$. A $\mathrm{SO}(3)$ matrix $V^{e}$ acts on the left-handed charged leptons $e_{L i} \subset l_{i}$ when diagonalizing the charged-lepton Yukawa matrix $\lambda_{a i}^{e}$, and $V^{\nu}$ acts on the left-handed neutrinos $\nu_{L i} \subset$ $l_{i}$ when diagonalizing the seesaw mass matrix $C_{i j} / M$. Their $(2,3)$ components are large, just as we expected. Yet the mixing angle of atmospheric neutrino oscillation, the $(2,3)$ component of $U=\left(V^{e}\right)^{-1} V^{\nu}$, is small due to a precise cancellation between the large $V_{23}^{e}$ and $V_{23}^{\nu}$. This cancellation is not a new phenomenon. The democratic mass matrix ansatz, originally applied to the quark sector, yields small mixing angles through this precise cancellation.

To obtain large mixing angles, some modification is needed to prevent this cancellation. We replace the realpositive valued Gaussian wave functions in (20) by
Gaussian wave functions with a winding complex phase ${ }^{21}$ :

$$
\varphi\left(y ; y_{0}\right) \propto e^{-(1+r i)\left(\left(y-y_{0}\right)^{2} / 2 d^{2}\right)},
$$

introducing a new parameter $r$. For simplicity we use the same value of $r$ for all of the wave functions in the overlap integrations, $\varphi^{q, \bar{u}, \bar{d}, l, \bar{e}, \bar{\nu}, S B, h, h^{*}}$. The wave function of $h^{*}$ does not have to be the complex conjugate of that of $h$, since, for example, in the framework of Sec. VII these wave functions are not just scalars but have a more complicated internal structure. ${ }^{22}$

As seen in Fig. 22, using this form of wave function makes $V_{23}^{e}$ and $V_{23}^{\nu}$ complex, and their phases decrease the likelihood of a precise cancellation in $\theta_{23}$. Furthermore, these complex Gaussian wave functions introduce $C P$ violation into the flavor physics. Meanwhile, this introduction of complex phases has little effect on the distributions of charged fermion mass eigenvalues. This can be understood analytically by tracing the discussion in the preceding sections with the wave function (88). We also confirmed this result by running numerical simulations. The diagonalization effect is not taken into account in the theoretical arguments, but in practice this matters only for the $m_{2} / m_{3}$ neutrino mass distribution, and we have seen that this improves the agreement with observation.

\footnotetext{
${ }^{21}$ This form for the wave function is not without motivation, c.f. the discussion surrounding (154). However, we use this wave function only as one of the simplest means to introduce complex phases into Gaussian landscapes. There are more complicated, more sophisticated, and possibly better justified ways to introduce complex phases into Gaussian landscapes, or landscapes based on extra dimensions in general. It would be interesting to explore such models that include complex phases to study the correlation between the induced $C P$ violation and the distribution of other observables. However this subject is beyond the scope of this paper. We restrict attention to (88) and find that even this simple model is sufficient to obtain large leptonic mixing angles, when $d_{\overline{5}}$ is large.

${ }^{22}$ We have checked that one can obtain results very similar to those presented in this section, but taking $\varphi^{h}=\left(\varphi^{h^{*}}\right)^{*}$, if one also allows for nonuniversal values of $r$.
} 

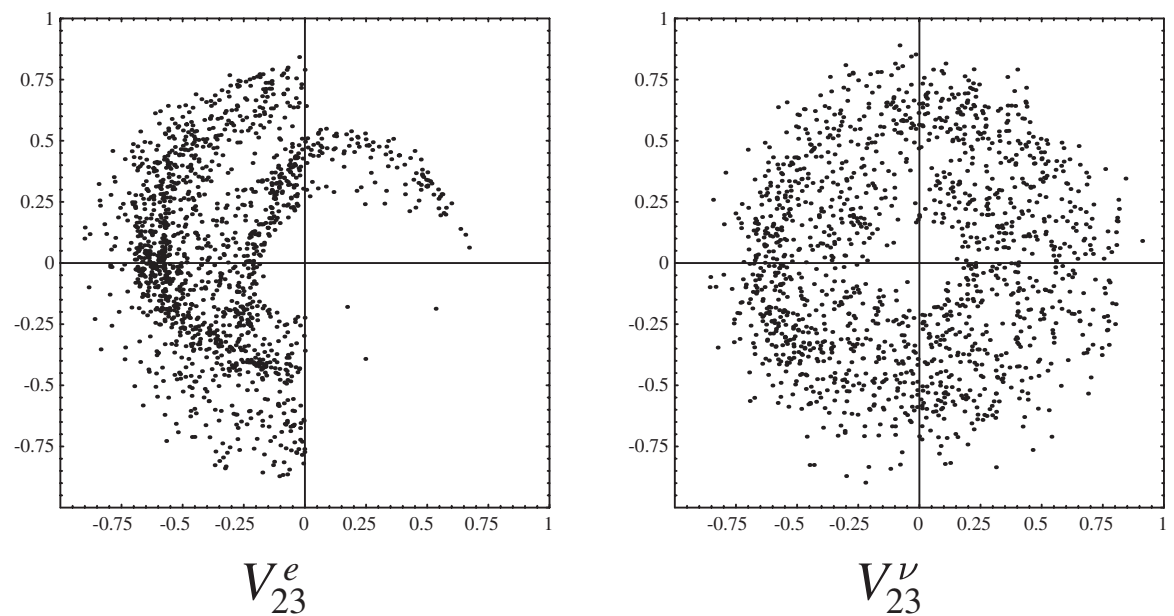

FIG. 22. Distribution of the $(2,3)$ component of the diagonalization matrices $V^{e}$ and $V^{\nu}$ in the complex plane. The simulation uses the Gaussian landscape on $S^{1}$, with $d / L=0.08$ for all fields except $d_{5} / L=0.3$; while $r=3$. The distributions of $\left|V_{23}^{e}\right|$ and $\left|V_{23}^{\nu}\right|$ remain much the same as in Fig. 21, but the phase angle prevents precise cancellations in $\theta_{23}$.

On the other hand, introducing complex phases dramatically changes the distributions of mixing angles. Figure 23 shows the distributions of the three leptonic mixing angles resulting from a numerical simulation. We see that the mixing angles of solar and atmospheric neutrino oscillations, $\theta_{12}$ and $\theta_{23}$, are likely to be any value between 0 and $\pi / 2$. Distributions of the oscillation amplitudes $\sin \left(2 \theta_{i j}\right)$ are almost flat for both of these mixing angles. Peaks at maximal mixing come from the Jacobian [12]. Comparing the distributions of $\theta_{23}$ between Figs. 21 and 23, we see that the introduction of complex phases (and hence $C P$ violation) is an essential ingredient in obtaining large (and even maximal) mixing angles in neutrino oscillations.

The mixing angle $\theta_{13}$ can also be of order unity, which is similar to the situation in neutrino anarchy $[8,12]$. In the $\mathrm{U}(3)$-invariant measure to be expected in neutrino anarchy, however, the distribution function of $\sin \left(2 \theta_{13}\right)$ is peaked at $\sin \left(2 \theta_{13}\right)=1$, and vanishes at $\sin \left(2 \theta_{13}\right)=0$. Thus all three mixing angles in the lepton sector are likely to be large. In particular, according to neutrino anarchy using the $\mathrm{U}(3)$-invariant measure, about $60 \%-90 \%$ of the statistics are already excluded by the current experimental limit on $\theta_{13}[8,12]$. In the Gaussian landscape on $S^{1}$, on the other hand, the $\theta_{13}$ distribution is weighted more toward $\sin \left(2 \theta_{13}\right)=0$. As we see in Sec. VIE, only about $30 \%$ of the statistics have been excluded.

For comparison, the distributions of the quark mixing angles are also simulated in the same Gaussian landscape and are presented in Fig. 24. We see that the complexvalued Gaussian profiles maintain small mixing angles when the overlap integrals involve narrow Gaussian widths for 10's and the Higgs boson. Interestingly, simply choosing different widths for particles in the $\overline{5}$ and $\mathbf{1 0}$ representations, in the presence of large $C P$-violating phases, allows for very different flavor structures between the quark and lepton sectors. Thus pursuing a microscopic description behind the statistical distributions of Yukawa couplings has enabled us to go beyond the results of $[8,9]$.

The distributions of the $C P$-violating phases are also shown in Fig. 25. Our parametrization of the CKM and leptonic mixing matrices $V$ and $U$ is quite standard:

$$
\begin{gathered}
V=\left(\begin{array}{ccc}
c_{12} c_{13} & s_{12} c_{13} & s_{13} e^{-i \delta_{\mathrm{CKM}}} \\
-s_{12} c_{23}-c_{12} s_{23} s_{13} e^{i \delta_{\mathrm{CKM}}} & c_{12} c_{23}-s_{12} s_{23} s_{13} e^{i \delta_{\mathrm{CKM}}} & s_{23} c_{13} \\
s_{12} s_{23}-c_{12} c_{23} s_{13} e^{i \delta_{\mathrm{CKM}}} & -c_{12} s_{23}-s_{12} c_{23} s_{13} e^{i \delta_{\mathrm{CKM}}} & c_{23} c_{13}
\end{array}\right), \\
U=\left(\begin{array}{cccc}
c_{12} c_{13} & s_{12} c_{13} & s_{13} e^{-i \delta_{\nu}} \\
-s_{12} c_{23}-c_{12} s_{23} s_{13} e^{i \delta_{\nu}} & c_{12} c_{23}-s_{12} s_{23} s_{13} e^{i \delta_{\nu}} & s_{23} c_{13} \\
s_{12} s_{23}-c_{12} c_{23} s_{13} e^{i \delta_{\nu}} & -c_{12} s_{23}-s_{12} c_{23} s_{13} e^{i \delta_{\nu}} & c_{23} c_{13}
\end{array}\right)\left(\begin{array}{ccc}
e^{i \alpha_{1} / 2} & & \\
& e^{i \alpha_{2} / 2} & \\
& & 1
\end{array}\right),
\end{gathered}
$$

where $s_{i j} \equiv \sin \theta_{i j}$ and $c_{i j} \equiv \cos \theta_{i j}$. The Majorana mass phases of the neutrinos, $\alpha_{1,2}=\arg \left(m_{1,2} / m_{3}\right)$, have almost flat distributions. On the other hand, the distributions of the two other $C P$ phases, $\delta_{\mathrm{CKM}}$ and $\delta_{\nu}$, have peaks at $\delta \sim 0$ on top of otherwise flat distributions. We have not studied where this structure comes from. The scatter plot of $V_{23}^{e}$ in Fig. 22 reveals some structure - the scatter is dense in some places and thin in others - and the peaks at $\delta=0$ 

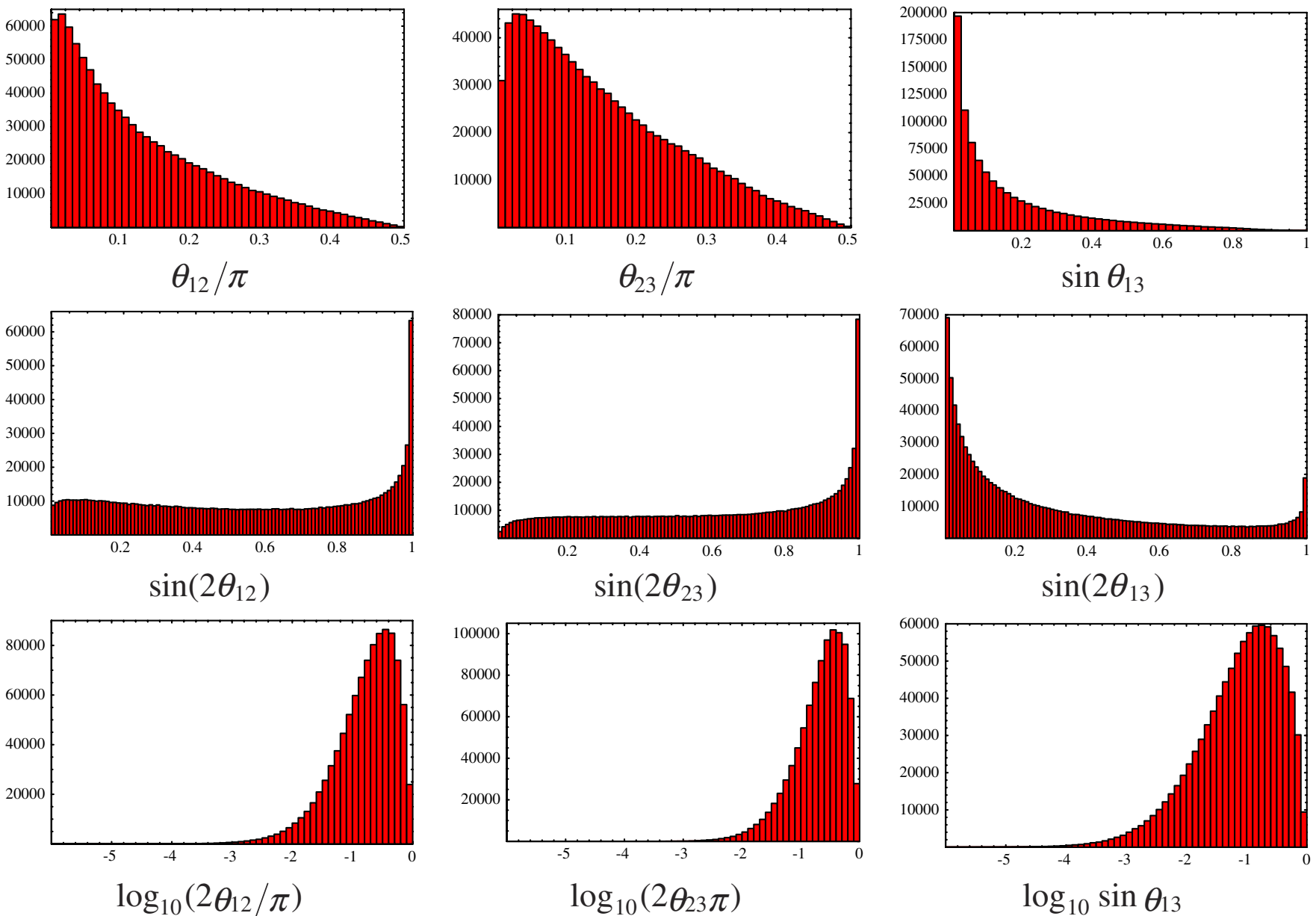

FIG. 23 (color online). Distribution of the leptonic mixing angles in the Gaussian landscape on $S^{1}$, with complex-valued wave functions with $r=3$ and $d / L=0.08$ for all fields except $d_{\overline{5}} / L=0.3$.

in the $C P$ phase distributions may have something to do with this structure, which presumably originates from the specific form of the wave function (88). If this guess is correct, then the peak plus flat structure in the $C P$ phase distributions is likely to be an artifact of the particular way in which we have introduced complex phases into this landscape. It would be interesting to see how the $C P$ phase distribution changes when complex phases are introduced in different ways, but this subject is beyond the scope of this paper. Although we expect that the peak structure may deform, disappear, or become less significant, we consider it unlikely that the flat part of distribution would disappear and that $C P$ phases would be predicted to be very small. If this expectation is correct, then these landscapes are consistent with the measured value of the $C P$ phase in the quark sector.

\section{E. Conditional probabilities}

The neutrino sector has three observables yet to be measured, $\sin \theta_{13}, \delta_{\nu}$, and $m_{\beta \beta}$. It would be interesting if landscape approaches to understanding flavor could make predictions for these observables. We have developed our Gaussian landscapes so that all the observables measured in this universe are not too atypical. In this section, we use the Gaussian landscape on $S^{1}$, with the set of parameters $r=3, \quad d_{H} / L=d_{10} / L=d_{N} / L=d_{S B} / L=0.08, \quad$ and $d_{\overline{5}} / L=0.3$, as an initial example to explore what kind of predictions can be obtained from this approach. ${ }^{23}$

The mixing angle $\theta_{13}$ is typically of order unity, and there is no reason to expect from this Gaussian landscape that it is very small. Indeed, the current experimental limit has excluded a significant fraction of the ensemble of

\footnotetext{
${ }^{23}$ This Gaussian landscape has 19 scanning parameters, corresponding to the center coordinates of the wave functions of the quarks, leptons, Higgs boson, and symmetry-breaking field(s) $\varphi^{\mathrm{SB}}$. Meanwhile, this landscape now predicts distributions for all 22 flavor parameters. Although this implies that the landscape makes three precise (zero-width) predictions; this is a result of our arbitrary choice to consider parameters such as $g$, $L / d_{\overline{\mathbf{5}}, \mathbf{1 0}, N, \mathrm{SB}}$, and $r$ as fixed. Specifically, these predictions disappear when these parameters also have probability distributions around the values we fixed. Therefore we do not pursue the possibility of a truly predictive Gaussian landscape.
} 

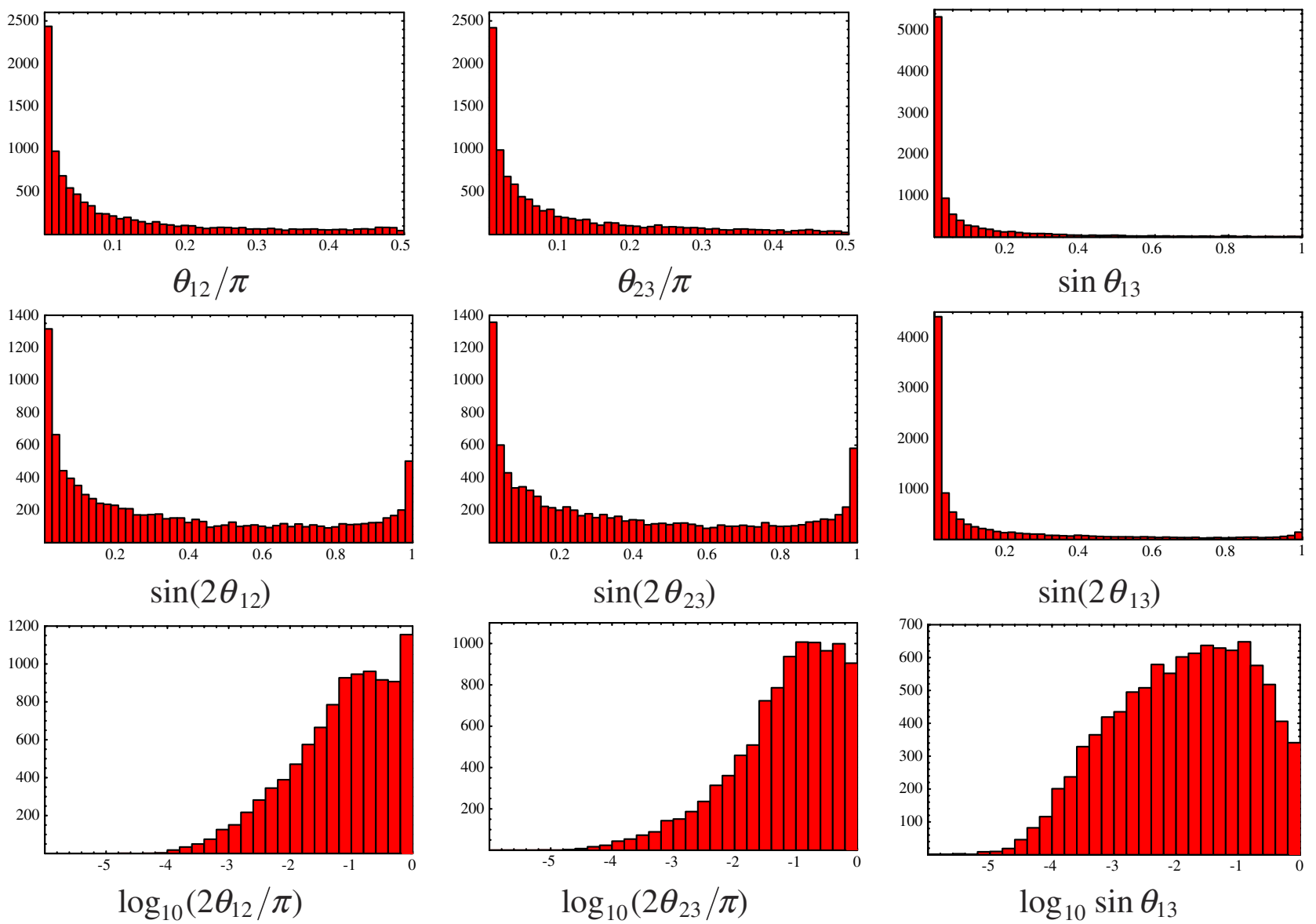

FIG. 24 (color online). Distribution of the quark mixing angles in the Gaussian landscape on $S^{1}$, with complex-valued wave functions with $r=3.0, d_{10} / L=d_{H} / L=0.08$, and $d_{5} / L=0.3$.
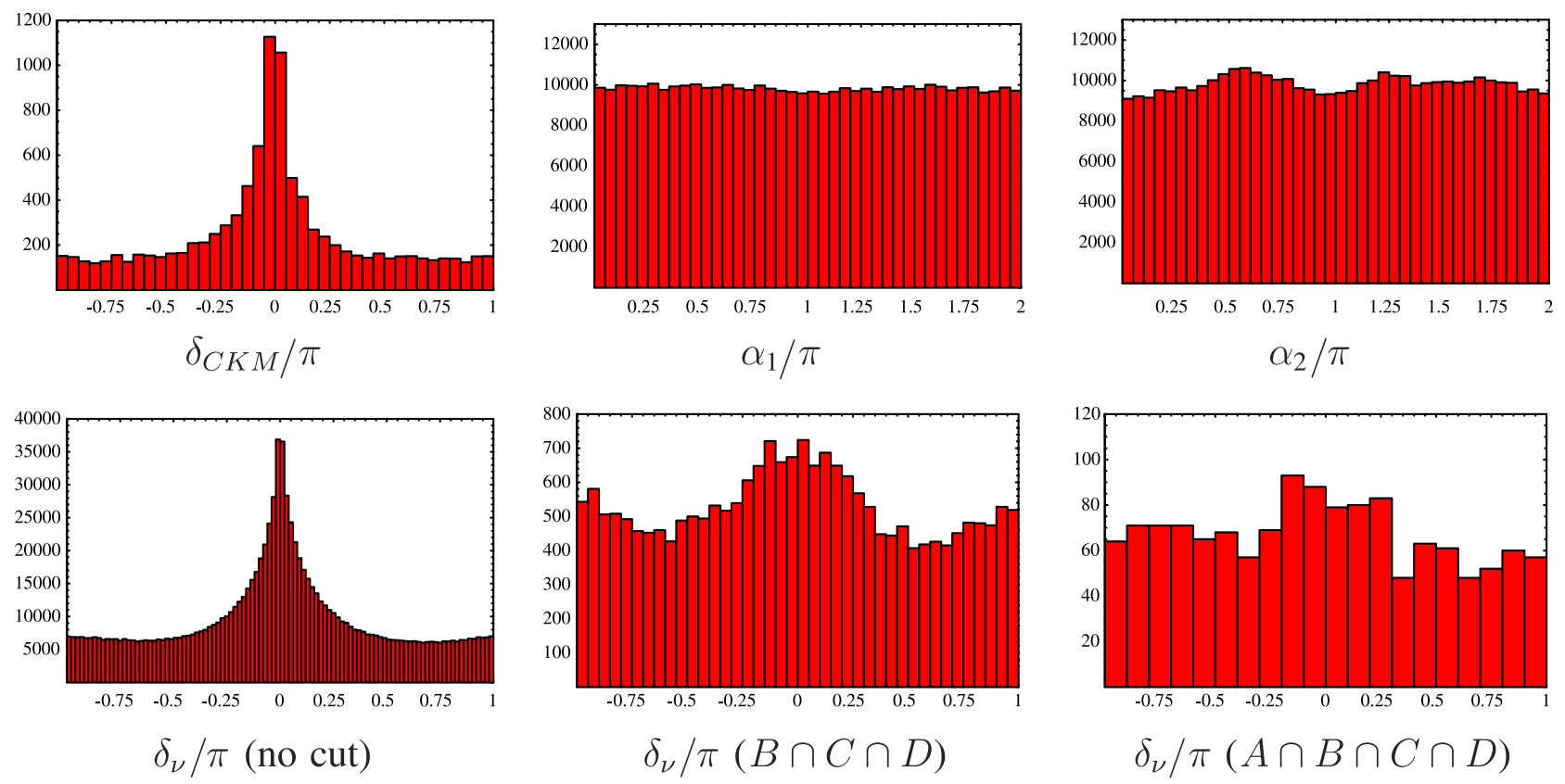

FIG. 25 (color online). Distribution of the $C P$ phases in the quark and lepton sectors, based on the Gaussian landscape on $S^{1}$ with $r=3.0, d / L=0.08$ for all fields except $d_{\overline{5}} / L=0.3$. 
vacua:

$$
P\left(\sin \theta_{13}>0.18\right)=33 \% .
$$

Nevertheless, the experimental limit is not very strong and so a significant fraction of vacua- $67 \%$ of the total ensemble in this Gaussian landscape — sits within the experimental bound. On the other hand, future experiments are expected to have a sensitivity down to about $\theta_{13} \sim 10^{-2}$. The probability that $\theta_{13}$ is too small to be measured by such future experiments is rather low within this Gaussian landscape; we find

$$
P\left(\sin \theta_{13}<10^{-2}\right)=12 \% .
$$

The second yet-to-be-measured parameter is the $C P$ phase of neutrino oscillations. The probability distribution of this is displayed in the bottom left panel of Fig. 25. Finally, neutrinoless double beta decay measures $\left|m_{\beta \beta}\right|$, where

$$
\begin{gathered}
m_{\beta \beta}=\sum_{i=1}^{3} U_{e i}^{2} m_{i} \equiv m_{2} U_{e 2}^{2}\left[1+\left(\frac{U_{e 3}}{U_{e 2}}\right)^{2} \frac{m_{3}}{m_{2}}+\Delta R\right] \\
\Delta R=\left(\frac{U_{e 1}}{U_{e 2}}\right)^{2} \frac{m_{1}}{m_{2}}
\end{gathered}
$$

The second term of (93) is roughly

$$
\left|\frac{U_{e 3}}{U_{e 2}}\right|^{2}\left|\frac{m_{3}}{m_{2}}\right| \sim 0.57\left(\frac{\sin \theta_{13}}{0.18}\right)^{2} \sim 0.18\left(\frac{\sin \theta_{13}}{0.10}\right)^{2},
$$

after using (86) and (87). Once $\sin \theta_{13}$ is measured, the second term of (93) can be estimated. Yet we still need to know the last term, $\Delta R$, to make a prediction for $\left|m_{\beta \beta}\right|$. The Gaussian landscape distribution of $|\Delta R|$ is found in the top right panel of Fig. 26.

We can ask more specific questions to further constrain the probability distributions of these three observables in universes like ours. That is, when making predictions for future measurements, we can condition the probability distributions based on quantities that we have already measured, as opposed to using the a priori probability distributions in the landscape. However, if we were to impose the current experimental limit on all lepton flavor parameters, an enormous numerical simulation would be required, since most of the simulated universes would not pass the cut. This does not imply that our universe is very atypical, only that the present experimental error bars are small. Therefore, for practical reasons we employ much looser experimental cuts ${ }^{24}$

\footnotetext{
${ }^{24}$ One might consider these cuts as a tool to study the effects of possible cosmological or environmental selection in the multiverse, just like a large top-Yukawa cut (43) was employed in Sec. III in an attempt to study the impact of environmental selection in favor of a large top-Yukawa coupling. Here, however, we know of no evidence that the weight factors are in favor of the range of parameters selected by the cut conditions $A-D$.
}

$$
\begin{gathered}
A: 10^{-2}<\Delta m_{\odot}^{2} / \Delta m_{\mathrm{atm}}^{2}<10^{-1}, \\
B: \sin ^{2}\left(2 \theta_{12}\right)>0.7, \\
C: \sin ^{2}\left(2 \theta_{23}\right)>0.8, \\
D: \sin \theta_{13}<0.18,
\end{gathered}
$$

and study whether these cuts influence the distributions of $\theta_{13}, \Delta R$, and $\delta_{\nu}$.

Table II shows how the probability to measure $\sin \theta_{13}>$ $3 \times 10^{-2}$ changes depending on whether or not the cut conditions $A-C$ are imposed. The loose cuts $A-C$ have a significant impact on the prediction. Among all the samples that are consistent with the current experimental limit on $\sin \theta_{13}, 40 \%$ of them are below $3 \times 10^{-2}$. However, after imposing various other experimental constraints, $A-C$, only a few percent have $\sin \theta_{13}<3 \times 10^{-2}$. Therefore, for the wave function parameter choices used in this section, the Gaussian landscape on $S^{1}$ predicts a very high probability of measuring $\theta_{13}$ in future experiments in our universe.

When $\sin \theta_{13}$ is large, there is a good chance to observe $C P$ violation in neutrino oscillation experiments. The $a$ priori vacuum statistics for this parameter are peaked at $\delta_{\nu}=0$ (bottom left panel of Fig. 25). However the distribution of $\delta_{\nu}$ changes as some of the cuts $A-D$ are imposed. The impact of these changes is clearly visible when the mixing angles of both the solar and atmospheric neutrino oscillations are required to be large; then the peak of the $C P$ phase distribution is significantly reduced (bottom middle panel of Fig. 25). This is reasonable, since complex phases play a crucial role in avoiding cancellation between large mixing angles in $V^{e}$ and $V^{\nu}$. In scatter plots in the $\sin ^{2}\left(2 \theta_{13}\right)-\delta_{\nu}$ plane (Fig. 26), we see that the distribution of the $C P$ phase becomes uniform after cuts $A-D$ are imposed. Therefore, after conditioning distributions based on our loose "experimental cuts," both $\sin \theta_{13}$ and the $C P$ phase become more likely to be larger, and hence more likely to be discovered in future experiments.

The loose cuts $A-D$ also have some impact on the distribution of $|\Delta R|$ (Fig. 26). The condition $B$ alone, requiring $\left|U_{e 2}\right|$ be large, removes almost all the distribution $\log _{10}|\Delta R|>0$ as well. Examining the samples that pass all cuts $A-D$, we find that

$$
\begin{gathered}
P(|\Delta R|>1)=(0.3 \pm 0.2) \%, \\
P(|\Delta R|>0.1)=(9 \pm 1) \%,
\end{gathered}
$$

where the uncertainty comes from limited statistics in the numerical simulation. Therefore, it is very unlikely that $\Delta R$ is so large as to be significant next to the first term $m_{2} U_{e 2}^{2}$ in $m_{\beta \beta}$. Hence this landscape predicts $m_{\beta \beta} \simeq$ $m_{2} U_{e 2}^{2}+m_{3} U_{e 3}^{2}$ and 


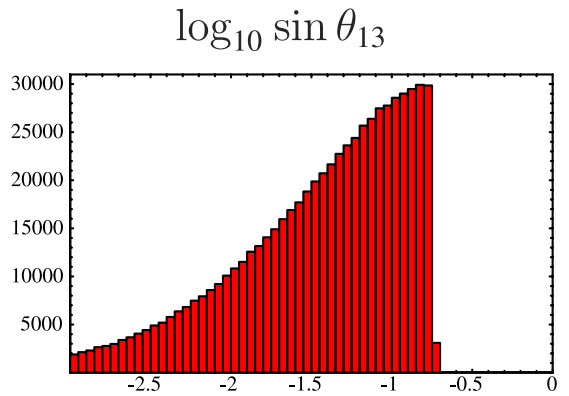

$(D)$
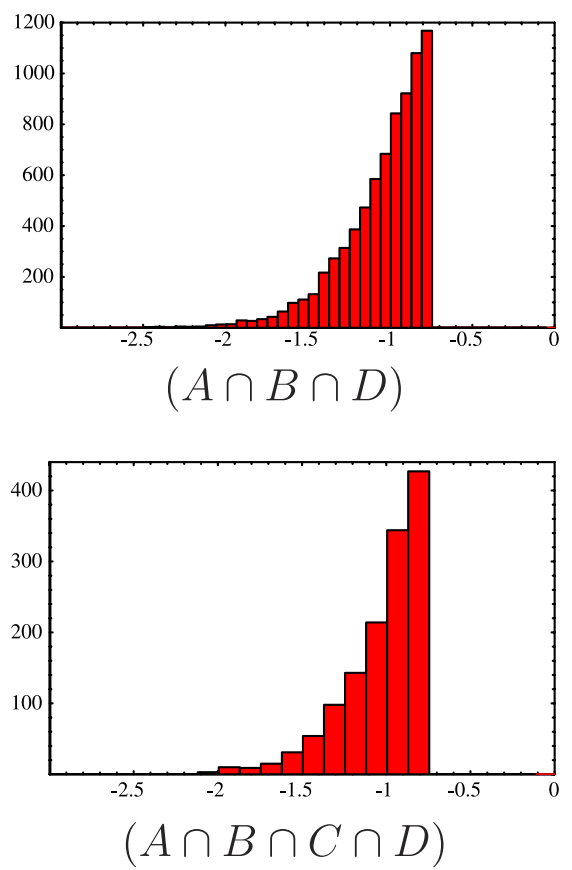

$$
\log _{10} \sin ^{2}\left(2 \theta_{13}\right)-\delta_{\nu} / \pi
$$

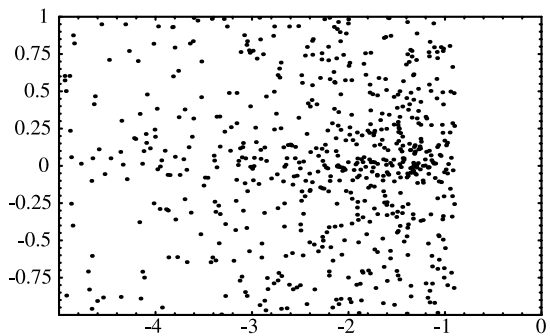

$(D)$

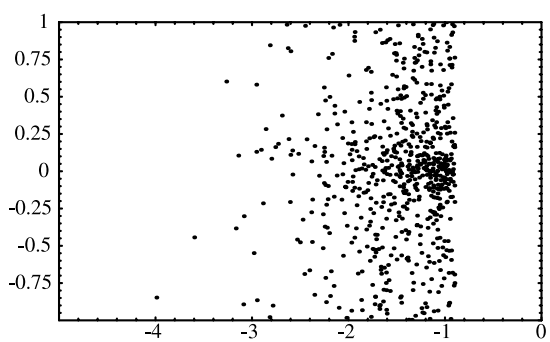

$(A \cap B \cap D)$

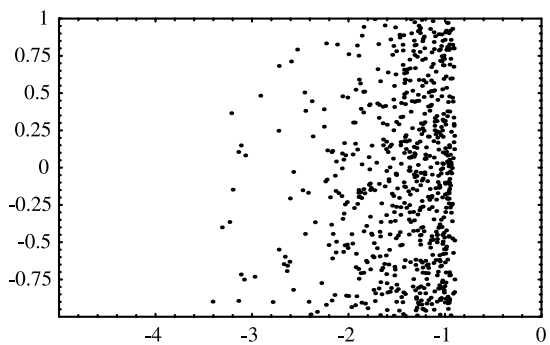

$(A \cap B \cap C \cap D)$ $\log _{10}|\Delta R|$

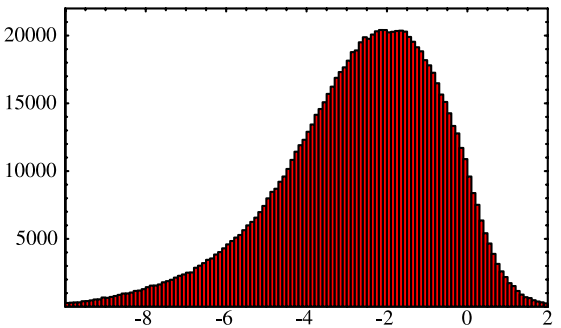

(no cut)

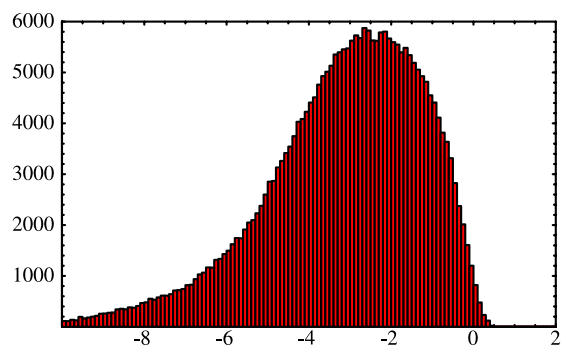

$(B)$

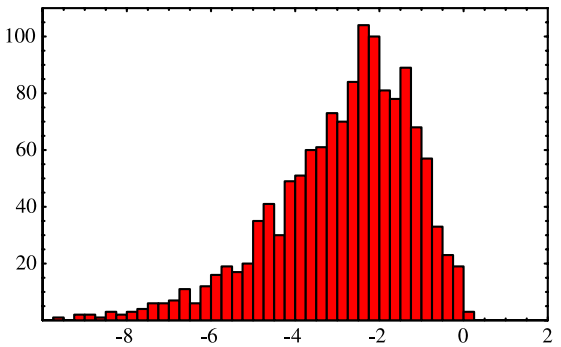

$(A \cap B \cap C \cap D)$

FIG. 26 (color online). Distributions of observables in the neutrino sector for different subsets of the total ensemble. The Gaussian landscape on $S^{1}$ is used for this simulation, with $r=3$ and $d / L=0.08$ for all fields except $d_{\mathbf{5}} / L=0.3$. In the $\log _{10} \sin ^{2}\left(2 \theta_{13}\right)-\delta_{\nu}$ scatter plots, the same number of points are displayed in all three figures.

$$
\begin{aligned}
\left|m_{2} U_{e 2}^{2}\right| & \times\left[1-0.18\left(\frac{\sin \theta_{13}}{0.10}\right)^{2}\right] \lesssim\left|m_{\beta \beta}\right| \lesssim\left|m_{2} U_{e 2}^{2}\right| \\
& \times\left[1+0.18\left(\frac{\sin \theta_{13}}{0.10}\right)^{2}\right],
\end{aligned}
$$

where $\left|m_{2} U_{e 2}^{2}\right| \simeq(2-3) \times 10^{-3} \mathrm{eV}$.

We have seen that the loose cuts $A-D$ have a significant impact on the distributions of $\sin \theta_{13}, \delta_{\nu}$, and $|\Delta R|$. This study gives us a feeling for how much landscape predictions can change when various weight factors multiply the simple vacuum statistics, or when various experimental measurements are used to condition predictions based on what we know about our universe. Details about these predictions will depend on the specific weight factors or how severely the experimental cuts are imposed. However, as we have seen, the impact of these cuts can be understood qualitatively, and we expect similar results for similar weights or cuts.

Although the width parameters of $\mathbf{1 0}, \overline{\mathbf{5}}$, and the Higgs boson have been optimized to fit the observed data, there is

TABLE II. Probability to measure $P\left(\sin \theta_{13}>3 \times 10^{-2}\right)$ under various cuts. Up to $\pm 1 \%$ uncertainties arise from the limited statistics gathered in the numerical simulation. We use the Gaussian landscape on $S^{1}$ with $r=3$ and $d / L=0.08$ for all fields except $d_{\overline{5}} / L=0.3$.

\begin{tabular}{lccccccc}
\hline \hline$D$ & $A \cap D$ & $B \cap D$ & $C \cap D$ & $A \cap B \cap D$ & $A \cap C \cap D$ & $B \cap C \cap D$ & $A \cap B \cap C \cap D$ \\
\hline $61 \%$ & $75 \%$ & $78 \%$ & $77 \%$ & $94-95 \%$ & $79 \%$ & $87 \%$ & $96 \pm 1 \%$ \\
\hline \hline
\end{tabular}


still much room left to change $d_{N}$ and $d_{\mathrm{SB}}$ (or even $r$ or how complex phases are introduced into the Gaussian landscape). If $d_{N}$ and/or $d_{\mathrm{SB}}$ were chosen slightly larger, then the distribution of $m_{2} / m_{3}$ covers a smaller range, and the cut $A$ would have less impact while the other three cuts on the mixing angles would remain as important. Since it was cuts $B \cap C$ and $B$, respectively, that had impacts on the distributions of $\delta_{\nu}$ and $|\Delta R|$, the "predictions" on $\delta_{\nu}$ and $|\Delta R|$ would not be affected very much. On the other hand, since the $A \cap B$ cut was important in dragging the distribution of $\sin \theta_{13}$ upward, the distribution of $\sin \theta_{13}$ may shift downward for a slightly larger $d_{N}$ or $d_{\mathrm{SB}}$. Thus the predictions in this section should be interpreted carefully, with these and other caveats ${ }^{25}$ in mind.

\section{YUKAWA COUPLINGS FROM SUPER YANG-MILLS INTERACTIONS}

In the preceding sections, we introduced a number of toy models, collectively termed Gaussian landscapes, to generate statistical ensembles of Yukawa matrices. The pairing and generation structures of the CKM matrix along with the large mixing angles of the lepton sector were successfully explained as random selections from these ensembles. The most crucial ingredient to these Gaussian landscapes was that Yukawa couplings are given by overlap integration of localized zero-mode wave functions on extra dimensions. The correlation necessary to account for generation structure arose because the wave functions of quark doublets and the Higgs are relevant for both the uptype and down-type Yukawa matrices. We also introduced a number of additional assumptions concerning which parameters are scanned and which are not. In this section we give greater theoretical consideration to these assumptions.

Consider a supersymmetric Yang-Mills theory on a higher-dimensional manifold. The internal space of $D$ extra dimensions is denoted $X_{D}$. The gauge group $G$ is chosen so that it contains a subgroup $H$ such as $\mathrm{SU}(3)_{C} \times$ $\mathrm{SU}(2)_{L} \times \mathrm{U}(1)_{Y}$ of the standard model or a unified gauge group such as $\mathrm{SU}(5)_{\mathrm{GUT}}$. The gauge fields $A_{m}$ of the theory may have nontrivial configurations on $X_{D}$ as long as they

\footnotetext{
${ }^{25}$ For example, landscapes of Yukawa couplings originating from super Yang-Mills theory in higher dimensions have basisindependent distributions, but Gaussian landscapes - simplified versions of the former-do not (see Sec. VII). This situation is compared to the basis-independent implementation of neutrino anarchy in [12] and the original implementation in [8]. The latter predicted anticorrelations between mixing angles in the lepton sector, but these angles are uncorrelated in the former. Thus some of the correlations in Gaussian landscapes may be spurious. Nevertheless, the disappearance of correlations in the basisindependent neutrino anarchy was related to invariance under the $\mathrm{U}(3)$ group. Unless there is a similar underlying symmetry, we expect that the probability distributions of Gaussian landscapes are not qualitatively different from those of higher-dimensional gauge theories.
}

are stable (and hence satisfy the equations of motion). Such a gauge-field configuration can break the underlying gauge symmetry $G$ down to $H$.

In this context the fields $A_{\mu}(\mu=0,1,2,3)$ in $\mathfrak{h}$ become the gauge fields of the standard model or the unified theory. Meanwhile, the Kaluza-Klein spectrum of $A_{m}(m=$ $5, \cdots, 4+D$ ) in $\mathfrak{g}$, under the gauge-field background, may have zero modes (i.e., massless modes), and such quantum fluctuations become scalar fields in the fourdimensional low-energy effective theory. Gauginos in $\mathfrak{g}$ may also have zero modes; since a spinor in a higherdimensional space is bi-spinor of the Lorentz group $\mathrm{SO}(3,1)$ and $\mathrm{SO}(D)$, and the zero modes of the Dirac equation on $X_{D}$ become massless fermions in the effective theory. These low-energy scalar and spinor fields may be charged under the standard model or unified theory gauge group, or they may be singlets under these gauge symmetries. This is worked out by decomposing the adjoint representation of $\mathfrak{g}$ into pieces irreducible under $H$. Charged fields arising in this way may well be identified with quarks, leptons, and the Higgs boson.

Fermion and scalar fields arising from the super YangMills fields in higher dimensions have Yukawa couplings in the low-energy effective theory. To see this more explicitly, consider the Lagrangian of a super Yang-Mills theory in higher dimensions:

$$
\begin{aligned}
\mathcal{L}_{4+D}= & -\frac{1}{4}\left[\frac{M_{*}^{D}}{g_{*}^{2}} \operatorname{tr}\left(F_{\mu \nu} F^{\mu \nu}\right)+2 \frac{M_{*}^{D}}{g_{*}^{2}} \operatorname{tr}\left(F_{\mu m} F^{\mu m}\right)\right. \\
& \left.+\frac{M_{*}^{D}}{g_{*}^{2}} \operatorname{tr}\left(\bar{\Psi} \Gamma^{\mu} \partial_{\mu} \Psi\right)+\cdots\right] .
\end{aligned}
$$

These three terms become the kinetic terms for the vector, scalar, and spinor fields in the effective theory. Meanwhile, the gauge coupling constant of the low-energy effective theory is

$$
\frac{1}{g_{\mathrm{eff}}^{2}}=\frac{M_{*}^{D} V}{g_{*}^{2}}
$$

where $V$ is the volume of the internal space. When the zero-mode wave functions $\varphi_{m}(y)$ and $\psi(y)$ are normalized such that

$$
M_{*}^{D} \int_{X_{D}} d^{D} y\left|\varphi_{m}\right|^{2}=1, \quad M_{*}^{D} \int_{X_{D}} d^{D} y|\psi|^{2}=1,
$$

then canonically normalized kinetic terms result when the low-energy fields are related to those in the higherdimensional theory by

$$
A_{m}(x, y)=g_{*} \varphi_{m}(y) \phi(x), \quad \Psi(x, y)=g_{*} \psi(y) \cdot \chi(x) .
$$


Then low-energy Yukawa couplings originate from the gauge interactions of gauginos

$$
\int_{X_{D}} d^{D} y \mathcal{L}_{4+D}=\int_{X_{D}} d^{D} y \frac{M_{*}^{D}}{4 g_{*}^{2}} \operatorname{tr}\left(\bar{\Psi} i \Gamma^{n}\left[A_{n}, \Psi\right]\right) \rightarrow \lambda \bar{\chi} \phi \chi,
$$

with the effective Yukawa coupling constants given by

$$
\lambda=\frac{M_{*}^{D} g_{*}}{4} \int_{X_{D}} d^{D} y \operatorname{tr}\left(\bar{\psi} i \gamma^{m}\left[\varphi_{m}, \psi\right]\right)
$$

Thus the low-energy Yukawa couplings are given by overlap integrations, just as in (22), where now $g_{*}$ is interpreted as the gauge coupling constant of the super Yang-Mills theory.

Having specified the origin of the quarks, leptons, and their Yukawa couplings, we know what the underlying gauge group $G$ has to be. For $H=\mathrm{SU}(5)_{\mathrm{GUT}}$, super Yang-Mills interactions of $G=E_{7}$ or $E_{8}$ give rise to all of the Yukawa couplings of the quarks, charged leptons, and neutrinos [19]. For the Pati-Salam group $H=$ $\mathrm{SU}(4)_{C} \times \mathrm{SU}(2)_{L} \times \mathrm{SU}(2)_{R}, \quad G=\mathrm{SU}(8) \quad$ is sufficient. When the heterotic $E_{8} \times E_{8}^{\prime}$ or $\mathrm{SO}(32)$ string theory is compactified on a real six-dimensional compact manifold, we obtain Yukawa couplings in this way; for example, with $G=E_{8}$ and a six-dimensional manifold $X_{D=6}$ for the $E_{8} \times$ $E_{8}^{\prime}$ string theory. In some compactifications of the type IIA string theory (or supergravity on 11 dimensions) and type IIB string theory (or F theory), the Yukawa couplings of quarks and leptons arise from open string interactions. For example in the type IIA string theory with an intersecting D6-D6 system, with quarks and leptons localized at the intersections of D6-branes, the Yukawa couplings are generated by spanning a world sheet to three intersection points with D6-branes connecting them. However, some of these compactifications can be understood as a certain limit of heterotic compactification; this is the essence of string duality. Thus the toy models that we use to calculate Yukawa couplings can capture certain aspects (and maybe all) of these compactifications as well. Therefore, the numerical analyses of this paper can be used to understand the statistics of flavor structure arising from a large region of the string theory landscape.

If quarks, leptons, and the Higgs boson originate from a super Yang-Mills theory of $G$ containing $H$, and the Yukawa couplings from the super Yang-Mills interaction of $G$, then we cannot arbitrarily assume the following:

(i) the shape of zero-mode wave functions - these must be solutions to the equations of motion.

(ii) the number of zero modes - this is determined by the topology of the geometry of the internal manifold $X_{D}$ and of the gauge-field configuration on $X_{D}$.

(iii) the number of independent parameters that freely scan - only moduli parameters, i.e. deformations of the manifold and gauge-field configuration that cost no energy, are scanned.

The possible restrictions above were ignored in the preceding sections, as we introduced assumptions instead. In this section we discuss when such assumptions are justified, when they are not, and when not what one should expect instead. In Secs. VII A and VII B we provide a pedagogical and bottom-up introduction to ideas that motivated us to assume Gaussian zero-mode wave functions with center coordinates that scan over the landscape. Most of the content of these sections is not particularly new, and the intended audience is nonstring theorists. We use the remainder of this section to describe what the toy models of Secs. III, V, and VI-i.e. Gaussian landscapes - mean in the context of the string theory landscape.

Before we proceed, let us comment on the basis independence of observables. The standard model has three independent fermions in a given representation, and the $3 \times 3$ Yukawa matrices transform under a group $\mathrm{U}(3)_{q} \times$ $\mathrm{U}(3)_{\bar{u}} \times \mathrm{U}(3)_{\bar{d}} \times \mathrm{U}(3)_{l} \times \mathrm{U}(3)_{\bar{e}}$ that preserves the canonical kinetic terms of the fermions. Observables such as mass eigenvalues and mixing angles also do not change under this transformation. Thus, any probability distribution of Yukawa matrices in a landscape-based theory should be either invariant under the U(3) transformations, or defined only for classes of Yukawa matrices equivalent under the $\mathrm{U}(3)$ transformations. This basis independence follows in landscapes that derive from super Yang-Mills interactions. Indeed, the zero modes-i.e. the solutions of massless Dirac equations in a given representation - form a vector space, the rank of which gives the number of generations. One should find an orthonormal basis of the vector space $\left\{\psi_{I}\right\}$, such that the metric of the kinetic term is given by

$$
M_{*}^{D} \int_{X_{D}} d^{D} y \psi_{I}^{*} \psi_{J}=\delta_{I J}
$$

and use the zero-mode wave functions of the basis vectors to calculate the elements of Yukawa matrices through

$$
\lambda_{I J}=\frac{M_{*}^{D} g_{*}}{4} \int_{X_{D}} d^{D} y \operatorname{tr}\left(\bar{\psi}_{I} i \gamma^{m}\left[\varphi_{m}, \psi_{J}\right]\right)
$$

The above U(3) transformations correspond to basis transformations of the vector space of zero modes. Here the $\mathrm{U}(3)$ basis transformations correspond to no more than choosing different descriptions of the same vacuum, ${ }^{26}$ and the actual statistical elements of the landscape correspond to vacua, not Yukawa matrices. Thus, any landscape

\footnotetext{
${ }^{26}$ Only the vector space composed of solutions to the zeromode equations of motion have these U(3) "symmetries;" the interaction terms - which are trilinear (not quadratic) in fields on a given background-do not respect these flavor symmetries at all.
} 
generating vacuum statistics through the overlap integrals of zero mode wave functions is basis independent. ${ }^{27}$

The Gaussian landscapes introduced in Secs. III, V, and VI are meant to be simplified versions of (subsets of) the landscape that derives from string compactification. Because of the way we have simplified the landscapescanning the center coordinates of zero modes completely randomly and independently - the basis independence of the landscape from string compactification is lost in our Gaussian landscapes. However, if the Gaussian landscapes are regarded as tools to acquire a better understanding of flavor physics in the landscape from string compactification - and this is certainly our attitude - then the lack of basis independence in Gaussian landscapes is itself not a major problem.

\section{A. Domain-wall fermion and $T^{2}$ compactification of field theories on six dimensions}

\section{Domain-wall fermion}

It is well known that a chiral fermion in a fourdimensional effective theory is localized at a domain wall in a five-dimensional theory [20]. Consider a fermion in five dimensions,

$d^{4} x d x_{5} \mathcal{L}_{5}=d^{4} x d x_{5}(\chi, \bar{\psi})\left[i\left(\gamma^{\mu} \partial_{\mu}+\gamma^{5} \partial_{5}\right)-\phi\left(x_{5}\right)\right]\left(\begin{array}{l}\psi \\ \bar{\chi}\end{array}\right)$

where $\gamma^{5}=i$ on $\psi$ and $-i$ on $\bar{\chi}$. A chiral zero mode is localized about the point $x_{5}=x_{5,0}$ where a background scalar field configuration $\phi\left(x_{5}\right)$ equals zero, its wave function being

$$
\psi(y) \propto e^{-\int_{x_{5,0}} d x_{5}^{\prime} \phi\left(x_{5}^{\prime}\right)} .
$$

When the background configuration is approximated by a linear function $\phi\left(x_{5}\right) \approx F\left(x_{5}-x_{5,0}\right)$, then the zero-mode wave function is approximately Gaussian:

$$
\psi(y) \approx e^{-F\left(x_{5}-x_{5,0}\right)^{2} / 2} .
$$

Thus the Gaussian profile follows from the zero-mode equations of motion rather easily. ${ }^{28}$

\footnotetext{
${ }^{27}$ The U(3)-invariant Harr measure for mixing angles follows in basis-independent landscapes [12] when $\lambda^{u}$ and $\lambda^{d}\left(\lambda^{e}, \lambda^{\nu}\right.$, and $\left.c_{\alpha \beta}\right)$ are independent. In landscapes derived from overlap integration, however, there are correlations between the relevant matrices, and hence the mixing angles do not follow the Harr measure.

${ }^{28} \mathrm{On}$ a domain-wall background, the zero-mode wave function is Gaussian in the middle of the domain wall, where $\phi\left(x_{5}\right)$ is approximately linear, and decreases exponentially outside the wall, where $\phi\left(x_{5}\right)$ is nearly constant.
}

However, when the $x_{5}$ direction is compactified into $S^{1}$, the scalar field $\phi\left(x_{5}\right)$ must be periodic. If $\phi\left(x_{5}\right)$ hits zero with a positive slope $k$ times along $S^{1}$, it does with a negative slope $k$ times as well. Thus, when there are $k$ chiral left-handed zero modes in the low-energy effective theory, there must be $k$ chiral right-handed zero modes as well, and the net chirality is zero. Furthermore, the background field configuration $\phi\left(x_{5}\right)$ should satisfy its equation of motion, but kink-anti-kink configurations are in general not stable.

\section{Domain-wall fermion from a $D=6 U(1)$ gauge theory}

These problems are addressed if $\phi\left(x_{5}\right)$ is not a scalar field. Consider, for example, a U(1) gauge theory on a sixdimensional spacetime with a charged fermion:

$$
\mathcal{L}_{6}=\bar{\Psi}_{i} \Gamma^{M} D_{M} \Psi, \quad D_{M}=\partial_{M}-i A_{M},
$$

where the gamma matrices are chosen to be

$$
\Gamma^{M=0,1,2,3}=\gamma^{\mu} \otimes \mathbf{1}, \quad \Gamma^{M=5,6}=\gamma^{5} \otimes \tau^{1,2} .
$$

When the gamma matrices are chosen as above, the $\mathrm{SO}(5,1)$ spinor $\Psi$ consists of four four-dimensional Weyl spinors, $\Psi=\left(\psi_{\uparrow}, \bar{\chi}_{\uparrow}, \psi_{\downarrow}, \bar{\chi}_{\downarrow}\right)^{T}$. In a basis where $\Gamma^{012356}=$ $1 \otimes \tau^{3}$ and

$$
\Gamma^{\mu}=\gamma^{\mu} \otimes \tau^{1}, \quad \Gamma^{5}=\gamma^{5} \otimes \tau^{1}, \quad \Gamma^{6}=1 \otimes i \tau^{2},
$$

the six-dimensional Dirac equation is given by

$$
\begin{aligned}
& \left(\begin{array}{cc}
i \bar{D} & \sigma \cdot \partial \\
\bar{\sigma} \cdot \partial & -i D
\end{array}\right)\left(\begin{array}{l}
\psi_{\uparrow} \\
\bar{\chi}_{\downarrow}
\end{array}\right)=0, \\
& \left(\begin{array}{cc}
i D & \sigma \cdot \partial \\
\bar{\sigma} \cdot \partial & -i \bar{D}
\end{array}\right)\left(\begin{array}{l}
\psi_{\downarrow} \\
\bar{\chi}_{\uparrow}
\end{array}\right)=0,
\end{aligned}
$$

where $\quad D=\left(\partial_{5}-i A_{5}\right)-i\left(\partial_{6}-i A_{6}\right) \quad$ and $\quad \bar{D}=$ $\left(\partial_{5}-i A_{5}\right)+i\left(\partial_{6}-i A_{6}\right)$. Let us focus on the Weyl spinor $\left(\psi_{\uparrow}, \bar{\chi}_{\downarrow}\right)$. When $\partial_{6}$ is ignored, this spinor's Dirac equation is the same as the five-dimensional equation above - that is $A_{6}$ enters the equation just as $\phi\left(x_{5}\right)$ does in (111). Thus $A_{6}$ acts like a mass parameter for a five-dimensional fermion. Chiral fermions are localized where $A_{6}$ "vanishes," just like with the domain-wall fermion. Below we make more precise what we mean by "vanishing" $A_{6}$.

Let us consider a U(1) gauge theory compactified on $T^{2}$, with coordinates $x_{5}$ and $x_{6}$ having periods $L_{5}$ and $L_{6}$. Suppose that $A_{6}$ is linear in $x_{5}$ and $A_{5}$ is constant:

$$
A_{5}=\xi_{5}, \quad A_{6}=F x_{5}+\xi_{6} .
$$

The field strength $F_{56}=F$ is constant, and satisfies an equation of motion $\partial_{m} F^{m n}=0$, where $m, n=5,6$. There is no issue of kink-anti-kink instability (however, see the 
discussion at the end of this section, where another instability is discussed). Since $A_{6}$ is part of a gauge field, it must be periodic in $x_{5} \rightarrow x_{5}+L_{5}$ only up to a gauge transformation. Since a gauge transformation $\varphi\left(x_{6}\right)=$ $e^{2 \pi i x_{6} / L_{6}}$ shifts $A_{6}$ by $2 \pi / L_{6}, A_{6}\left(x_{5}\right)$ and $A_{6}\left(x_{5}+L_{5}\right)$ can differ by an integral multiple of $2 \pi / L_{6}$. Therefore $F L_{5}$ (and the field strength $F$ ) have to be quantized:

$$
F L_{5}=\frac{2 \pi k}{L_{6}}, \quad F=\frac{2 \pi k}{L_{5} L_{6}}, \quad k \in \mathbb{Z} .
$$

It follows from the index theorem that there are

$$
k=\int_{T^{2}} \frac{F}{2 \pi}
$$

chiral fermion zero modes. Because of the linear configuration of the effective mass term $A_{6}$, zero modes correspond only to left-handed spinors (if $k>0$ ), and net chirality is obtained in the low-energy effective theory.

\section{Fermion zero-mode wave functions: I}

It is easy to find one of the $k$ zero-mode wave functions referred to above:

$$
\psi_{\uparrow}\left(x_{5}\right) \approx e^{i \xi_{5} x_{5}} e^{-(F / 2)\left(x_{5}+\left(\xi_{6} / F\right)\right)^{2}}, \quad \bar{\chi}_{\downarrow}\left(x_{5}\right)=0,
$$

just like a domain-wall fermion. This zero mode is localized where the effective mass parameter $A_{6}=F x_{5}+\xi_{6}$ is zero; $x_{5,0}=-\xi_{6} / F$. Since a gauge transformation $\varphi\left(x_{6}\right)=e^{2 \pi i k x_{6} / L_{6}}$ shifts $A_{6}$ by $2 \pi k / L_{6}$ and shifts $-\xi_{6} / F$ by $-L_{5}$, the center coordinate $x_{5,0}$ of the Gaussian profile can always be chosen within the interval $\left[0, L_{5}\right]$. To obtain the other $(k-1)$ zero modes, note that a Kaluza-Klein momentum in the $x_{6}$ direction effectively shifts the Wilson line $\xi_{6}$ :

$$
\begin{aligned}
& \left(\partial_{6}-i \xi_{6}\right) e^{2 \pi i p\left(x_{6} / L_{6}\right)} \psi\left(x_{5}, x_{6}\right) \\
& \quad=e^{2 \pi i p\left(x_{6} / L_{6}\right)}\left[\partial_{6}-i\left(\xi_{6}-\frac{2 \pi p}{L_{6}}\right)\right] \psi\left(x_{5}, x_{6}\right) .
\end{aligned}
$$

Hence the remaining zero modes are

$$
\begin{aligned}
& \psi_{\uparrow}\left(x_{5}, x_{6}\right) \approx e^{i \xi_{5} x_{5}} e^{2 \pi i p\left(x_{6} / L_{6}\right)} e^{-(F / 2)\left(x_{5}+\left(\xi_{6} / F\right)-(p / k) L_{5}\right)^{2}}, \\
& \bar{\chi}_{\downarrow}\left(x_{5}, x_{6}\right)=0,
\end{aligned}
$$

for $p \in \mathbb{Z}(\bmod k)$. The center coordinates of these $k$ Gaussian zero modes are at

$$
x_{5}=-\frac{\xi_{6}}{F}+\frac{p}{k} L_{5} \quad \text { for } p \in \mathbb{Z} / k \mathbb{Z} \text {. }
$$

The width of the Gaussian profile is

$$
d=\frac{1}{\sqrt{F}}=\sqrt{\frac{L_{5} L_{6}}{2 \pi k}} .
$$

It has been assumed that the width of Gaussian wave functions $d$ can be parametrically smaller than the size of the extra dimension in the $x_{5}$ direction. This is equivalent to

$$
\frac{d}{L_{5}}=\frac{1}{\sqrt{2 \pi k u}} \ll 1,
$$

where $u \equiv L_{5} / L_{6}$ parametrizes the shape of $T^{2}$. In the analysis of Sec. III (the Gaussian landscape on $S^{1}$ ), we found $d / L_{5} \approx 0.08$ was sufficient to reproduce the hierarchy of the quark sector. This translates to $u \approx 8$ when $k=3$.

\section{Fermion zero-mode wave functions: II}

In fact the fermion zero-mode wave functions on $T^{2}$ are known exactly; the approximate form (123) is valid only when $\partial_{6}$ can be ignored, i.e. when $L_{6}$ is so small that only one Kaluza-Klein momentum is relevant at a time. Let us take a detour here to see the form of the zero-mode wave functions when $L_{5} \gg L_{6}$ is not satisfied. First, we note that the fermion obeys a twisted periodicity condition,

$$
\begin{aligned}
& \psi_{\uparrow}\left(x_{5}+L_{5}, x_{6}\right)=e^{2 \pi i k\left(x_{6} / L_{6}\right)} \psi_{\uparrow}\left(x_{5}, x_{6}\right), \\
& \psi_{\uparrow}\left(x_{5}, x_{6}+L_{6}\right)=\psi_{\uparrow}\left(x_{5}, x_{6}\right),
\end{aligned}
$$

just like,

$$
\begin{aligned}
& \partial_{m}-i A_{m}\left(x_{5}+L_{5}, x_{6}\right) \\
& =e^{2 \pi i k\left(x_{6} / L_{6}\right)}\left[\partial_{m}-i A_{m}\left(x_{5}, x_{6}\right)\right] e^{-2 \pi i k\left(x_{6} / L_{6}\right)}, \\
& \partial_{m}-i A_{m}\left(x_{5}, x_{6}+L_{6}\right)=\partial_{m}-i A_{m}\left(x_{5}, x_{6}\right) .
\end{aligned}
$$

The wave functions of the $k$ chiral zero modes, which satisfy $i \bar{D} \psi_{\uparrow}=0$, are given by [21]

$$
\begin{aligned}
\psi_{\uparrow}\left(x_{5}, x_{6}\right)^{p}= & C \sum_{m \in \mathbb{Z}} e^{-2 \pi i(m / k) p} e^{i \xi_{5} x_{5}} \\
& \times e^{i F\left(x_{5}+\left(\xi_{6} / F\right)\right)\left(x_{6}+(m / k) L_{6}-\left(\xi_{5} / F\right)\right)} \\
& \times e^{-(F / 2)\left(x_{6}+(m / k) L_{6}-\left(\xi_{5} / F\right)\right)^{2}}
\end{aligned}
$$

$$
=C e^{i \xi_{5} x_{5}} e^{-(F / 2)\left(\tilde{x}_{5}\right)^{2}} e^{(F / 2)\left(\tilde{x}_{5}+i \tilde{x}_{6}\right)^{2}} \vartheta_{0 ; 0}\left(\tilde{v}-\frac{p}{k} ; \tau\right),
$$

for $p \in \mathbb{Z} / k \mathbb{Z}=\{0,1,2, \cdots,(k-1)\}(\bmod k)$. Here we have defined 


$$
\begin{gathered}
\tilde{x}_{5} \equiv x_{5}+\frac{\xi_{6}}{F}, \quad \tilde{x}_{6} \equiv x_{6}-\frac{\xi_{5}}{F}, \quad \tilde{v} \equiv \frac{\tilde{x}_{5}+i \tilde{x}_{6}}{L_{5}}, \\
\tau \equiv \frac{\tau_{0}}{k} ; \quad \tau_{0} \equiv i \frac{L_{6}}{L_{5}} \equiv \frac{i}{u} .
\end{gathered}
$$

In addition, $\vartheta_{0 ; 0}$ is a theta function defined by

$$
\vartheta_{0 ; 0}(v ; \tau)=\sum_{m \in \mathbb{Z}} e^{\pi i \tau m^{2}+2 \pi i m v} .
$$

The wave functions (131) form an orthonormal basis when the normalization constant is

$$
C=\left(\frac{2}{k u}\right)^{1 / 4} \frac{1}{\sqrt{M_{*}^{2} L_{5} L_{6}}} .
$$

If $L_{6} \gg L_{5}$ and $L_{6} \gg 1 / \sqrt{F} \approx \sqrt{L_{5} L_{6}}$, then the zero mode wave function (130) receives dominant contributions only from the $k$ consecutive values of $m$ for which $x_{6,0}=$ $\xi_{5} / F-(m / k) L_{6}$ is between 0 and $L_{6}$. For other values of $m$ the last factor is suppressed by a power of $e^{-(\pi / 2)\left(L_{6} / L_{5}\right)} \ll 1$ when $x_{6} \in\left[0, L_{6}\right]$. Thus the wave functions are linear combinations of Gaussian profiles localized in the $x_{6}$ direction, centered at $x_{6,0}=\xi_{5} / F-(\mathrm{m} / \mathrm{k}) L_{6}$.

If $L_{5} \gg L_{6}$, then the expression given in (130) is not useful to obtain an intuitive picture. Using a modular transformation of the theta function,

$$
\vartheta_{0 ; 0}(v ; \tau)=i \sqrt{\frac{i}{\tau}} e^{-(\pi i / \tau) v^{2}} \vartheta_{0 ; 0}\left(\frac{v}{\tau} ;-\frac{1}{\tau}\right),
$$

(131) can be rewritten

$$
\begin{aligned}
& \psi\left(x_{5}, x_{6}\right)^{p}=C e^{i \xi_{5} x_{5}} e^{-(F / 2)\left(\tilde{x}_{5}\right)^{2}} e^{(F / 2)\left(\tilde{x}_{5}+i \tilde{x}_{6}\right)^{2}} \\
& \times i \sqrt{k u} e^{-(F / 2)\left(\tilde{x}_{5}+i \tilde{x}_{6}-(p / k) L_{5}\right)^{2}} \Theta_{0 ; 0}\left(\boldsymbol{v}^{\prime} ; \tau^{\prime}\right) \\
& =C i \sqrt{k u} e^{i \xi_{5} x_{5}} e^{2 \pi i p\left(\tilde{x}_{6} / L_{6}\right)} e^{-(F / 2)\left(\tilde{x}_{5}-(p / k) L_{5}\right)^{2}} \vartheta_{0 ; 0}\left(\boldsymbol{v}^{\prime} ; \tau^{\prime}\right),
\end{aligned}
$$

where

$$
v^{\prime}=\frac{k L_{5}}{i L_{6}}\left(\frac{\tilde{x}_{5}+i \tilde{x}_{6}}{L_{5}}-\frac{p}{k}\right), \quad \tau^{\prime} \equiv-\frac{1}{\tau}=-\frac{k}{\tau_{0}}=i k u .
$$

When $u \gg 1$, all but one term in the series expansion of the theta function (133) are suppressed by powers of $e^{-\pi k u} \ll$ 1 , while the leading order term is a Gaussian wave function localized at $x_{5,0}=-\xi_{6} / F+(p / k) L_{5}$. This is the solution we obtained in (123).

Although we have chosen a particular gauge (118), the localization pattern of fermion zero modes does not depend on the choice of gauge: the $\mathrm{U}(1)$ gauge transformation on fermion fields is not more than a phase multiplication. Although we chose $A_{6}$ to be $x_{5}$ dependent while $A_{5}$ is not $x_{6}$ dependent, the fermion zero modes exhibit a localization in $x_{5}$ when $L_{6} \ll L_{5}$, and a localization in $x_{6}$ when $L_{5} \ll L_{6}$. Thus the width of the Gaussian profiles is given by (125) in both cases, and is smaller than $L_{5}$ and $L_{6}$, respectively. As expected, despite the $x_{5}-x_{6}$ asymmetric gauge choice, the localization pattern is not $x_{5}-x_{6}$ asymmetric. The modular transformation property of theta function guarantees this.

Note that when $L_{5}$ and $L_{6}$ are comparable, the zeromode wave functions are neither Gaussian nor localized. For more about zero-mode wave functions on tori, see for example [22].

\section{Zero modes of various fields in different representations}

At the beginning of this section, we advertised the idea that all of the fermions, the Higgs boson and the gauge bosons are unified into a super Yang-Mills multiplet of a gauge group $G$ that contains the gauge group of the standard model or some unified theory. Let us describe this idea a little more explicitly, using the language of $T^{2}$ compactification with a $\mathrm{U}(1)$ gauge-field background. Consider a $G=\mathrm{U}(6)$ super Yang-Mills theory (a parallel description applies to Pati-Salam unified theories with $G=\mathrm{SU}(8)$ and $\left.H=\mathrm{SU}(4)_{C} \times \mathrm{SU}(2)_{L} \times \mathrm{SU}(2)_{R}\right)$. Among the generators of the U(6) symmetry, U(1) field strengths on $T^{2}$ are introduced along

$$
\begin{gathered}
\mathbf{t}_{3}=\operatorname{diag}(1,1,1,0,0,0), \quad \mathbf{t}_{2}=\operatorname{diag}(0,0,0,1,1,0), \\
\mathbf{t}_{1}=\operatorname{diag}(0,0,0,0,0,1) .
\end{gathered}
$$

$\mathrm{SU}(3) \times \mathrm{SU}(2)$ and a couple of $\mathrm{U}(1)$ factors commute with these field strengths, and these are identified with the standard model gauge group. Gauginos in the $3 \times 2$ block and in the $1 \times 3$ block are in the $(\mathbf{3}, \mathbf{2})$ and $(\overline{\mathbf{3}}, \mathbf{1})$ representations of $\mathrm{SU}(3)_{C} \times \mathrm{SU}(2)_{L}$, just like the quark doublets and the anti-p-quarks. Thus, the corresponding zero modes of the gaugino can be identified with these standard model fields. Meanwhile, the Higgs boson may arise from $A_{m}$ in the $2 \times 1$ block. Then the gauge interaction of the gauginos in (110) gives rise to the up-type Yukawa coupling: following the commutation relations of U(6) (c.f. [23]) we have,

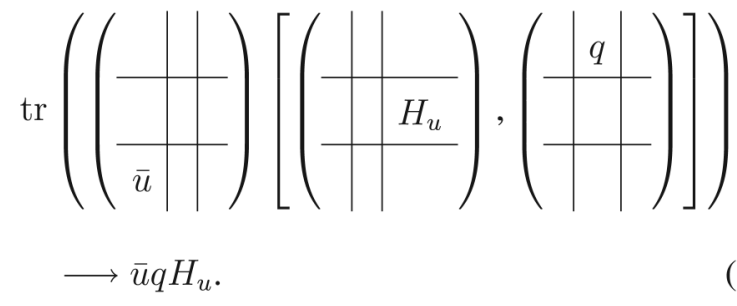

(140) 
The Yukawa couplings are then calculated by overlap integrals of the zero modes.

The $\mathrm{U}(1)$ flux in $\mathrm{U}(6)$ is given by $\mathbf{t}_{3} F^{(3)}+\mathbf{t}_{2} F^{(2)}+$ $\mathbf{t}_{1} F^{(1)}$, with each of $F^{(a)}=\partial_{5} A_{6}^{(a)}(a=1,2,3)$ given by $A_{6}^{(a)}=\left(2 \pi k^{(a)} / L_{5} L_{6}\right) x_{5}+\xi_{6}^{(a)}$, as in (118) and (119). One has to set the field strengths $F^{(3)}, F^{(2)}$, and $F^{(1)}$ so that there are three quark doublets and three anti-up-quarks in the low-energy spectrum. This means that

$$
\begin{aligned}
& \int_{T^{2}} \frac{F^{(3)}}{2 \pi}-\frac{F^{(2)}}{2 \pi}=k^{(3)}-k^{(2)}=3, \\
& \int_{T^{2}} \frac{F^{(1)}}{2 \pi}-\frac{F^{(3)}}{2 \pi}=k^{(1)}-k^{(3)}=3 .
\end{aligned}
$$

The three Gaussian zero-mode wave functions of quark doublets and anti-up-type quarks are localized at

$$
\begin{aligned}
x_{5}^{q_{j}}= & \left(-\frac{\left(\xi_{6}^{(3)}-\xi_{6}^{(2)}\right) L_{6}}{2 \pi\left(k^{(3)}-k^{(2)}\right)}+\frac{j}{k^{(3)}-k^{(2)}}\right) L_{5}, \\
j & \in \mathbb{Z} \bmod \left(k^{(3)}-k^{(2)}\right), \\
x_{5}^{u_{i}^{c}}= & \left(-\frac{\left(\xi_{6}^{(1)}-\xi_{6}^{(3)}\right) L_{6}}{2 \pi\left(k^{(1)}-k^{(3)}\right)}+\frac{i}{k^{(1)}-k^{(3)}}\right) L_{5}, \\
& i \in \mathbb{Z} \bmod \left(k^{(1)}-k^{(3)}\right) .
\end{aligned}
$$

This is an illustration of the picture described in the introduction to this section. We started from U(6) super Yang-Mills theory on $T^{2}$ and broke the $\mathrm{U}(6)$ symmetry by turning on a gauge-field background, so that a $\mathrm{SU}(3)_{C} \times \mathrm{SU}(2)_{L}$ gauge symmetry is left unbroken along with some U(1) factors. Furthermore, the gauge-field background generated chirality in the low-energy spectrum and determined the shape of the zero-mode wave functions (which are Gaussian when $L_{5} \gg L_{6}$ or $L_{6} \gg L_{5}$ ). Wilson lines $\xi_{6}^{(a)}(a=1,2,3)$ are chosen arbitrarily, yet the field strength satisfies the equation of motion. Thus, these constants determine the zero-mode wave functions, and hence the observables that arise from the Yukawa matrices. If the Wilson lines are scanned, the peaks of the Gaussian zero modes are also scanned, and hence the Yukawa couplings are scanned. Since the zero modes of quark doublets and anti-up-type quarks depend differently on the Wilson lines - see (142) and (143) - the peak positions of the wave functions of different representations are scanned independently from one another.

\section{Problems}

The $T^{2}$ compactification of super Yang-Mills theory reproduces certain aspects of the Gaussian landscape on $S^{1}$, but it also has serious problems. The easiest problem to identify involves the sum rule on the chirality. Because of the simple relation $\left(F^{(3)}-F^{(2)}\right)+\left(F^{(2)}-F^{(1)}\right)+\left(F^{(1)}-\right.$ $\left.F^{(3)}\right)=0$, we have

$$
\# \tilde{h}_{u}=-(\# q+\# \bar{u})=k^{(2)}-k^{(1)}=-6,
$$

implying that the low-energy spectrum contains $n \geq 0$ uptype Higgsinos and $6+n$ fermions in a representation conjugate to that of the up-type Higgsinos. One encounters a similar sum rule in any compactification on a smooth two-dimensional manifold with $\mathrm{U}(1)$ field strengths; thus this is too simple a framework to provide both a realistic spectrum and Yukawa interactions.

A more serious problem is that the constant $\mathrm{U}(1) \times$ $\mathrm{U}(1) \times \mathrm{U}(1)$ gauge-field configuration in $\mathrm{U}(6)$ is not stable. Although this configuration satisfies the equations of motion, this only means that it is a stationary point of the action. In fact, the equations of motion of the gauge field in the $3 \times 2$ and $1 \times 3$ blocks are given by

$$
\begin{aligned}
& {\left[\Delta_{56}+\left(F^{(3)}-F^{(2)}\right)_{56}\right]\left(A_{5}+i A_{6}\right)=0,} \\
& {\left[\Delta_{56}+\left(F^{(1)}-F^{(3)}\right)_{56}\right]\left(A_{5}+i A_{6}\right)=0 .}
\end{aligned}
$$

Here $\Delta_{56}$ is the Laplacian on the $x_{5}-x_{6}$ plane. The positive $\left(F^{(3)}-F^{(2)}\right)_{56}$ and $\left(F^{(1)}-F^{(3)}\right)_{56}$ required for the proper quark chirality implies that the low-energy complex scalar fields coming from $A_{5}+i A_{6}$ have negative mass squared. Therefore the constant $\mathrm{U}(1)$ gauge-field configuration is not stable on $T^{2}$. It is stable only when $F_{56}^{(3)}=F_{56}^{(2)}=F_{56}^{(1)}$, which yields no net chirality.

\section{Complex-valued wave functions}

In Sec. VII B we move on to discuss more complicated compactifications of supersymmetric Yang-Mills theory in search of frameworks for constructing Gaussian landscapes that avoid the above problems. For the remainder of this section, however, we digress to explore how $C P$-violating phases can be introduced into these landscapes. We look at the simplest geometry imaginable-compactification on $T^{2}$-considering that the essence of obtaining $C P$-violating phases will not be lost in more complicated frameworks.

Real-valued Gaussian wave functions never yield complex phases in the Yukawa matrices or $C P$-violating phases in the quark and lepton mixing matrices. Although the zero-mode wave functions have phase factors $e^{i \xi_{5} x_{5}} e^{2 \pi i p\left(x_{6} / L_{6}\right)}$, these do not end up as complex phases in the Yukawa matrices. The first factor $e^{i \xi_{5} x_{5}}$ cancels in the integrand of the overlap integration. For example, consider the $\mathrm{U}(6) \rightarrow \mathrm{SU}(3)_{C} \times \mathrm{SU}(2)_{L} \times \mathrm{U}(1)_{Y} \quad$ symmetrybreaking model. The up-type Yukawa operator is neutral under any one of $\mathrm{U}(1)^{(a)}(a=1,2,3)$ symmetries, and this ensures that the $e^{i \xi_{5}^{(3)} x_{5}}$ phase factor in the quark-doublet wave functions is canceled by the factor $e^{-i \xi_{5}^{(3)} x_{5}}$ in the antiup-quark wave functions. As for the second phase factor $e^{2 \pi i p\left(x_{6} / L_{6}\right)}$, recall that the overlap integration is carried out on both the $x_{5}$ and $x_{6}$ coordinates. This phase factor is a 
plane wave associated with Kaluza-Klein momenta, and these momenta are conserved in $T^{2}$ compactifications. Therefore, in any matrix element given by overlap integration on $T^{2}$, these plane wave phase factors cannot yield complex phases. $^{29}$

However, there is a simple way to obtain complex phases in Yukawa matrices without considering more complicated geometries. So far we have assumed $T^{2}$ is rectangular; that is the two independent periods,

$$
\left(x_{5}, x_{6}\right) \sim\left(x_{5}+L_{5}, x_{6}\right), \quad\left(x_{5}, x_{6}\right) \sim\left(x_{5}, x_{6}+L_{6}\right),
$$

are rectangular. In other words, using the complex coordinate $v \equiv\left(x_{5}+i x_{6}\right) / L_{5}$ we have,

$$
v \sim v+1, \quad v \sim v+\tau_{0},
$$

with a pure imaginary $\tau_{0}$ given by (132). When $\tau_{0}$ is not pure imaginary (while keeping the metric $d s^{2}=d x_{5}^{2}+$ $d x_{6}^{2}$ ), zero-mode wave functions have more complicated complex phases, as we see explicitly below.

We now use the gauge-field configuration,

$$
\begin{aligned}
& A_{5}=\xi_{5}, \\
& A_{6}=F\left(x_{5}-\frac{\tau_{1}}{\tau_{2}} x_{6}\right)+\xi_{6}+\frac{\tau_{1}}{\tau_{2}} \xi_{5}=F\left(\tilde{x}_{5}-\frac{\tau_{1}}{\tau_{2}} \tilde{x}_{6}\right),
\end{aligned}
$$

where $\tau_{1}$ and $\tau_{2}$ are the real and imaginary parts of $\tau=$ $\tau_{0} / k$, and

$$
F=\frac{2 \pi k}{L_{5}^{2} \operatorname{Im}\left(\tau_{0}\right)}=\frac{2 \pi}{L_{5}^{2} \tau_{2}} .
$$

The fermion fields satisfy the twisted periodicity conditions in (127) for the periods in (148). The zero-mode wave functions (131) are modified and become

$$
\begin{aligned}
\psi\left(x_{5}, x_{6}\right)^{p} \propto & \sum_{m \in \mathbb{Z}} e^{-2 \pi i(m / k) p} e^{i \xi_{5} x_{5}} \\
& \times e^{i F\left(\tilde{x}_{5}-\left(\tau_{1} / \tau_{2}\right) \tilde{x}_{6}\right)\left(\tilde{x}_{6}+m \tau_{2} L_{5}\right)} e^{\left(\pi i \tau / L_{5}^{2} \tau_{2}^{2}\right)\left(\tilde{x}_{6}+m \tau_{2} L_{5}\right)^{2}}
\end{aligned}
$$

\footnotetext{
${ }^{29}$ Kaluza-Klein momenta conservation leads to an approximate texture in the Yukawa matrices. When only the leading term in the series expansion of the theta function is kept for each of $q, \bar{u}$, and $h$, a given quark doublet has a nonvanishing Yukawa matrix element with only one anti-up-type quark. All of the other matrix elements are suppressed by at least $e^{-\pi u}$, which arises after subleading terms in the series expansion of the theta function are included. On the other hand, when compactifying on generic manifolds there is not necessarily any Kaluza-Klein massless U(1) gauge field and its corresponding conserved Kaluza-Klein momenta. This is why we consider that the Yukawa texture from Kaluza-Klein momenta conservation is an artifact of torus compactification, and have ignored it in the Gaussian landscapes of earlier sections.
}$$
=e^{i \xi_{5} x_{5}} e^{-(\pi i / \tau)(\tilde{v} \bar{\tau}-\bar{v} \tau / \tau-\bar{\tau})^{2}} e^{(\pi i / \tau) \tilde{v}^{2}} \vartheta_{0 ; 0}\left(\tilde{v}-\frac{p}{k} ; \tau\right),
$$

where $\overline{\tilde{v}}$ and $\bar{\tau}$ are the complex conjugates of $\tilde{v}$ and $\tau$, respectively. To see the behavior of these wave functions when $\tau_{2}=\operatorname{Im}\left(\tau_{0}\right)$ is small (roughly equivalent to $u \gg 1$ ), we use (135). Dropping the subleading terms in the series expansion of the theta function and maintaining only the $\mathcal{O}\left(\tau_{2}^{\prime}\right)$ and $\mathcal{O}(1)$ terms in the exponent, we have

$$
\begin{aligned}
\psi^{p}\left(x_{5}, x_{6}\right) \approx & e^{i \xi_{5} x_{5}} e^{\pi i \tau^{\prime}\left(\left(\tilde{x}_{5} / L_{5}\right)-(p / k)\right)^{2}} e^{2 \pi \tau^{\prime}(p / k)\left(\tilde{x}_{6} / L_{5}\right)} \\
& \times e^{-2 \pi \tau_{1}^{\prime}\left(\tilde{x}_{5} \tilde{x}_{6} / L_{5}^{2}\right)} .
\end{aligned}
$$

The factors $e^{i \xi_{5} x_{5}}$ and $e^{2 \pi i \tau_{2}^{\prime}(p / k)\left(\tilde{x}_{6} / L_{5}\right)}$ may cancel in overlap integration. The second factor, ${ }^{30}$

$$
e^{-\pi \tau_{2}^{\prime}\left(1-i \tau_{1}^{\prime} / \tau_{2}^{\prime}\right)\left(\tilde{x}_{5} / L_{5}-p / k\right)^{2}} \sim e^{-(1+r i)\left(\left(\tilde{x}_{5}-(p / k) L_{5}\right)^{2} / 2 d^{2}\right)},
$$

has a complex phase when $r \equiv-\tau_{1}^{\prime} / \tau_{2}^{\prime} \neq 0$. This phase will not cancel with overlap integration on $T^{2}$, which is why we used this second factor in the zero-mode wave functions in the Gaussian landscape of Sec. VID.

\section{B. Doubling $T^{2}-T^{2} \times T^{2}$ compactification of field theory on eight dimensions}

We have seen that the zero-mode wave functions are localized and Gaussian in a $T^{2}$ compactification with a constant U(1) gauge-field background, when $L_{5} \gg L_{6}$ or vice versa. Thus, when the $T^{2}$ is doubled and $T^{4}=T^{2} \times$ $T^{2}$ compactification with a constant $\mathrm{U}(1)$ background is considered, the zero-mode wave functions are localized and Gaussian in two of the four directions, when $T^{4}$ is short in the other two directions. As we will see in this section, in $T^{4}$ compactification the net chirality of various representations is no longer subject to a linear sum rule, and the instability problem in the $T^{2}$ compactification can be avoided when the gauge-field configuration satisfies an anti-self-dual condition. Note that Secs. VII B and VIIC discuss supersymmetric Yang-Mills theory on eight dimensions as a possible origin for Gaussian landscapes, but not because we regard a theory on eight dimensions as a candidate for a fundamental theory. Instead, these sections are intended to provide a step-by-step introduction to ideas that formal theorists are already familiar with, but with minimum technicality. Later, in Secs. VII D and VIIE, we consider a realistic framework for building Gaussian landscapes.

\footnotetext{
${ }^{30}$ Here we have used $2 \pi \tau_{2}^{\prime} \sim\left(L_{5} / d\right)^{2}$; see (126) and (138).
} 


\section{Fermion zero modes in anti-self-dual gauge-field background}

We label the four coordinates of $T^{4} x_{5,6,7,8}$ and the circumferences in these directions are denoted $L_{5,6,7,8}$. We assume that these directions are all orthogonal, i.e. the internal metric is

$$
d s^{2}=\left(d x_{5}\right)^{2}+\left(d x_{6}\right)^{2}+\left(d x_{7}\right)^{2}+\left(d x_{8}\right)^{2} .
$$

On this $T^{2} \times T^{2}$, we introduce a $\mathrm{U}(1)_{2}$ gauge-field configuration embedded in $\mathrm{SU}(2)$ :

$$
\begin{array}{cc}
A_{5}^{(2)}=\xi_{5}^{(2)} \tau^{3}, & A_{6}^{(2)}=\left(\frac{2 \pi k}{L_{5} L_{6}} x_{5}+\xi_{6}^{(2)}\right) \tau^{3}, \\
A_{7}^{(2)}=-\xi_{7}^{(2)} \tau^{3}, & A_{8}^{(2)}=-\left(\frac{2 \pi k^{\prime}}{L_{7} L_{8}} x_{7}+\xi_{8}^{(2)}\right) \tau^{3} .
\end{array}
$$

The field strength is constant and satisfies the equation of motion. Moreover, this configuration is stable when the anti-self-dual condition $F=-* F$ is satisfied; that is when

$$
F_{56}=-F_{78}, \quad \frac{2 \pi k}{L_{5} L_{6}}=\frac{2 \pi k^{\prime}}{L_{7} L_{8}} .
$$

A Weyl fermion in the fundamental representation of the SU(2) has $2 k k^{\prime}$ zero modes on $T^{4}$. To see this, we use the following gamma matrices:

$$
\begin{gathered}
\Gamma^{\mu=0,1,2,3}=\gamma^{\mu} \otimes 1 \otimes 1, \quad \Gamma^{5,6}=\gamma^{5} \otimes \tau^{1,2} \otimes 1, \\
\Gamma^{7,8}=\gamma^{5} \otimes \tau^{3} \otimes \tau^{1,2} .
\end{gathered}
$$

A Weyl fermion on eight dimensions consists of fourdimensional Weyl fermions $\left(\psi_{\uparrow \downarrow}^{a}, \psi_{\downarrow \uparrow}^{a}, \bar{\chi}_{\Uparrow \uparrow}^{a}, \bar{\chi}_{\downarrow \downarrow}^{a}\right)$, where $a$ is a "color" index running $a=1,2$ in the case of a fermion in the fundamental representation of SU(2). Corresponding to the $k k^{\prime}$ different choices of $p \in \mathbb{Z} / k \mathbb{Z}$ and $p^{\prime} \in \mathbb{Z} / k^{\prime} \mathbb{Z}$, the Dirac equation on $T^{4}$ with the gauge-field background (157) has $k k^{\prime}$ zero modes in $\psi^{a=1}$ :

$$
\begin{gathered}
\psi_{\uparrow \downarrow}^{a=1 ; p, p^{\prime}}=\psi_{\uparrow}\left(x_{5}, x_{6} ; k\right)^{p} \psi_{\uparrow}\left(x_{7}, x_{8} ; k^{\prime}\right)^{p^{\prime}}, \\
\psi_{\downarrow \uparrow}^{a=1 ; p, p^{\prime}}=0, \quad \psi^{a=2 ; p, p^{\prime}}=0, \quad \bar{\chi}=0,
\end{gathered}
$$

Likewise, there are also $k k^{\prime}$ zero modes in $\psi^{a=2}$ :

$$
\begin{gathered}
\psi_{\downarrow \uparrow}^{a=2 ; p, p^{\prime}}=\psi_{\uparrow}\left(x_{5}, x_{6} ; k\right)^{p} \psi_{\uparrow}\left(x_{7}, x_{8} ; k^{\prime}\right)^{p^{\prime}}, \\
\psi_{\uparrow \downarrow}^{a=2 ; p, p^{\prime}}=0, \quad \psi^{a=1 ; p, p^{\prime}}=0, \quad \bar{\chi}=0 .
\end{gathered}
$$

When $L_{5} \gg L_{6}$ and $L_{7} \gg L_{8}$, all of these wave functions are approximately Gaussian in the $x_{5}-x_{7}$ plane, since the wave function is a product of Gaussians in the $x_{5}$ and $x_{7}$ directions. Two zero modes, one in $\psi_{\uparrow \downarrow}^{a=1}$ and the other in $\psi_{\downarrow \uparrow}^{a=2}$, are localized at each of

$$
\left(x_{5}, x_{7}\right)=\left(\left(-\frac{\xi_{6} L_{6}}{2 \pi k}+\frac{p}{k}\right) L_{5},\left(-\frac{\xi_{8} L_{8}}{2 \pi k^{\prime}}+\frac{p^{\prime}}{k^{\prime}}\right) L_{7}\right) .
$$

The width of all of these zero modes is $d=1 / \sqrt{\left|F_{56}\right|}$ in the $x_{5}$ direction and $d^{\prime}=1 / \sqrt{\left|F_{78}\right|}$ in the $x_{7}$ direction. The anti-self-dual condition (158) implies that the two widths $d$ and $d^{\prime}$ are equal, as was assumed in the $D=2$ Gaussian landscapes of Sec. V.

\section{Absence of a chirality sum rule}

The two serious problems of $T^{2}$ compactification with a $\mathrm{U}(1)$ gauge-field background derived from instability of the gauge-field configuration and the sum rule satisfied by the chirality of different representations. The anti-self-dual condition (158) addresses the first issue in $T^{4}$ (or in any four-dimensional manifold) compactification, while in what follows we see that there is hope to resolve the second issue in four-fold compactifications as well.

The chirality sum rule on $T^{2}$ derived from the Yukawa interactions being consistent with the gauge symmetry of an underlying group $G$. Therefore, to discuss the existence of such a sum rule ${ }^{31}$ on $T^{4}$, we must consider constraints from the underlying gauge symmetry whose super YangMills interactions give rise to the Yukawa couplings of quarks and leptons. Here we consider $G=E_{6}$ and $H=$ $\mathrm{SU}(5)_{\mathrm{GUT}}$. $E_{6}$ contains a subgroup

$$
E_{6} \supset \mathrm{SU}(2) \times \mathrm{SU}(6) \supset \mathrm{SU}(2) \times \mathrm{U}(1)_{6} \times \mathrm{SU}(5)_{\mathrm{GUT}} .
$$

The $H=\mathrm{SU}(5)_{\mathrm{GUT}}$ symmetry is unbroken when a background gauge field on $T^{4}$ is contained within $\mathrm{SU}(2) \times$ $\mathrm{U}(1)_{6}$. We use the $\mathrm{U}(1)_{2}$ gauge-field configuration $A_{m}^{(2)}$ in (157), embedded in the SU(2) factor, and introduce a $U(1)$ gauge-field background in the $\mathrm{U}(1)_{6}$ factor, given by

$$
A_{6}^{(6)}=\frac{2 \pi k^{\prime \prime}}{L_{5} L_{6}} x_{5}+\xi_{6}^{(6)}, \quad A_{8}^{(6)}=-\left(\frac{2 \pi k^{\prime \prime \prime}}{L_{7} L_{8}} x_{7}+\xi_{8}^{(6)}\right),
$$

with another anti-self-dual condition $2 \pi k^{\prime \prime} / L_{5} L_{6}=$ $2 \pi k^{\prime \prime \prime} / L_{7} L_{8}$.

The irreducible decomposition of the $E_{6}$ Lie algebra,

$$
\mathfrak{e}_{6}-\operatorname{adj} . \rightarrow(\operatorname{adj} ., 1)+(1, \operatorname{adj} .)+\left(\wedge^{3} \mathbf{6}, \mathbf{2}\right),
$$

\footnotetext{
${ }^{31}$ To be precise, chirality on four dimensions is not obtained from a super Yang-Mills theory on eight dimensions compactified on $T^{4}$ with an anti-self-dual gauge-field configuration. This is because the super Yang-Mills multiplet contains a pair of Weyl fermions with opposite chirality. When a zero mode is found in $\psi_{\uparrow \downarrow}^{a=1}\left(\psi_{\downarrow \uparrow}^{p=2}\right)$ in a Weyl fermion $\left(\psi_{\uparrow \downarrow}, \psi_{\downarrow \uparrow}, \bar{\chi}_{\uparrow \uparrow}, \bar{\chi}_{\downarrow \downarrow}\right)$, another Weyl fermion with the opposite chirality, $\left(\psi_{\uparrow \downarrow}, \psi_{\downarrow \uparrow}, \chi_{\uparrow \uparrow}, \chi_{\Downarrow \downarrow}\right)$, also has a zero mode $\bar{\psi}_{\uparrow \downarrow}^{p=1}\left(\psi_{\downarrow \downarrow}^{a=2}\right)$. The chirality we refer to in this section concerns $T^{4}$ compactification of ten to six dimensions, which has nothing to do with the chirality on four dimensions. As we explain later, however, the chirality on six dimensions in $T^{4}$ compactification has nonlinearity, which is shared by chirality on four dimensions in any six-dimensional compactification. This "chirality" is discussed in the context of $T^{4}$ compactification because this is the simplest system with this nonlinearity.
} 
$\rightarrow(\operatorname{adj} ., 1)^{0}+(\mathbf{5}, 1)^{6}+$ H.c. $+(1$, adj. $)+\left(\wedge^{2} \mathbf{5}, \mathbf{2}\right)^{-3}$

+ H.c.,

shows that $\mathfrak{g} / \mathfrak{h}$ contains both $\left(\wedge^{2} \mathbf{5}, \mathbf{2}\right)^{-3}$ and $(\mathbf{5}, \mathbf{1})^{6}$, candidates for the $\mathbf{1 0}=(q, \bar{u}, \bar{e})$ and $H(\mathbf{5})$ multiplets, respectively. The above decomposition refers to the $\mathrm{SU}(6) \times$ $\mathrm{SU}(2)$ subgroup in the first line, and $\mathrm{SU}(5)_{\mathrm{GUT}} \times \mathrm{U}(1)_{6} \times$ $\mathrm{SU}(2)$ in the second line. Gauge interactions of $\mathfrak{e}_{6}$ gauginos may in principle give rise to the four-dimensional up-type Yukawa couplings 32,33

$$
\mathcal{L}_{4}=\psi_{10} \cdot \psi_{10} \cdot \phi_{H(5)} .
$$

As a quick check, note that in the $\mathfrak{e}_{6}$ algebra $\operatorname{tr}\left(\left(\wedge^{2} \mathbf{5}, \mathbf{2}\right)^{-3}\left[(\mathbf{5}, \mathbf{1})^{+6},\left(\wedge^{2} \mathbf{5}, \mathbf{2}\right)^{-3}\right]\right)$ does not vanish. The coefficients (Yukawa matrix elements) are calculated by the overlap integration of the zero-mode wave functions, picking up a pair of $\psi_{\uparrow \downarrow}^{p=1}$ and $\psi_{\downarrow \uparrow}^{p=2}$. If the compactification preserves $\mathcal{N}=1$ supersymmetry, a complex scalar and a chiral fermion in a chiral multiplet have the same wave functions on the internal manifold. Therefore, the wave function of the Higgs boson is Gaussian, whenever the Higgsino wave function is Gaussian.

The number of zero modes is calculated in terms of the flux quanta $k, k^{\prime}, k^{\prime \prime}$, and $k^{\prime \prime \prime}$. Up-type Higgsinos in lowenergy spectrum are zero modes of the Dirac equation involving a $\mathrm{U}(1)$ gauge field $6 A_{m}^{(6)}$. Therefore, the number

\footnotetext{
${ }^{32}$ Let us first consider how gauge indices are contracted. The $\mathrm{SU}(5)_{\mathrm{GUT}}$ indices of the two fermions $\psi_{\mathbf{1 0}}$ are contracted with the $\mathrm{SU}(5)_{\text {GUT }}$ totally antisymmetric tensor, and the contraction is symmetric under the exchange of the two. On the other hand, the internal gauge indices of SU(2) are antisymmetric as the above interaction is neutral under the underlying gauge symmetry $\mathrm{SU}(2) \times \mathrm{U}(1)_{6}$, when the contraction is in the first term of the right-hand side of $\mathbf{2}^{-3} \otimes \mathbf{2}^{-3} \otimes \mathbf{1}^{+6} \simeq \wedge^{2} \mathbf{2} \oplus \cdots$. Generally, gauge indices are contracted antisymmetrically under the exchange of the two zero modes in the $\left(\wedge^{2} \mathbf{5}, 2\right)^{-3}$ representation. This should be the case because the gauge indices are contracted through the structure constant of a Lie algebra in (110). Spinor indices are contracted symmetrically under the exchange of $\psi_{10}$ 's; both the $\operatorname{SO}(3,1)$ contraction of left-handed spinors in four dimensions and the contraction of spinor indices of $\mathrm{SU}(2) \subset \mathrm{SO}(4)$ in the internal space are antisymmetric. Therefore the combined contraction of gauge (antisymmetric) and spinor (symmetric) indices is antisymmetric. This is expected since the gauginos in eight dimensions are Grassmann variables.

${ }^{33}$ The Higgs scalar should, then, originate from the complex scalar of a super Yang-Mills multiplet on eight dimensions. However, because of the $\mathcal{N}=2$ supersymmetry, preserved in $T^{4}$ compactification with an anti-self-dual gauge-field configuration, the existence of massless Higgs boson implies that its $\mathcal{N}=2$ super partner, a vector field in the same representation, should also be massless. This implies that the symmetry is not broken down to $\mathrm{SU}(5)_{\mathrm{GUT}}$. As we have seen, $T^{4}$ compactification with an anti-self-dual configuration has so many problems that it cannot be regarded as a realistic framework for landscapes. Our goal in Sec. VIIB is to introduce field-theory ideas that apply to many compactifications, using the language of $T^{4}$ compactification.
}

of up-type Higgsino zero modes is

$$
\# H_{u}=\left(6 k^{\prime \prime}\right)\left(6 k^{\prime \prime \prime}\right)=36 k^{\prime \prime} k^{\prime \prime \prime} .
$$

Light fermions in the $\mathbf{1 0}$ representation are zero modes of a Dirac equation with gauge field $A^{(2)}-3 A^{(6)}$. The number of zero modes is

$$
\begin{aligned}
\# \mathbf{1 0} & =\left(k-3 k^{\prime \prime}\right)\left(k^{\prime}-3 k^{\prime \prime \prime}\right)+\left(k+3 k^{\prime \prime}\right)\left(k^{\prime}+3 k^{\prime \prime \prime}\right) \\
& =2 k k^{\prime}+18 k^{\prime \prime} k^{\prime \prime \prime},
\end{aligned}
$$

with the first term coming from those in $\psi^{a=1}$ and the second term from those in $\psi^{a=2}$. We have four (discrete) parameters to choose by hand to fit the desired multiplicities of the fields in the two different representations, $H_{u}$ and $\mathbf{1 0 .}$

The discussion so far has not been realistic. The above multiplicities count only the number of hypermultiplets of $\mathcal{N}=2$ supersymmetry in four dimensions. We have also commented in footnote 33 that the Higgs scalar field in the up-type Yukawa couplings should belong to an $\mathcal{N}=2$ vector multiplet. However, it will now be easy to take one more step and triple $T^{2}$ to a $T^{6}$ compactification of a super Yang-Mills theory in ten dimensions. Chirality on four dimensions is obtained in $D=6$ compactifications, and the net chirality of a given representation is cubic in discrete parameters specifying the fluxes on $T^{6}$. No sum rule holds among the chirality of various representations, since they are not linear functions of the flux parameters. As more flux parameters are involved, it is easier to fit the multiplicities of fields in various representations. It is also known that one of the gauge background stability conditions is $F_{\alpha \bar{\beta}} g^{\alpha \bar{\beta}}=0$ (in compactifications preserving $\mathcal{N}=1$ supersymmetry), and the $T^{2}$ compactification has instability because only one term contributes to the lefthand side, with no chance of cancellation. Hence this instability is an artifact of the $D=2$ compactification. $E_{8}$ contains $E_{6}$, and therefore the algebra that led to the up-type Yukawa couplings still works in $E_{8}$. Thus super Yang-Mills theories of ten dimensions compactified on sixdimensional internal manifolds can be a good theoretical framework for building Gaussian landscapes.

\section{Scanning of the center coordinates}

Before closing this section, let us see how the localized wave functions behave as the constant gauge-field background on $T^{4}$ changes. We already know that all the zeromode wave functions are approximately Gaussian if $L_{5} \gg$ $L_{6}$ and $L_{7} \gg L_{8}$. The center coordinates of these wave functions depend on the Wilson lines $\xi_{6}^{(2),(6)}$ and $\xi_{8}^{(2),(6)}$, according to

$$
\left(x_{5}^{H_{u}}, x_{7}^{H_{u}}\right)=\left(\left(\frac{p_{H_{u}}}{6 k^{\prime \prime}}-\frac{\xi_{6}^{(6)} L_{6}}{2 \pi k^{\prime \prime}}\right) L_{5},\left(\frac{p_{H_{u}}^{\prime}}{6 k^{\prime \prime \prime}}-\frac{\xi_{8}^{(8)} L_{8}}{2 \pi k^{\prime \prime \prime}}\right) L_{7}\right),
$$




$$
\begin{aligned}
\left(x_{5}^{\mathbf{1 0}}, x_{7}^{\mathbf{1 0}}\right)_{a=1}= & \left(\left(\frac{p_{\mathbf{1 0}}^{a=1}}{k-3 k^{\prime \prime}}-\frac{\left(\xi_{6}^{(2)}-3 \xi_{6}^{(6)}\right) L_{6}}{2 \pi\left(k-3 k^{\prime \prime}\right)}\right) L_{5},\right. \\
& \left.\left(\frac{p_{\mathbf{1 0}}^{a=1}}{k^{\prime}-3 k^{\prime \prime \prime}}-\frac{\left(\xi_{8}^{(2)}-3 \xi_{8}^{(8)}\right) L_{8}}{2 \pi\left(k^{\prime}-3 k^{\prime \prime \prime}\right)}\right) L_{7}\right), \\
\left(x_{5}^{\mathbf{1 0}}, x_{7}^{\mathbf{1 0}}\right)_{a=2}= & \left(\left(\frac{p_{\mathbf{1 0}}^{a=2}}{k+3 k^{\prime \prime}}-\frac{\left(\xi_{6}^{(2)}+3 \xi_{6}^{(6)}\right) L_{6}}{2 \pi\left(k+3 k^{\prime \prime}\right)}\right) L_{5},\right. \\
& \left.\left(\frac{p_{\mathbf{1 0}}^{\prime a}=2}{k^{\prime}+3 k^{\prime \prime \prime}}-\frac{\left(\xi_{8}^{(2)}+3 \xi_{8}^{(8)}\right) L_{8}}{2 \pi\left(k^{\prime}+3 k^{\prime \prime \prime}\right)}\right) L_{7}\right),
\end{aligned}
$$

where the coordinates in the first line are those of up-type Higgsino(s), while the second and third lines are those of the 10 fermions, with the second and third lines coming from $\psi^{a=1}$ and $\psi^{a=2}$, respectively. Note that the coordinates of the second and third lines show different dependences on the Wilson lines. Thus, as the Wilson lines are scanned, the localized fermions in the $\mathbf{1 0}$ representation of $\mathrm{SU}(5)_{\text {GUT }}$ change their relative positions. We see that the scanning of Wilson lines allows for more than the centerof-mass scanning of Gaussian peaks to zero-mode wave functions in $T^{4}$ compactification. In the Gaussian landscapes we scanned the peak positions of Gaussian wave functions without considering any correlations or constraints among the various peak positions. The above situation is still far from this treatment, but so far we have only scanned the Wilson lines. In Sec. VIIC we see that there are other stable gauge-field configurations, and as more gauge-field configurations are scanned, correlation will be lost among the Gaussian peak positions. This is the rationale behind the absence of correlations in the Gaussian landscapes.

\section{Instanton moduli and random peak-position scanning}

\section{Instanton moduli}

We have now seen an explicit example of a stable gaugefield background on $T^{4}$ parameterized by Wilson lines. In general, stable gauge-field configurations allow for continuous deformations, such as Wilson lines, and the parameters of such deformations are called moduli. As for gauge-field configurations on a four-dimensional manifold, we know that those satisfying the anti-self-dual condition satisfy both the Yang-Mills theory equations of motion and the stability condition. Such gauge-field configurations, called instantons, are known to have numerous moduli parameters; the Wilson lines on $T^{4}$ are just a subset of the instanton moduli parameters. Therefore we now consider what happens when the instanton moduli parameters are scanned. Our primary interest is to explore the moduli space of stable gauge-field configurations on a sixdimensional manifold, as this is relevant to the compactification of the heterotic string theory. However, to warm up we first study instanton moduli on a four-dimensional manifold.

The $E_{6} \rightarrow \mathrm{SU}(5)_{\mathrm{GUT}}$ symmetry-breaking model in Sec. VII B generates the up-type Yukawa couplings. As long as a background configuration of $e_{6}$ gauge field is contained in its $\mathfrak{S H}(2)+\mathfrak{H}(1)_{6}$ subalgebra, the $\mathrm{SU}(5)_{\text {GUT }}$ symmetry remains unbroken. Note that the gauge-field configuration does not have to be pure Abelian over the entire $T^{4}$ as is assumed in (157). The gauge-field configuration $A^{(2)}$ in (157) can be replaced by any one of the SU(2) instanton configurations ${ }^{34}$ on $T^{4}$. The 't Hooft solution is a SU(2) instanton configuration on a flat Euclidean fourdimensional space [24]; it has 5I moduli parameters, where the instanton number $I$ is topological and defined by

$$
\begin{aligned}
I & =-\int \operatorname{ch}_{2, \text { fund. }}(F)=-\int \operatorname{tr}_{\text {fund. }}\left[\frac{1}{2}\left(\frac{F}{2 \pi}\right)^{2}\right] \\
& =-\frac{1}{2 T_{R}} \int \operatorname{ch}_{2, R}(F)
\end{aligned}
$$

here "fund." stands for fundamental representation, $R$ is an arbitrary representation, and " $\mathrm{ch}_{2 ; R}$ " stands for the second Chern character in the representation $R$. The gauge-field configuration $A^{(2)}$ in (157) has $I=2 k k^{\prime}$ instantons. Meanwhile, the 't Hooft solution,

$$
A_{m}^{a}=-\bar{\eta}_{m n}^{a} \partial_{n} \ln \left(1+\sum_{j=1}^{I} \frac{\rho_{j}^{2}}{\left(y-y_{j}\right)^{2}}\right)
$$

describes $I$ isolated instantons, centered at $\left(y_{j}\right)_{m}$ with a size $\rho_{j}$. Here, $a=1,2,3$ label the three generators of $\mathfrak{S H}(2)$ and $\bar{\eta}_{m n}^{a}$ is the eta symbol of 't Hooft. Thus there are indeed $(4+1) I$ moduli parameters. In addition to these five moduli parameters per instanton, there are three more moduli parameters describing how an instanton solution is embedded within the group $\mathrm{SU}(2)$. Thus, there are (roughly) $8 I$ moduli parameters for the SU(2) I-instanton configuration.

On a compact four-dimensional manifold $X$, the number of instanton moduli parameters of a gauge group $G$ is given

\footnotetext{
${ }^{34}$ The number of fermion zero modes is given by the topology of the gauge-field configuration and of the geometry, as in the case of $T^{2}$ compactification. It follows from the index theorem that the number of fermion zero modes in a representation $R$ of a gauge-field background is

$$
-\int_{X} \operatorname{ch}_{R}(F) \hat{A}(T X)=-\int_{X} \operatorname{ch}_{2 ; R}(F)+\frac{\operatorname{dim} R}{24} \int_{X} p_{1}(T X),
$$

where $\hat{A}(T X)$ is the $\hat{A}$ classes of $X$ and $p_{1}(T X)$ is in the first Pontrjagin class. This expression is valid for an arbitrary fourdimensional manifold $X$. The first term on the right-hand side is equal to $2 T_{R} I$, that is it is proportional to the instanton number $I$. As long as the instanton number remains the same, the net chirality does not change for any instanton configuration. See the text for the definitions of $\operatorname{ch}_{2, R}(F)$ and $I$.
} 
by [25]

$$
\begin{aligned}
-2 \int_{X} \operatorname{ch}_{2 ; \text { adj. }}(F) t d(T X)= & 4 T_{G} I-\frac{\operatorname{dim} G}{6} \\
& \times \int_{X} c_{2}(T X)+c_{1}(T X)^{2},
\end{aligned}
$$

where $T_{G}$ denotes the Dynkin index in the adjoint representation (also known as the dual Coxeter number), $t d(T X)$ is the Todd classes, and $\operatorname{dim} G$ is the dimension of $G$. When $G$ is $\mathrm{SU}(N), T_{G}=N$ and $\operatorname{dim} G=N^{2}-1$. Apart from the second term, which is associated with compactness of the four-fold $X$, the first term reproduces the result of $G=\mathrm{SU}(2)-8 I$ moduli parameters. The second term corresponds to an obstruction for lifting isolated $I$ instantons into an anti-self-dual configuration over all of the $X$ (c.f. [26]). The Wilson lines $\xi_{5,6,7,8}^{(2)}$ on the $T^{4}$ compactification of Sec. II B are part of the instanton moduli. However, unlike the Wilson lines of $T^{4}$, which are associated with a nontrivial $\pi_{1}\left(T^{4}\right)$, most of instanton moduli here are not strongly associated with the specific geometry of $T^{4}$. Thus the existence of instanton moduli is very robust.

\section{Wilson lines as instanton moduli parameters}

So far it has not been clear how the Wilson lines on $X=$ $T^{4}$ are related to the instanton moduli parameters, such as $\left(y_{j}\right)_{m}$ and $\rho_{m}$, in the 't Hooft solution (175); in particular, the constant-field configuration does not have a welldefined center. To see explicitly that the Wilson lines actually correspond to instanton center coordinates, ADHM data [27] and the Nahm transformation [28] are useful.

The ADHM formalism [27] allows one to parametrize the moduli space of an instanton configuration on a flat space $\mathbb{R}^{4}$. A set of parameters called ADHM data describes all possible instanton gauge-field configurations and, in turn, the data can be extracted starting from the gauge-field configuration. For simplicity, we consider only the $I$-instanton configurations of a $\mathrm{SU}(N)$ gauge group. Among the data is an $I \times I$ matrix-valued $\hat{A}_{m}$. This part of the data is extracted by

$$
\left(\hat{A}_{m}\right)^{p^{\prime} p}=\left\langle p^{\prime}\left|x_{m}\right| p\right\rangle=\int_{\mathbb{R}^{4}} d^{4} x \psi^{p^{\prime} \dagger} x_{m} \psi^{p},
$$

where $\psi^{p}\left(p, p^{\prime}=1, \cdots, I\right)$ are the zero-mode wave functions of a fermion in the fundamental representation of the $\mathrm{SU}(N)$ gauge group. Since the zero-mode wave functions are determined by the gauge-field configuration used in the Dirac equation on $\mathbb{R}^{4}$, the data $\hat{A}_{m}$ carry some of the information of the gauge-field configuration. It is known that the data corresponding to the 't Hooft solution are

$$
\left(\hat{A}_{m}\right)^{p^{\prime} p}=\left(y_{p}\right)_{m} \delta_{p^{\prime} p} .
$$

Thus the data $\hat{A}_{m}$ provide a way to extract "center coordinates" from the instanton gauge-field configuration. When one considers a family of instanton gauge-field configurations by modifying

$$
A_{m}(x) \rightarrow A_{m}^{\prime}(x)=A_{m}(x)+\xi_{m},
$$

the fermion zero-mode wave functions change according to

$$
\psi(x) \rightarrow \psi^{\prime}(x)=e^{i \xi_{m} x_{m}} \psi(x)
$$

Therefore, the data $\left\langle p^{\prime}\left|x_{m}\right| p\right\rangle$ are also extracted by

$$
\begin{aligned}
\left(\hat{A}_{m}^{\prime}\right)^{p^{\prime} p} & =\left\langle p^{\prime}\left|x_{m}\right| p\right\rangle=\left\langle p^{\prime}\left|-i \partial_{\xi_{m}}\right| p\right\rangle \\
& =-i \int_{\mathbb{R}^{4}} d^{4} x \psi^{\prime p^{\prime} \dagger} \partial_{\xi_{m}} \psi^{\prime p} .
\end{aligned}
$$

The gauge-field configuration (157) is already in the form (179), and the zero-mode wave functions of the fermions in the fundamental representation are also already provided. The corresponding data, now calculated by integration on $T^{4}$, not $\mathbb{R}^{4}$, are ([21] and references therein)

$$
\begin{aligned}
\left(\hat{A}_{5,6,7,8}^{\prime}\right)^{p^{\prime} p} & =\left(-\frac{\xi_{6}^{(2)}}{\left|F_{56}\right|}, 0,-\frac{\xi_{8}^{(2)}}{\left|F_{78}\right|}, 0\right) \delta_{p^{\prime} p} \\
& =\left(-\left(\frac{\xi_{6}^{(2)} L_{6}}{2 \pi k}\right) L_{5}, 0,-\left(\frac{\xi_{8}^{(2)} L_{8}}{2 \pi k^{\prime}}\right) L_{7}, 0\right) \delta_{p^{\prime} p} .
\end{aligned}
$$

Therefore, the Wilson lines $\xi_{6}^{(2)}$ and $\xi_{8}^{(2)}$ can be regarded as center-of-mass modes of the instanton center coordinates in the appropriate limit of the instanton moduli space.

\section{Scanning over instanton moduli space}

If the moduli parameters of the instanton configurations are scanned randomly (according to some measure), the gauge-field configuration is determined for each choice of moduli parameter, as are the zero-mode wave functions. In the $E_{6} \rightarrow \mathrm{SU}(5)_{\mathrm{GUT}}$ symmetry-breaking model of Sec. VII B, fermions in the $\mathbf{1 0}$ representation of $\mathrm{SU}(5)_{\mathrm{GUT}}$ in the effective theory are zero modes of fermions in the $2^{-3}$ representation of an $\mathrm{SU}(2) \times \mathrm{U}(1)_{6}$ instanton background. As the moduli of a SU(2) instanton are scanned, zero-mode wave functions of the fields in $\mathbf{1 0}=$ $(q, \bar{u}, \bar{e})$ vary. The center coordinates of these wave functions will be scanned almost randomly, since the instanton center coordinates can be chosen arbitrarily in (175). The zero modes of a fermion in the $\mathbf{2}^{-3}$ representation are not classified into the zero modes in $\psi^{a=1}$ and those in $\psi^{a=2}$. The instanton configuration is contained in a Cartan $\mathrm{U}(1)_{2}$ subgroup for only limited points in the instanton moduli space; for generic points in the moduli space the SU(2) symmetry is completely broken and the distinction between $a=1$ and $a=2$ is lost. In general each zero mode has nonzero wave functions in both $a=1$ and 
$a=2$. This means that there is no selection rule in the Yukawa couplings; if some zero modes were exclusively in $a=1$ and all others were in $a=2$, then the Yukawa couplings would have involved only a pair of zero modes, one from each group.

The Gaussian landscapes of Secs. III, V, and VI extracted these features. In particular, we applied these features to all of the fields in the standard model. Certainly the Higgs wave function is determined only by a gauge-field background of an Abelian symmetry $\mathrm{U}(1)_{6}$ in the $E_{6} \rightarrow$ $\mathrm{SU}(5)_{\mathrm{GUT}}$ symmetry-breaking model; therefore, the complexity (and variety) of instanton field configurations of non-Abelian symmetries has nothing to do with the scanning of the Higgs field's center coordinate. But this is just an artifact of choosing $G$ to be minimal for the up-type Yukawa couplings. For a larger underlying gauge symmetry $G$, such as $G=E_{8}$, the Higgs boson is regarded as a zero mode of a field in the $\wedge^{2} \mathbf{5}$ representation in $E_{8} \rightarrow$ $\mathrm{SU}(5)_{\mathrm{GUT}}$ symmetry breaking due to a $\mathrm{SU}(5)$ instanton. Gaussian landscapes are based on an expectation that the variety of stable gauge-field configurations is so rich that the random scanning of gauge-field moduli results in (approximately) random and independent scanning of the center coordinates of the zero-mode wave functions.

One will notice here that the three Gaussian zero-mode wave functions in a given representation do not necessarily satisfy the orthonormal condition (109), when the center coordinates are chosen completely randomly. Our approach has been to try to implement the rich scanning of gauge-field moduli in Gaussian landscapes at the cost of giving up basis independence. Since the orthonormal condition is violated especially when two center coordinates coincide, the probability distribution functions of the smaller eigenvalues of a Yukawa matrix in Gaussian landscapes may become unreliable as they approach the largest possible value, $z_{i} \sim 0$ in the notation of this paper. Although the center coordinates of two instantons can coincide, the two zero-mode wave functions associated with them should be properly modified so that they remain orthogonal. This modification is not taken into account in the Gaussian landscape. It is a yet-to-be-tested question in string theory whether the gauge-field moduli are such that the random scanning of center coordinates is a relatively good approximation or not, and if not, how the correct distribution functions of observables would deviate from the predictions of Gaussian landscapes.

\section{Non-Gaussian wave functions}

We here note that zero-mode wave functions are not always Gaussian for arbitrary choice of gauge-field moduli parameters. In fact, when the sizes of instantons $\rho_{j}$ in the 't Hooft solution (175) are much smaller than the typical distance between the instanton centers $\left|y_{k}-y_{l}\right|$, the ' $t$ Hooft solution is a collection of isolated Belavin,
Polyakov, Shvarts, and Tyrupkin instantons. Fermion zero modes are localized around the instanton centers $y \sim$ $y_{j}$, and their wave functions decay in as a power of the distance $\left|y-y_{j}\right|$, not exponentially. Unless the size parameters $\rho_{j}$ are extremely small, however, overlap integration using these nonexponential wave functions tends to be larger than the $10^{-5}-10^{-6}$ required to match the quark and lepton Yukawa couplings of the first generation.

There are also situations where zero-mode wave functions decay linear-exponentially, rather than as a power law. In Sec. VII A (VII B), we discuss only $T^{2}\left(T^{4}\right)$ compactification, where no topological 1-cycles (2-cycles) can shrink while keeping the volume of $T^{2}\left(T^{4}\right)$ finite. However, in more complicated geometries there are topological cycles that can do this. When a U(1) flux is introduced on such a topological 2-cycle, and if for some reason the 2-cycle shrinks, then symmetry breaking by the U(1) flux can be localized in extra dimensions, c.f. [29]. This contrasts with the situation in Secs. VII A and VII B, where the symmetry-breaking $\mathrm{U}(1)$ field strength is spread out homogeneously over the extra dimensions. If the symmetry-breaking $\mathrm{U}(1)$ flux is localized at a point in the extra dimensions, then fermion zero-mode wave functions decay linear-exponentially, with the exponent proportional to the distance from the symmetry-breaking source. Depending on the choice of parameters, the linear-exponential wave functions may or may not lead to as large a hierarchy as that which results from Gaussian wave functions. It would be interesting to study the effects on Yukawa-related observables of such a localized symmetry-breaking source, but this is beyond the scope of this paper.

To date, there has not been much investigation into what part of the moduli space of gauge-field backgrounds is more statistically weighted in flux compactification. It might be discovered that the statistic distribution is more weighted in regions that lead to Gaussian (or possibly linear-exponential) wave functions, so that large hierarchy among Yukawa couplings follows as a likely consequence. The other possibility is that environmental selection in favor of a light charged lepton (and/or quarks) enhances the statistical weight for such regions. We do not know which, if either, possibility is correct. However, for the landscape to account for the observed hierarchical patterns in the mass eigenvalues and mixing angles, we must assume one of these possibilities. As long as either of these possibilities is correct, then Gaussian landscapes should not be too terrible an approximation (or an effective description) of the landscape formulated by a super YangMills theory on higher-dimensional spacetime.

\section{D. $T^{3}$-fibered compactification and M(IIA)-theory dual}

In this paper we present numerical simulations of Gaussian landscapes involving only $D \leq 2$ extra dimen- 
sions. This is due to limited computational resources, not because there is any theoretical motivation to study landscapes involving $D \leq 2$. Indeed, more interesting would be to study Gaussian landscapes on $D=3$, since such a model would directly simulate (some fraction of) the landscape of Yukawa couplings in string theory. A Gaussian landscape on a three-dimensional manifold $B$ corresponds to heterotic string theory compactified on a sixdimensional manifold that is a $T^{3}$-fibration over $B$. To clarify, when one says that a $m$-dimensional manifold $X$ is a $T^{n}$-fibration over a manifold $B$, this means that the manifold $X$ locally resembles $T^{n} \times \mathbb{R}^{m-n}$ almost everywhere in $X$. For example, $T^{2}=T^{1} \times T^{1}$ and $T^{4}\left(x_{5,6,7,8}\right)=$ $T^{2}\left(x_{6,8}\right) \times T^{2}\left(x_{5,7}\right)$ in Secs. VII A and VII B are trivial examples of $T^{1}=S^{1}$ and $T^{2}$-fibered geometry. Here $B$ is called a base manifold, and $T^{n}$ a fiber.

Suppose that a six-dimensional manifold $X$ is a $T^{3}$-fibration over a three-dimensional manifold $B$. We choose a coordinate system locally, so that the $T^{3}$ direction is parametrized by $\left(x_{6}, x_{8}, x_{10}\right)$ and a local patch of $B$ by $\left(x_{5}, x_{7}, x_{9}\right)$. When the periods of $T^{3}$ in its three directions, $L_{6}, L_{8}$, and $L_{10}$, are small compared with the size of $B$ (that is, the cubic root of the volume of $B$ ), then a stable gaugefield configuration on $X$ is approximately described by fields $A_{6,8,10}$ that vary slowly on the coordinates $x_{5,7,9}$ of $B$. Fermion zero modes are localized at points on $B$ where all of $A_{6}\left(x_{5}, x_{7}, x_{9}\right), A_{8}\left(x_{5}, x_{7}, x_{9}\right)$, and $A_{10}\left(x_{5}, x_{7}, x_{9}\right)$ vanish. As long as $L_{6}, L_{8}$, and $L_{10}$ are all small, the zero-mode wave functions are approximately Gaussian [30]. Although $X$ is not globally the same as $T^{3} \times \mathbb{R}^{3}$ or $T^{3} \times T^{3}$, the local structure of $X$-its $T^{3}$-fibration-is sufficient to determine the approximately Gaussian zero-mode wave functions. Wave functions away from the Gaussian peak will depend on details of the global structure of $X$, but the wave functions are exponentially small in this region. Assuming a Gaussian profile with a fixed width for wave functions is surely a very crude approximation, but it might suffice as a zeroth order approximation. A Gaussian landscape on a $D=3$-dimensional manifold $B$ assumes that the moduli of stable gauge-field configurations are so rich that the center coordinates of various fields are scanned (almost) randomly and independently. The results of Sec. V provide a qualitative picture of how the distributions of observables depend on the base manifold $B$.

The most important parameter in a Gaussian landscape is the ratio of the Gaussian width to the size of the extra dimensions, $d / L$. For example, the overall hierarchy of Yukawa couplings is proportional to $(L / d)^{2}$ on a logarithmic scale. As we have seen in Sec. VII A, this ratio is proportional to $L_{5} / L_{6}=\operatorname{vol}\left(B=S^{1}\right) / \operatorname{vol}\left(T^{1}\right)$. This is generalized to

$$
\Delta \ln \lambda \propto\left[\frac{\operatorname{vol}(B)}{\operatorname{vol}\left(T^{3}\right)}\right]^{1 / 3} \sim \frac{1}{\alpha^{\prime}}\left[\operatorname{vol}(B) \times \operatorname{vol}\left(\hat{T}^{3}\right)\right]^{1 / 3} .
$$

As in Secs. VII A and VII B, the volume of the $T^{3}$ fiber has to be sufficiently smaller than that of the base manifold $B$ in order for hierarchy to be generated. It is an interesting question whether this property can be understood within string theory, not as a phenomenological requirement.

The heterotic string theory compactified on a $T^{3}$-fibered geometry corresponds to an 11-dimensional supergravity compactification on a $K 3$-fibered geometry (this is an intersecting D6-D6 system of the type IIA string theory) in the limit of small $\operatorname{vol}\left(T^{3}\right)$. Thus, Gaussian landscapes on three-folds for various values of $(d / L)$ are intended to simulate a class of vacua of string theory that interpolates between the heterotic theory and M-theory (ignoring stringy corrections). The expression in type IIA language (the last term) in (183) is known. Numerical simulation can tell us the value of $d / L$ that fits to the observed pattern of masses and mixings, which suggests the approximate value of (183) for the vacuum of our universe.

\section{E. $\boldsymbol{T}^{\mathbf{2}}$-Fibered compactification and F-theory dual}

The analysis of $D=2$ Gaussian landscapes in Sec. V may help one understand the landscape of Yukawa couplings of heterotic compactification on a $T^{2}$-fibered geometry. Let us now consider a six-dimensional manifold $X$ that is a $T^{2}$-fibration over a four-fold $B$. Let $\left(x_{9}, x_{10}\right)$ parametrize a local patch of the $T^{2}$-fibration and $\left(x_{5,6,7,8}\right)$ a local patch of $B$. Fermion zero modes are localized on a two-dimensional submanifold (called the matter curves) on $B$ so that the gauge fields $A_{9}\left(x_{5,6,7,8}\right)$ and $A_{10}\left(x_{5,6,7,8}\right)$ both vanish [31]. Furthermore, their wave functions around the matter curves are approximately Gaussian in the two transverse directions determined by $\nabla A_{9}$ and $\nabla A_{10}$. The existence of the two transverse directions in which zero-mode wave functions are Gaussian is quite similar to the $D=2$ Gaussian landscapes. One can determine the value of $d / L$ that fits the observed hierarchy of masses and mixing angles, which might then be used to infer $\operatorname{vol}(B)^{1 / 4} / \operatorname{vol}\left(T^{2}\right)^{1 / 2}$ for the $T^{2}$-fibered geometry. This class of vacua interpolates heterotic string theory and F(type IIB string)-theory.

There are also some features that are not captured by the $D=2$ Gaussian landscapes. Suppose that $A_{9}$ varies along $x_{7}$, and $A_{10}$ along $x_{8}$; at least it is possible to choose a coordinate system on $B$ locally so that this happens. Then a matter curve is along the $\left(x_{5}, x_{6}\right)$ directions. However the net chirality of a fermion depends on $F_{56}$, the gauge field along the matter curve. Therefore, zero-mode wave functions should have some $\left(x_{5}, x_{6}\right)$ dependence, but no such dependence is taken into account in the $D=2$ Gaussian landscapes. Moreover, the global geometry of matter curves may be complicated in $B$, and they can intersect one another, but the $D=2$ Gaussian landscapes ignore this as well. The behavior of zero-mode wave functions on a $T^{2}$-fibered geometry and the geometry of the intersection of matter curves must be studied further to determine 
whether the $D=2$ Gaussian landscapes can be useful in studying the landscapes of Yukawa couplings with $T^{2}$-fibered compactification.

\section{CONCLUSIONS}

This decade has seen the emergence of a major debate: to what extent is nature fundamentally uniquely prescribed, for example, by symmetries, vs to what extent nature results from the statistics of a huge landscape of vacua, modified by cosmological and environmental selection. For the standard model symmetries play a key role, but for physics beyond the standard model the question remains largely open. Unified gauge symmetries have striking achievements, for example, a simple interpretation of the quantum numbers of a generation and a precise numerical prediction for the ratios of the measured gauge couplings. For quark and lepton flavor, however, the picture offered by approximate flavor symmetries (AFS) is much less compelling, lacking both theoretical simplicity and significant successful predictions. Although the AFS description of flavor is apparently well suited to give an understanding of the hierarchical nature of charged fermion masses and the CKM mixing matrix, it comes with too much flexibility: with an appropriate choice of charges and symmetries any pattern of flavor can be generated.

In this paper we introduce Gaussian landscapes as some of the simplest landscapes in extra dimensions that can account for flavor. Particles are assumed to possess localized (Gaussian) zero-mode wave functions over some geometry of extra dimensions, and the Gaussian landscape consists of independently scanning the peak position of each of these wave functions. Small flavor-symmetrybreaking parameters are replaced by small overlap integrals of these wave functions on the extra dimensions. Localized zero-mode wave functions in extra dimensions are a natural expectation, and may have a more elegant realization than Higgs potentials for flavor symmetry breaking. We claim neither precise predictions nor do we present a compelling top-down theoretical model, rather we present the patterns of flavor that emerge from these simple toy landscapes, the features of these extradimensional landscapes that are relevant to the flavor problem, and how these landscapes might be realized within the context of string theory.

In the simplest Gaussian landscape describing the quark sector, where all quarks and the Higgs boson have a universal Gaussian wave function (but with independently scanning center coordinates) over a single extra dimension with geometry $S^{1}$, we find the three major characteristics of quark flavor: a hierarchical distribution of quark masses, pairing structure (the $W$-current approximately connects distinct pairs of quarks), and generation structure (the electroweak pairing connects the heaviest up-type quark to the heaviest down-type quark, and similarly for the middle and lightest quarks). The relevant probability dis- tributions are shown in Figs. 4 and 5 and result from inputting just two parameters: a universal constant $g_{\text {eff }}$ of order unity setting the scale for overlap integrals and a universal constant $d / L$ setting the width of Gaussian wave functions relative the size of the extra dimension.

The flavor structure of the quark sector can also be obtained from AFS using two free parameters. For example, by hypothesizing an approximate $\mathrm{U}(1)$ symmetry with a leading Yukawa coupling of order unity and others suppressed by various powers of a small symmetrybreaking parameter $\epsilon$. However there is a crucial difference. In the AFS case one must carefully choose the U(1) charges of each of the 15 fermions of the standard model. A huge variety of mass patterns could be accommodated by suitable charge choices. In the Gaussian landscape no such choices are made. Each of the 15 fermions is treated symmetrically. They differ only by the location of their Gaussian wave function, and these are scanned randomly over $S^{1}$. Thus the hierarchies arise purely from statistics; they cannot be changed as they do not involve any free parameters beyond $g_{\text {eff }}$ and $d / L$.

While the above accomplishments of the Gaussian landscape on $S^{1}$ are striking, there are certain features that are less than ideal. Although they are peaked, the probability distributions for flavor parameters are quite broad, as can be seen in Fig. 5. At half maximum, the deviation from the peak value is typically an order of magnitude. Thus the statistical nature of the landscape prevents us from making precise predictions. We find that this order of magnitude width is also typical of Gaussian landscapes in more than one dimension. The $S^{1}$ landscape makes no distinction between up and down sectors. This is a problem for explaining the observed large $t / b$ mass ratio, in particular, because on $S^{1}$ the distribution for the top and bottom masses is narrower than for the other generations and also because it is peaked near the maximum value. Thus even if there were a selection effect favoring a heavy top quark, the observed bottom mass would still be somewhat unlikely.

One possibility to resolve this shortcoming is to replace the effective coupling $g_{\text {eff }}$ with two parameters: one for the up sector that is about an order of magnitude larger than the one for the down sector. It would be interesting to find the landscape origin for such a " $\tan \beta$ " factor. Another possibility is that the shape of the $t / b$ mass distribution is a special feature of the $S^{1}$ landscape. We find that this is the case. In the $T^{2}$ landscape, where Gaussian wave functions are distributed at random over the surface of a (square) torus, the distribution is wider, as shown in Fig. 11. The same result holds for Gaussian landscapes on $S^{2}$ and $S^{3}$. A selection for a heavy quark, for example, for electroweak symmetry breaking, could then more easily account for the $t / b$ mass ratio. For any number of extra dimensions, we can analytically compute the Yukawa probability distribution near maximal values, and we find that it is suppressed 
as the dimension increases, strengthening this interpretation of the $t / b$ ratio.

Although flavor symmetries play no fundamental role in Gaussian landscapes, small overlap integrals may allow AFS to emerge in the low-energy theory. For example, if $q_{1}, q_{2}$, and $h$ have narrow Gaussian profiles with centers that are well separated from each other, then AFS that act on $q_{1}$ and $q_{2}$ will emerge at low energy. We have found that Yukawa matrices have the form expected from Abelian AFS, namely, $\lambda_{i j} \sim \epsilon_{i}^{\bar{q}} \epsilon_{j}^{q}$, if the Higgs width is not too wide and if the Yukawa coupling is not too small. As the separation between the Higgs boson and quarks approaches the scale of the extra dimension, effects associated with the periodicity of the wave functions destroys the AFS form of the Yukawa coupling. Nevertheless, the AFS approximation aids in understanding the numerical results, and we have used it to compute approximate analytic distributions for the AFS factors $\epsilon$, the quark mass eigenvalues, and the CKM mixing angles.

This strategy is particularly useful for understanding Gaussian landscapes on higher-dimensional geometries where numerical integration requires greater computational resources. Analytic results for the geometries $S^{1}$, $S^{2}, T^{2}=S^{1} \times S^{1}$, and $S^{3}$ have been obtained and the distributions are compared in Fig. 15. We find that the qualitative predictions of the Gaussian landscape on $S^{1}$ remain intact. One important feature is that, independent of the dimension $D$, the AFS distribution functions and flavor observables are found to be polynomial or logarithmic functions of $\ln \lambda_{i}$ or $\ln \left(\sin \theta_{i j}\right)$. By comparing numerical distributions of flavor parameters in Gaussian landscapes on $T^{2}$ and $S^{2}$, we have investigated the importance of the shape and curvature of the extra-dimensional manifold. We find some differences in the details, but the more striking are the similarities, as seen, for example, in Fig. 14.

Large leptonic mixing angles suggest that in the lowenergy theory no AFS emerges for the lepton doublets $l_{i}$, implying that the Gaussian widths for $l_{i}$ are comparable to the size of the extra dimension(s). Since the fermion mass hierarchies are smaller in the down and charged-lepton sectors than in the up sector, we economize on parameters and assume that the widths preserve a $\mathrm{SU}(5)$ symmetry, with $d_{5} \gg d_{10}, d_{H}$. The smallness of the neutrino masses suggests that there is no light right-handed neutrinos, such that the light neutrinos are Majorana and the origin of neutrino masses involves the breaking of $B-L$ symmetry. In the seesaw mechanism this implies Majorana masses for heavy right-handed neutrinos. In the Gaussian landscape we expect Gaussian profiles for both $\bar{\nu}_{R}$ and the $B-L$ breaking fields. The center of the $B-L$ breaking is not expected to be correlated with the center of $\mathrm{SU}(2) \times$ $\mathrm{U}(1)$ breaking, leading to a very different statistical character for the Majorana and Dirac mass matrices of neutrinos.
We find that the physics of neutrino masses and mixings is quite different depending on whether the profile for $\bar{\nu}_{R}$ is narrow or wide. In the case that it is narrow, localization leads to significant hierarchies in the eigenvalues of both the Majorana and Dirac matrices. In theories based on AFS these hierarchies cancel in the light neutrino mass matrix, but in the Gaussian landscape these hierarchies add. The result is that $m_{2} / m_{3}$ is typically too small to agree with observation, unless perhaps if strong selection effects are important. For wide $\bar{\nu}_{R}$ profiles, the neutrino Yukawa and Majorana matrices take on a democratic form, and again have hierarchical eigenvalues. If the profiles are sufficiently wide, $m_{2} / m_{3}$ is always less than the observed value, as shown in Fig. 19. Similar difficulties arise with the lepton mixing angle $\theta_{23}$. Although a large width for the lepton doublets leads to large 23 rotations to diagonalize the charged and neutral lepton mass matrices, these large angles cancel so that the physical mixing angle $\theta_{23}$ is typically small, as shown in the numerical simulation of Fig. 21.

These difficulties, that $m_{2} / m_{3}$ and $\theta_{23}$ are typically much smaller than the values observed, are both solved by a remarkably simple observation. In order to account for $C P$ violation in the quark sector a complex phase must be introduced to the Gaussian profile. Using the simple profile of (88), we find the following results for the Gaussian landscape on $S^{1}$ :

(i) Previous results for distributions of charged fermion masses are preserved, for example, Fig. 16 is essentially unchanged for $C P$-violating Gaussian landscapes.

(ii) The generation structure of the quark sector is preserved (Fig. 24).

(iii) The CKM phase is of order unity (Fig. 25).

(iv) Large leptonic mixing angles $\theta_{12}$ and $\theta_{23}$ are obtained (Fig. 23).

(v) The large observed value for the neutrino mass ratio $m_{2} / m_{3}$ is not atypical [Fig. 20(h)].

The distributions of flavor observables on Gaussian landscapes are typically broad. However, given the experimental measurement of some subset of flavor observables, the conditional distributions for the remaining observables in our universe changes significantly. We find that even quite loose cuts on the measured neutrino parameters are sufficient to considerably sharpen the predictions for the leptonic mixing angle, $\theta_{13}$, and the neutrinoless double beta parameter, $m_{\beta \beta}$, within a particular Gaussian landscape; see Fig. 26 and (102), respectively. Furthermore, these cuts lead to a prediction of large $C P$ violation in neutrino oscillation as shown in the last row of Fig. 25 and in the scatter plots of Fig. 26.

When a supersymmetric gauge theory is compactified on an internal manifold, quarks, leptons, and the Higgs boson may originate from gauginos and the components of gauge fields with polarization along the internal manifold. Their 
Yukawa interactions originate from the gauge interactions of the gauginos, while the Yukawa coupling constants are calculated by overlap integration of zero-mode wave functions over the extra-dimensional space. Wave functions become Gaussian when the extra-dimensional manifold is a torus fibration, and the fiber is small relative to the base. Thus, Gaussian landscapes fit very well into this framework - compactification of a supersymmetric gauge theory on a torus-fibered manifold.

The ratio $d / L$, which controls the hierarchy, roughly reflects the ratio of the torus fiber size to the base size in heterotic theory language. We now know the value of $d / L$ that is good for quark and lepton phenomenology, but it remains an open question why such a value has been selected from all the possibilities in the landscape (or if it represents a typical subset of the landscape). Indeed, there is no theoretical motivation to consider torus-fibered compactification over the myriad of other geometries for compactification. We also assumed in the Gaussian landscape that the center coordinates of all Gaussian wave functions scan randomly and independently over the base space. This assumption is not totally without motivation, but it would be nice if the validity of this assumption were studied in string theory.

Simple Gaussian landscapes, motivated by supersymmetric gauge theories compactified on a torus-fibered manifold, can account for the broad pattern of quark and lepton masses and mixings in terms of just five parameters. While the probability distributions for the measured parameters are broad, predictions from a given landscape for future measurements in neutrino physics are more precise.

\section{ACKNOWLEDGMENTS}

This work was supported in part by NSF Grant No. PHY04-57315 (L. J. H.), the U.S. DOE under Contract No. DEAC03-76SF00098 (L. J.H.) and No. DE-FG0392ER40701 (M.P.S., T.W.), and the Gordon and Betty Moore Foundation (T.W.). We thank Aspen Center for Physics (L. J.H., T.W.), UC Berkeley CTP (T.W.), and MIT CTP (T. W.) for hospitality.

\section{APPENDIX: APPROXIMATE PROBABILITY DISTRIBUTION FUNCTIONS}

We here collect the probability distribution functions calculated using the AFS approximation, i.e. using (36) and (38)-(40), for the Gaussian landscape on $S^{1}$. First we list the distribution functions of the Yukawa eigenvalues that follow from (33) and the approximation (36):

$$
\begin{aligned}
& \frac{d P\left(z_{3}\right)}{d z_{3}}= \begin{cases}\frac{9}{4}\left(\pi-8 \sqrt{z_{3}}+(4+\pi) z_{3}-\frac{8}{3} z_{3}^{3 / 2}+\frac{\pi}{8} z_{3}^{2}\right) & \text { for } 0 \leq z_{3} \leq 1, \\
-\frac{3}{16}\left[32-76 \sqrt{z_{3}-1}+48 z_{3}-26 z_{3} \sqrt{z_{3}-1}\right. & \\
-3\left(8+8 z_{3}+z_{3}^{2}\right) \operatorname{arccot}\left(\sqrt{z_{3}}-1\right) & \text { for } 1<z_{3} \leq 2 . \\
\left.-3\left(8+8 z_{3}+z_{3}^{2}\right) \arctan \left(\sqrt{z_{3}}-1\right)\right] & \text { for } 0 \leq z_{2} \leq 1,\end{cases} \\
& \frac{d P\left(z_{2}\right)}{d z_{2}}= \begin{cases}\frac{3}{8} z_{2}\left(24-32 \sqrt{z_{2}}+3 \pi z_{2}\right) & \text { for } 1<z_{2} \leq 2 . \\
\frac{3}{2}\left(4-2 \sqrt{z_{2}}-1-6 z_{2}+5 z_{2} \sqrt{z_{2}-1}\right) & \text { for } 0 \leq z_{1} \leq 1, \\
-\frac{9}{4} z_{2}^{2}\left[\arctan \left(\sqrt{z_{2}}-1\right)-\operatorname{arccot}\left(\sqrt{z_{2}-1}\right)\right] & \text { for } 1<z_{1} \leq 2 .\end{cases} \\
& \frac{d P\left(z_{1}\right)}{d z_{1}}=\left\{\begin{array}{l}
\frac{9 \pi}{32} z_{1}^{2} \\
\frac{9}{16}\left\{2\left(2-z_{1}\right) \sqrt{z_{1}-1}\right. \\
\left.-z_{1}^{2}\left[\arctan \left(\sqrt{z_{1}}-1\right)-\operatorname{arccot}\left(\sqrt{z_{1}-1}\right)\right]\right\}
\end{array}\right.
\end{aligned}
$$

Here $\quad z_{i} \equiv \ln \left(\lambda_{i} / \lambda_{\max }\right) / \Delta \ln \epsilon \quad$ where $\quad \lambda_{\max }=$ $\left(4 L^{2} / 9 \pi d^{2}\right)^{1 / 4} g_{\text {eff }}$ and $\Delta \ln \epsilon=-(L / d)^{2} / 12$. We also note the mean values of these distributions:

$$
\left\langle z_{3}\right\rangle=0.2, \quad\left\langle z_{2}\right\rangle=0.6, \quad\left\langle z_{1}\right\rangle=1.2 .
$$

Meanwhile, the distribution functions of the CKM mixing angles follow from (33) and the set of approximations (38) - (40). They are given by

$$
\begin{aligned}
\frac{d P\left(t_{12}\right)}{d t_{12}}=6\left(1-\sqrt{t_{12}}\right)^{3}\left(1+2 \sqrt{t_{12}}\right) d t_{12}, \\
\frac{d P\left(t_{23}\right)}{d t_{23}}=\frac{3}{2}\left[-2 \sqrt{1-t_{23}}+\operatorname{arccosh}\left(\frac{2}{t_{23}}-1\right)\right] \\
\times\left[2 \sqrt{1-t_{23}}\left(1+2 t_{23}\right)\right. \\
\left.-3 t_{23} \operatorname{arccosh}\left(\frac{2}{t_{23}}-1\right)\right],
\end{aligned}
$$




$$
\begin{aligned}
\frac{d P\left(t_{13}\right)}{d t_{13}}= & 6\left(\sqrt{1-t_{13}}+\sqrt{t_{13}}-1\right)\left(1-\sqrt{t_{13}}\right) \\
& \times\left[2 \sqrt{1-t_{13}}-1-\sqrt{t_{13}}+2 t_{13}\right. \\
& \left.+2 \sqrt{t_{13}\left(1-t_{13}\right)}\right],
\end{aligned}
$$

where each applies over the full range $0 \leq t_{i j} \leq 1$, with $t_{i j} \equiv \ln \left|\sin \theta_{i j}\right| / \Delta \ln \epsilon$. The mean values of these distributions are

$$
\left\langle t_{12}\right\rangle=0.17, \quad\left\langle t_{23}\right\rangle=0.10, \quad\left\langle t_{13}\right\rangle=0.35 .
$$

[1] C. D. Froggatt and H. B. Nielsen, Nucl. Phys. B147, 277 (1979).

[2] S. Perlmutter et al. (Supernova Cosmology Project Collaboration), Nature (London) 391, 51 (1998); B.P. Schmidt et al. (Supernova Search Team Collaboration), Astrophys. J. 507, 46 (1998); A. G. Riess et al. (Supernova Search Team Collaboration), Astron. J. 116, 1009 (1998).

[3] S. Weinberg, Phys. Rev. Lett. 59, 2607 (1987); H. Martel, P. R. Shapiro, and S. Weinberg, Astrophys. J. 492, 29 (1998).

[4] R. Bousso and J. Polchinski, J. High Energy Phys. 06 (2000) 006.

[5] E. A. Mirabelli and M. Schmaltz, Phys. Rev. D 61, 113011 (2000).

[6] C. J. Hogan, Phys. Rev. D 74, 123514 (2006), and references therein.

[7] B. Feldstein, L. J. Hall, and T. Watari, Phys. Rev. D 74, 095011 (2006).

[8] L. J. Hall, H. Murayama, and N. Weiner, Phys. Rev. Lett. 84, 2572 (2000).

[9] J. F. Donoghue, K. Dutta, and A. Ross, Phys. Rev. D 73, 113002 (2006).

[10] N. Arkani-Hamed and M. Schmaltz, Phys. Rev. D 61, 033005 (2000).

[11] L. J. Hall, M. P. Salem, and T. Watari, arXiv:0707.3444.

[12] N. Haba and H. Murayama, Phys. Rev. D 63, 053010 (2001).

[13] J. F. Donoghue, Phys. Rev. D 69, 106012 (2004); 69, 129901(E) (2004).

[14] W. M. Yao et al. (Particle Data Group), J. Phys. G 33, 1 (2006).

[15] A. de Gouvea and H. Murayama, Phys. Lett. B 573, 94 (2003).
[16] N. Arkani-Hamed, S. Dimopoulos, and S. Kachru, arXiv:hep-th/0501082.

[17] A. Hebecker and J. March-Russell, Phys. Lett. B 541, 338 (2002).

[18] H. Harari, H. Haut, and J. Weyers, Phys. Lett. 78B, 459 (1978); Y. Koide, Phys. Rev. D 28, 252 (1983); 39, 1391 (1989).

[19] R. Tatar and T. Watari, Nucl. Phys. B747, 212 (2006).

[20] See, for example, Ref. [12] of [10].

[21] M. Hamanaka and H. Kajiura, Phys. Lett. B 551, 360 (2003).

[22] D. Cremades, L.E. Ibanez, and F. Marchesano, J. High Energy Phys. 05 (2004) 079.

[23] G. Aldazabal, L. E. Ibanez, F. Quevedo, and A. M. Uranga, J. High Energy Phys. 08 (2000) 002.

[24] G. 't Hooft (unpublished).

[25] M. F. Atiyah and I. M. Singer, Ann. Math. 87, 484 (1968); M. Atiyah, R. Bott, and V.K. Patodi, Inventiones Mathematicae 19, 279 (1973).

[26] C. Taubes, J. Diff. Geom. 19, 517 (1984).

[27] E. Corrigan and P. Goddard, Ann. Phys. (N.Y.) 154, 253 (1984).

[28] N. S. Craigie, P. Goddard, and W. Nahm, in Proceedings of Monopoles in Quantum Field Theory, Trieste, Italy, 1981, (World Scientific, Singapore, 1982); Self-Dual Monopoles and Calorons, in Lecture Notes in Physics Vol. 201 (Springer, New York, 1984); H. Schenk, Commun. Math. Phys. 116, 177 (1988); P. J. Braam and P. van Baal, Commun. Math. Phys. 122, 267 (1989).

[29] L. J. Hall and Y. Nomura, Phys. Rev. D 64, 055003 (2001).

[30] B. Acharya and E. Witten, arXiv:hep-th/0109152.

[31] R. Friedman, J. Morgan, and E. Witten, Commun. Math. Phys. 187, 679 (1997). 